\title{
Gradings of positive rank on simple Lie algebras
}

\author{
Mark Reeder* \\ reederma@bc.edu \\ Jiu-Kang $\mathrm{Yu}^{\dagger}$ \\ jyu@math.purdue.edu
}

\author{
Paul Levy \\ p.d.levy@lancaster.ac.uk \\ Benedict H. Gross $\ddagger$ \\ gross@math.harvard.edu
}

June 22, 2012

\section{Contents}

1 Introduction

2 Kac coordinates $\quad 6$

2.1 Based automorphisms and affine root systems . . . . . . . . . . . . . 6

2.2 Torsion points, Kac coordinates and the normalization algorithm . . . . . . . . . 9

$2.3 \mu_{m}$-actions on Lie algebras $\ldots \ldots \ldots \ldots$

2.4 Principal $\mu_{m}$-actions $\ldots \ldots \ldots \ldots \ldots \ldots \ldots$

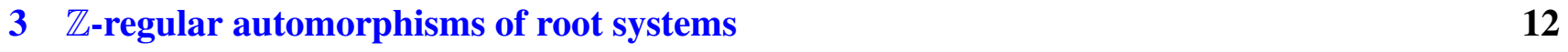

4 Positive rank gradings $\quad 16$

4.1 A canonical Cartan subalgebra . . . . . . . . . . . . . . . . 16

4.2 A relation between $\operatorname{Aut}(\mathfrak{g})$ and $\operatorname{Aut}(R) \ldots \ldots \ldots \ldots \ldots$

4.3 Inner automorphisms . . . . . . . . . . . . . . . . 17

5 Principal and stable gradings $\quad 20$

* Supported by NSF grants DMS-0801177 and DMS-0854909

${ }^{\dagger}$ Supported by NSF grant DMS-0854909

${ }^{\ddagger}$ Supported by NSF grant DMS-0901102 
5.1 Principal gradings . . . . . . . . . . . . . . . . . . . 20

5.2 Conjugacy results . . . . . . . . . . . . . . . . . . 21

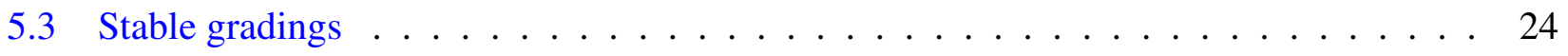

6 Affine-pinned automorphisms 25

7 Little Weyl groups $\quad 28$

7.1 Upper bounds on the little Weyl group . . . . . . . . . . . . . . . . . . . . . . 28

7.2 Little Weyl groups for inner gradings . . . . . . . . . . . . . . . . . . . . . . 29

7.3 Stable isotropy groups . . . . . . . . . . . . . . . . . 32

7.4 Stable orbits and elliptic curves . . . . . . . . . . . . . . . 33

8 Classification of stable gradings $\quad 34$

8.1 Stable gradings of exceptional Lie algebras $\ldots \ldots \ldots$. . . . . . . . . . . 35

8.2 Stable gradings of classical Lie algebras . . . . . . . . . . . . . . . . 37

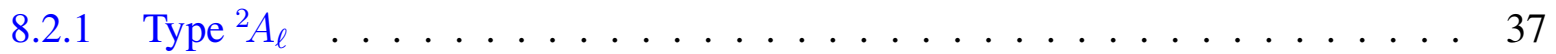

8.2.2 Types $B_{n}, C_{n} \ldots \ldots \ldots \ldots \ldots \ldots$

8.2.3 Types $D_{n}$ and ${ }^{2} D_{n} \quad(n \geq 4) \ldots \ldots \ldots \ldots$

8.3 Distinguished nilpotent elements and stable gradings . . . . . . . . . . . . 41

9 Positive rank gradings for type $E_{6,7,8}$ (inner case) 43

9.1 A preliminary list of Kac coordinates for positive rank gradings of inner type . . . . . 43

9.2 Tables of positive rank gradings for $E_{6}, E_{7}$ and $E_{8} \ldots \ldots \ldots$. . . . . . . 48

10 Little Weyl groups for inner type $E$ and Kostant sections $\quad 54$

10.1 The Levi subgroup $L_{\theta} \ldots \ldots \ldots \ldots$. . . . . . . . . . . . . . . . . 54

10.1 .1 Example: $E_{6}$ no. $4_{b} \ldots \ldots \ldots \ldots \ldots$

10.1 .2 Example: $E_{7}$ no. $5_{d} \ldots \ldots \ldots \ldots \ldots$

10.1 .3 Example: $E_{7}$ no. $4_{d} \ldots \ldots \ldots \ldots \ldots \ldots$

10.1.4 Example: $E_{7}$ no. $4_{e} \ldots \ldots \ldots \ldots \ldots \ldots$

10.1.5 Example: $E_{8}$ no. $4_{e} \ldots \ldots \ldots \ldots$ 
10.2 A remark on saturation . . . . . . . . . . . . . . . . . . 59

10.3 Kostant sections and the Levi subgroup $L_{\theta} \ldots \ldots \ldots \ldots$. . . . . . . . . . . . . . 59

11 Outer gradings of positive rank in type $E_{6} \quad 60$

11.1 Root systems of type $E_{7}$ and ${ }^{2} E_{6} \ldots \ldots \ldots \ldots$. . . . . . . . . . 60

11.2 Lie algebras of type $E_{7}$ and ${ }^{2} E_{6} \ldots \ldots \ldots \ldots \ldots$

11.3 Positive rank gradings on $E_{6}$ (outer case) $\ldots \ldots \ldots$. . . . . . . . . . . . 64

11.4 Little Weyl groups for ${ }^{2} E_{6} \ldots \ldots \ldots$. . . . . . . . . . . . . . 66

11.5 Standard subalgebras and Kostant sections . . . . . . . . . . . . . . . 67

\section{Introduction}

Let $\mathfrak{g}$ be the Lie algebra of a connected simple algebraic group $G$ of adjoint type over an algebraically closed field $k$. A grading on $\mathfrak{g}$ is a decomposition

$$
\mathfrak{g}=\bigoplus_{i \in \mathbb{Z} / m} \mathfrak{g}_{i}
$$

where $m$ is an integer $\geq 0$ and $\left[\mathfrak{g}_{i}, \mathfrak{g}_{j}\right] \subset \mathfrak{g}_{i+j}$ for all $i, j$. The summand $\mathfrak{g}_{0}$ is a Lie subalgebra of $\mathfrak{g}$ and we let $G_{0}$ denote the corresponding connected subgroup of $G$. The adjoint action of $G$ on $\mathfrak{g}$ restricts to an action of $G_{0}$ on each summand $\mathfrak{g}_{i}$. We are interested in the invariant theory of this action, for which there is no loss of generality if we assume that $i=1$.

If $m=1$ this is the invariant theory of the adjoint representation, first developed by Chevalley, who showed that the restriction $k[\mathfrak{g}]^{G} \rightarrow k[\mathfrak{t}]^{W}$ of $G$-invariant polynomials on $\mathfrak{g}$ to polynomials on a Cartan subalgebra $\mathfrak{t}$ invariant under the Weyl group $W$ is an isomorphism. This and other aspects of Chevalley's theory were generalized to the case $m=2$ by Kostant and Rallis [16]. Soon after, Vinberg [35] showed that for any $m \geq 0$ the invariant theory of the $G_{0}$-action on $\mathfrak{g}_{1}$ has similar parallels with the adjoint representation of $G$ on $\mathfrak{g}$. Vinberg worked over $\mathbb{C}$, but in [19], Vinberg's theory was extended to fields of good odd positive characteristic not dividing $m$.

Some highlights of Vinberg theory are as follows. A Cartan subspace is a linear subspace $\mathfrak{c} \subset \mathfrak{g}_{1}$ which is abelian as a Lie algebra, consists of semisimple elements, and is maximal with these two properties. All Cartan subspaces are conjugate under $G_{0}$. Hence the dimension of $\mathfrak{c}$ is an invariant of the grading, called the rank, which we denote in this introduction by $r$. The little Weyl group is the subgroup $W_{\mathfrak{c}}$ of $\mathrm{GL}(\mathfrak{c})$ arising from the action of the normalizer of $\mathfrak{c}$ in $G_{0}$. The group $W_{\mathfrak{c}}$ is finite and is generated by semisimple transformations of $\mathfrak{c}$ fixing a hyperplane and we have an isomorphism of invariant polynomial rings

$$
k\left[\mathfrak{g}_{1}\right]^{G_{0}} \stackrel{\sim}{\sim} k[\mathfrak{c}]^{W_{\mathfrak{c}}}
$$

given by restriction. Finally $k\left[\mathfrak{g}_{1}\right]^{G_{0}} \simeq k\left[f_{1}, \ldots, f_{r}\right]$ is a polynomial algebra generated by $r$ algebraically independent polynomials $f_{1}, \ldots, f_{r}$ whose degrees $d_{1}, \ldots, d_{r}$ are determined by the grading. In particular the product of these degrees is the order of $W_{\mathfrak{c}}$. 
We have a dichotomy: either the rank $r=0$, in which case $\mathfrak{g}_{1}$ consists entirely of nilpotent elements of $\mathfrak{g}$, or $r>0$, in which case $m>0$ and $\mathfrak{g}_{1}$ contains semisimple elements of $\mathfrak{g}$. A basic problem is to classify all gradings of rank $r>0$ and to compute the little Weyl groups $W_{\mathfrak{c}}$ in each case. Another open question is Popov's conjecture: $\mathfrak{g}_{1}$ should contain a Kostant section: an affine subspace $\mathfrak{v}$ of $\mathfrak{g}_{1}$ with $\operatorname{dim} \mathfrak{v}=r$, such that the restriction map $k\left[\mathfrak{g}_{1}\right]^{G_{0}} \longrightarrow k[\mathfrak{v}]$ is an isomorphism.

The classification of positive-rank gradings and their little Weyl groups, along with verification of Popov's conjecture was given in [19] and [20] for gradings of Lie algebras of classical type and those of types $G_{2}$ and $F_{4}$. In this paper we complete this work by proving analogous results for types $E_{6}, E_{7}$ and $E_{8}$, using new methods which apply to the Lie algebras of general simple algebraic groups $G$.

The main idea is to compute Kac coordinates of lifts of automorphisms of the root system $R$ of $\mathfrak{g}$, as we shall now explain. Choosing a base in $R$ and a pinning in $\mathfrak{g}$ (defined in section 2.3), we may write the automorphism groups $\operatorname{Aut}(R)$ and $\operatorname{Aut}(\mathfrak{g})$ as semidirect products:

$$
\operatorname{Aut}(R)=W \rtimes \Theta, \quad \operatorname{Aut}(\mathfrak{g})=G \rtimes \Theta,
$$

where $W$ is the Weyl group of $R$ and $\Theta$, the symmetry group of the Dynkin graph $D(R)$ of $R$, is identified with the group of automorphisms of $\mathfrak{g}$ fixing the chosen pinning. To each $\vartheta \in \Theta$ one can associate an affine root system $\Psi=\Psi(R, \vartheta)$ consisting of affine functions on an affine space $\mathcal{A}$ of dimension equal to the number of $\vartheta$-orbits on the nodes of the diagram $D(R)$. Kac' original construction of $\Psi$ uses infinite dimensional Lie algebras and works over $\mathbb{C}$; our approach constructs $\Psi$ directly from the pair $(R, \vartheta)$ and works over any algebraically closed field in which the order $e$ of $\vartheta$ is nonzero. The choice of pinning on $\mathfrak{g}$ determines a rational structure on $\mathcal{A}$ and a basepoint $x_{0} \in \mathcal{A}$. Following an idea of Serre [26], we associate to each rational point $x \in \mathcal{A}_{\mathbb{Q}}$ an embedding $\varrho_{x}: \boldsymbol{\mu}_{m} \hookrightarrow G$ of group schemes over $k$, where $m$ is the denominator of $x$. If $m$ is nonzero in $k$ and we choose a root of unity $\zeta \in k^{\times}$of order $m$, then $x$ determines an actual automorphism $\theta_{x} \in G \vartheta$ of order $m$. If $x$ lies in the closure $\bar{C}$ of the fundamental alcove of $\mathcal{A}$ then the affine coordinates of $x$ are those defined by $\mathrm{Kac}$ (when $k=\mathbb{C}$ and $\zeta=e^{2 \pi i / m}$ ); we call these normalized Kac coordinates, since we also consider points $x$ outside $\bar{C}$ having some affine coordinates negative. Any $x \in \mathcal{A}_{\mathbb{Q}}$ can be moved into $\bar{C}$ via operations of the affine Weyl group $W(\Psi)$, and this can be done effectively, using a simple algorithm. See also [20], which gives a different way of extending Kac coordinates to positive characteristic.

The half-sum of the positive co-roots is a vector $\check{\rho}$ belonging to the translation subgroup of $\mathcal{A}$. In the principal segment $\left[x_{0}, x_{0}+\check{\rho}\right] \subset \mathcal{A}$ we are especially interested in the points

$$
x_{m}:=x_{0}+\frac{1}{m} \check{\rho} \in \mathcal{A}_{\mathbb{Q}},
$$

where $m$ is the order of an elliptic $\mathbb{Z}$-regular automorphism $\sigma \in \operatorname{Aut}(R)$. Here $\sigma$ is elliptic if $\sigma$ has no nonzero fixed-points in the reflection representation, and we say $\sigma$ is $\mathbb{Z}$-regular if the group generated by $\sigma$ acts freely on $R$. (This is almost equivalent to Springer's notion of regularity, and for our purposes it is the correct one. See section 3.)

Now assume that the characteristic of $k$ is not a torsion prime for $\mathfrak{g}$.

Choose a Cartan subalgebra $\mathfrak{t}$ of $\mathfrak{g}$, let $T$ be the maximal torus of $G$ centralizing $\mathfrak{t}$ with normalizer $N$ in $G$ and let $\operatorname{Aut}(\mathfrak{g}, \mathfrak{t})$ be the subgroup of $\operatorname{Aut}(\mathfrak{g})$ preserving $\mathfrak{t}$. The groups $\operatorname{Aut}(R)$ and $\operatorname{Aut}(\mathfrak{g}, \mathfrak{t}) / T$ are isomorphic and we may canonically identify $W$-conjugacy classes in $\operatorname{Aut}(R)$ with $N / T$-conjugacy 
classes in $\operatorname{Aut}(\mathfrak{g}, \mathfrak{t}) / T$. Let $\sigma \in \operatorname{Aut}(R)$ be an elliptic $\mathbb{Z}$-regular automorphism whose order $m$ is nonzero in $k$. Write $\sigma=w \cdot \vartheta$ with $w \in W$ and $\vartheta \in \Theta$. Then there is a unique $G$-conjugacy class $C_{\sigma} \subset G \vartheta$ such that $C_{\sigma} \cap \operatorname{Aut}(\mathfrak{g}, \mathfrak{t})$ projects to the class of $\sigma$ in $\operatorname{Aut}(R)$. Using results of Panyushev in [23], we show that $C_{\sigma}$ contains the automorphism $\theta_{x_{m}}$, where $x_{m}$ is the point on the principal segment defined above. The (un-normalized) Kac coordinates of $x_{m}$ are all $=1$ except one coordinate is $1+\left(m-h_{\vartheta}\right) / e$, where $h_{\vartheta}$ is the twisted Coxeter number of $(R, \vartheta)$. Translating by the affine Weyl group we obtain the normalized Kac coordinates of the class $C_{\sigma} \subset G \vartheta$. The automorphisms in $C_{\sigma}$ have positive rank equal to the multiplicity of the cyclotomic polynomial $\Phi_{m}$ in the characteristic polynomial of $\sigma$. They are exactly the semisimple automorphisms of $\mathfrak{g}$ for which $G_{0}$ has stable orbits in $\mathfrak{g}_{1}$, in the sense of Geometric Invariant Theory.

Every $G$-conjugacy class of positive-rank automorphisms $\theta \in \operatorname{Aut}(\mathfrak{g})$ whose order is nonzero in $k$ contains a lift of a $W$-conjugacy class in $\operatorname{Aut}(R)$. For any particular group $G$ we can tabulate the Kac coordinates of such lifts; these are exactly the Kac coordinates of positive rank gradings. For this purpose it is enough to consider only the lifts of certain classes in $\operatorname{Aut}(R)$, almost all of which are elliptic and $\mathbb{Z}$-regular in $\operatorname{Aut}\left(R^{\prime}\right)$ for some root subsystem of $R$, whose Kac coordinates are easily found, as above.

These tables are only preliminary because they contain some Kac diagrams more than once, reflecting the fact that a given class in $\operatorname{Aut}(\mathfrak{g})$ may contain lifts of several classes of $\sigma \in \operatorname{Aut}(R)$. However, each class in $\operatorname{Aut}(\mathfrak{g})$ has a "best" $\sigma$ whose properties tell us about other aspects of the grading, for example the little Weyl group $W(\mathfrak{c})$. Our final tables for $E_{6}, E_{7}$ and $E_{8}$ list each positive rank Kac diagram once and contain this additional data.

Besides its contributions to Vinberg theory per se, this paper was motivated by connections between Vinberg theory and the structure and representation theory of a reductive group $\mathbf{G}$ over a $p$-adic field $F$. The base field $k$ above is then the residue field of a maximal unramified extension $L$ of $F$. We assume $\mathbf{G}$ splits over a tame extension $E$ of $L$. Then the Galois group $\operatorname{Gal}(E / L)$ is cyclic and acts on the root datum of $\mathbf{G}$ via a pinned automorphism $\vartheta$. The grading corresponds to a point $x$ in the BruhatTits building of $\mathbf{G}(L)$, the group $G_{0}$ turns out to be the reductive quotient of the parahoric subgroup $\mathbf{G}(L)_{x}$ fixing $x$, and the summands $\mathfrak{g}_{i}$ are quotients in the Moy-Prasad filtration of $\mathbf{G}(L)_{x}$. As we will show elsewhere, the classification of positive rank gradings leads to a classification of non-degenerate $K$-types, a long outstanding problem in the representation theory of $\mathbf{G}(F)$, and stable $G_{0}$-orbits in the dual of $\mathfrak{g}_{1}$ give rise to supercuspidal representations of $\mathbf{G}(F)$ attached to elliptic $\mathbb{Z}$-regular elements of the Weyl group. These generalize the "simple supercuspidal representations" constructed in [11], which correspond to the Coxeter element.

After the first version of this paper was written, we learned from A. Elashvili that 25 years ago he, D. Panyushev and E. Vinberg had also calculated, by completely different methods, all the positive rank gradings and little Weyl groups in types $E_{6,7,8}$ (for $k=\mathbb{C}$ ) but they had never published their results. We thank them for comparing their tables with ours. For other aspects of positive-rank gradings on exceptional Lie algebras, see [9]. 


\section{Kac coordinates}

Kac [12, chap. 8] showed how conjugacy classes of torsion automorphisms of simple Lie algebras $\mathfrak{g}$ (over $\mathbb{C}$ ) can be parametrized by certain labelled affine Dynkin diagrams, called Kac coordinates. If we choose a root of unity $\zeta \in \mathbb{C}^{\times}$of order $m$, then any automorphism $\theta \in \mathfrak{g}$ of order $m$ gives a grading $\mathfrak{g}=\bigoplus_{i \in \mathbb{Z} / m} \mathfrak{g}_{i}$, where $\mathfrak{g}_{i}$ is the $\zeta^{i}$-eigenspace of $\theta$. This grading depends on the choice of $\zeta$ and if we replace $\mathbb{C}$ by another ground field $k$, we are forced to assume that $m$ is invertible in $k$. As in [19], this assumption will be required for our classification of positive-rank automorphisms.

However, at the level of classifying all torsion automorphisms, Serre has remarked (see [26]) that, at least in the inner case, one can avoid the choice of $\zeta$ and restrictions on $k$ by replacing an automorphism $\theta$ of order $m$ with an embedding $\boldsymbol{\mu}_{m} \hookrightarrow \operatorname{Aut}(\mathfrak{g})^{\circ}$ of group schemes over $k$, where $\boldsymbol{\mu}_{m}$ is the group scheme of $m^{\text {th }}$ roots of unity.

In this section we give an elementary treatment of Kac coordinates in Serre's more general setting, and we extend his approach to embeddings $\boldsymbol{\mu}_{m} \hookrightarrow \operatorname{Aut}(\mathfrak{g})$. In the outer case, where the image of $\boldsymbol{\mu}_{m}$ does not lie in $\operatorname{Aut}(\mathfrak{g})^{\circ}$, we still find it necessary to assume the characteristic $p$ of $k$ does not divide the order of the projection of $\boldsymbol{\mu}_{m}$ to the component group of $\operatorname{Aut}(\mathfrak{g})$. Our approach differs from [12] in that we avoid infinite dimensional Lie algebras (cf. [24]).

We then discuss a family of examples, the principal embeddings of $\boldsymbol{\mu}_{m}$, which play an important role in gradings of positive rank.

\subsection{Based automorphisms and affine root systems}

For background on finite and affine root systems see [6] and [21]. Let $R$ be an irreducible reduced finite root system spanning a real vector space $V$. The automorphism group of $R$ is the subgroup of GL( $V)$ preserving $R$ :

$$
\operatorname{Aut}(R)=\{\sigma \in \mathrm{GL}(V): \sigma(R)=R\} .
$$

We say an automorphism $\sigma \in \operatorname{Aut}(R)$ is based if $\sigma$ preserves a base of $R$. If we choose a base $\Delta$ of $R$ then we have a splitting

$$
\operatorname{Aut}(R)=W \rtimes \Theta,
$$

where $W$ is the Weyl group of $R$ and $\Theta=\{\sigma \in \operatorname{Aut}(R): \sigma(\Delta)=(\Delta)\}$. Since $R$ is irreducible, the group $\Theta$ is isomorphic to a symmetric group $S_{n}$ for $n=1,2$ or 3 .

In this section we will associate to any based automorphism $\vartheta \in \operatorname{Aut}(R)$ an affine root system $\Psi(R, \vartheta)$ whose isomorphism class will depend only on the order $e$ of $\vartheta$.

We first establish more notation to be used throughout the paper. Let $X=\mathbb{Z} R$ be the lattice in $V$ spanned by $R$ and let $\check{X}=\operatorname{Hom}(X, \mathbb{Z})$ be the dual lattice. We denote the canonical pairing between $X$ and $\check{X}$ by $\langle\lambda, \check{\omega}\rangle$, for $\lambda \in X$ and $\check{\omega} \in \check{X}$.

Fix a base $\Delta=\left\{\alpha_{1}, \ldots, \alpha_{\ell}\right\}$ of $R$, where $\ell$ is the rank of $R$, and let $\check{R} \subset \check{X}$ be the co-root system with base $\check{\Delta}=\left\{\check{\alpha}_{1}, \ldots, \check{\alpha}_{\ell}\right\}$, where $\check{\alpha}_{i}$ is the co-root corresponding to $\alpha_{i}$. The pairing $\langle$,$\rangle extends$ linearly to the real vector spaces $V=\mathbb{R} \otimes X$ and $\check{V}:=\mathbb{R} \otimes \check{X}$. Thus, a root $\alpha \in R$ can be regarded as the linear functional $\check{v} \mapsto\langle\alpha, \check{v}\rangle$ on $\check{V}$, and by duality $\operatorname{Aut}(R)$ can be regarded as a subgroup of 
$\mathrm{GL}(\check{V})$. In this viewpoint the Weyl group $W$ is the subgroup of $\mathrm{GL}(\check{V})$ generated by the reflections $s_{\alpha}: \check{v} \mapsto \check{v}-\langle\alpha, \check{v}\rangle \check{\alpha}$ for $\alpha \in R$.

Let $\check{\rho}$ be one-half the sum of those co-roots $\check{\alpha} \in \check{R}$ which are non-negative integral combinations of elements of $\check{\Delta}$. We also have

$$
\check{\rho}=\check{\omega}_{1}+\check{\omega}_{2}+\cdots+\check{\omega}_{\ell},
$$

where $\left\{\check{\omega}_{i}\right\}$ are the fundamental co-weights dual to $\Delta$, that is, $\left\langle\alpha_{i}, \check{\omega}_{i}\right\rangle=1$ and $\left\langle\alpha_{i}, \check{\omega}_{j}\right\rangle=0$ if $i \neq j$.

Let $\check{V}^{\vartheta}=\{\check{v} \in \check{V}: \vartheta(\check{v})=\check{v}\}$ be the subspace of $\vartheta$-fixed vectors in $\check{V}$ and let $R_{\vartheta}=\left\{\left.\alpha\right|_{\check{V} \vartheta}: \alpha \in R\right\}$ be the set of restrictions to $\check{V}^{\vartheta}$ of roots in $R$. By duality $\Theta$ permutes the fundamental co-weights $\left\{\check{\omega}_{i}\right\}$, so the vector $\check{\rho}$ lies in $\check{V}^{\vartheta}$. And since $\langle\alpha, \check{\rho}\rangle=1$ for all $\alpha \in \Delta$, it follows that no root vanishes on $\check{V}^{\vartheta}$. Moreover two roots $\alpha, \alpha^{\prime} \in R$ have the same restriction to $\check{V}^{\vartheta}$ if and only if they lie in the same $\langle\vartheta\rangle$-orbit in $R$. Hence we have

$$
R_{\vartheta}=\left\{\beta_{a}: a \in R / \vartheta\right\},
$$

where $R / \vartheta$ is the set of $\langle\vartheta\rangle$-orbits in $R$ and $\beta_{a}=\left.\alpha\right|_{\check{V} \vartheta}$ for any $\alpha \in a$.

For $a \in R / \vartheta$, we define $\check{\beta}_{a} \in \check{V}^{\vartheta}$ by

$$
\check{\beta}_{a}=\left\{\begin{aligned}
\sum_{\alpha \in a} \check{\alpha} & \text { if } \quad 2 \beta_{a} \notin R_{\vartheta} \\
2 \sum_{\alpha \in a} \check{\alpha} & \text { if } \quad 2 \beta_{a} \in R_{\vartheta},
\end{aligned}\right.
$$

and we set $\check{R}_{\vartheta}=\left\{\check{\beta}_{a}: a \in R / \vartheta\right\}$. Then $\left\langle\beta_{a}, \check{\beta}_{a}\right\rangle=2$ and $\left\langle\beta_{a}, \check{\beta}_{b}\right\rangle \in \mathbb{Z}$ for all $a, b \in R / \vartheta$.

Note that $2 \beta_{a} \notin R_{\vartheta}$ precisely when $a$ consists of "orthogonal" roots; that is, when $a=\left\{\gamma_{1}, \ldots, \gamma_{k}\right\}$ with $\left\langle\gamma_{i}, \check{\gamma}_{j}\right\rangle=0$ for $i \neq j$. In this case, the element

$$
s_{a}:=s_{\gamma_{1}} s_{\gamma_{2}} \cdots s_{\gamma_{k}} \in W
$$

has order two, is independent of the order of the product and is centralized by $\vartheta$. If $2 \beta_{a} \in R_{\vartheta}$ we have $a=\left\{\gamma_{1}, \gamma_{2}\right\}$ where $\gamma_{1}+\gamma_{2} \in R$. In this case we define $s_{a}=s_{\gamma_{1}+\gamma_{2}}$, noting this $s_{a}$ is also centralized by $\vartheta$. A short calculation shows that

$$
s_{a}\left(\beta_{b}\right)=\beta_{b}-\left\langle\beta_{b}, \check{\beta}_{a}\right\rangle \beta_{a},
$$

in all cases. On the other hand, if $\beta \in b$, then $s_{a}\left(\beta_{b}\right)=\left.s_{a}(\beta)\right|_{V^{\vartheta}}$, since $s_{a}$ is centralized by $\vartheta$. It follows that $\beta_{b}-\left\langle\beta_{b}, \check{\beta}_{a}\right\rangle \beta_{a} \in R_{\vartheta}$. These involutions $s_{a}$, for $a \in R / \vartheta$, generate the centralizer $W^{\vartheta}=\{w \in W: \vartheta w=w \vartheta\}[30,2.3]$. Thus, $R_{\vartheta}$ is a root system (possibly non-reduced) whose Weyl group is $W^{\vartheta}$. The rank $\ell_{\vartheta}$ of $R_{\vartheta}$ equals the number of $\vartheta$-orbits in $\Delta$.

Let $\mathcal{A}^{\vartheta}$ be an affine space for the vector space $\check{V}^{\vartheta}$. We denote the action by $(v, x) \mapsto v+x$ for $v \in \check{V}^{\vartheta}$ and $x \in \mathcal{A}^{\vartheta}$ and for $x, y \in \mathcal{A}^{\vartheta}$ we let $y-x \in \check{V}^{\vartheta}$ be the unique vector such that $(y-x)+x=y$. For any affine function $\psi: \mathcal{A}^{\vartheta} \rightarrow \mathbb{R}$ we let $\dot{\psi}: \check{V}^{\vartheta} \rightarrow \mathbb{R}$ be the unique linear functional such that $\psi(x+v)=\psi(x)+\langle\dot{\psi}, v\rangle$ for all $v \in \check{V}^{\vartheta}$.

Choose a basepoint $x_{0} \in \mathcal{A}^{\vartheta}$. For each linear functional $\lambda: \check{V}^{\vartheta} \rightarrow \mathbb{R}$ define an affine function $\widetilde{\lambda}: \mathcal{A}^{\vartheta} \rightarrow \mathbb{R}$ by $\widetilde{\lambda}(x)=\left\langle\lambda, x-x_{0}\right\rangle$. In particular, each root $\beta_{a} \in R_{\vartheta}$ gives an affine function $\widetilde{\beta}_{a}$ on $\mathcal{A}^{\vartheta}$.

For each orbit $a \in R / \vartheta$, set $u_{a}=1 /|a|$. If $\beta_{a} \notin 2 R_{\vartheta}$, define

$$
\Psi_{a}=\left\{\widetilde{\beta}_{a}+n u_{a}: n \in \mathbb{Z}\right\}
$$


If $\beta_{a} \in 2 R_{\vartheta}$, define

$$
\Psi_{a}=\left\{\widetilde{\beta}_{a}+\left(n+\frac{1}{2}\right) u_{a}: n \in \mathbb{Z}\right\}
$$

The resulting collection

$$
\Psi(R, \vartheta):=\bigcup_{a \in R / \vartheta} \Psi_{a}
$$

of affine functions on $\mathcal{A}^{\vartheta}$ is a reduced, irreducible affine root system (in the sense of [21, 1.2]) and $x_{0} \in \mathcal{A}^{\vartheta}$ is a special point for $\Psi(R, \vartheta)$.

An alcove in $\mathcal{A}^{\vartheta}$ is a connected component of the open subset of points in $\mathcal{A}$ on which no affine function in $\Psi(R, \vartheta)$ vanishes. There is a unique alcove $C \subset \mathcal{A}^{\vartheta}$ containing $x_{0}$ in its closure and on which $\tilde{\beta}_{a}>0$ for every $\vartheta$-orbit $a \subset \Delta$. The walls of $C$ are hyperplanes $\psi_{i}=0, i=0,1, \ldots, \ell_{\vartheta}=\operatorname{dim} \mathcal{A}^{\vartheta}$, and $\left\{\psi_{0}, \psi_{1}, \ldots, \psi_{\ell_{\vartheta}}\right\}$ is a base of the affine root system $\Psi(R, \vartheta)$. The point $x_{0}$ lies in all but one of these walls; we choose the numbering so that $\psi_{0}\left(x_{0}\right) \neq 0$. There are unique relatively prime positive integers $b_{i}$ such that $\sum b_{i} \dot{\psi}_{i}=0$. We have $b_{0}=1$ and the affine function $\sum_{i=0}^{\ell_{\vartheta}} b_{i} \psi_{i}$ is constant, equal to $1 / e$, where $e=|\vartheta|$. The reflections $r_{i}$ about the hyperplanes $\psi_{i}=0$ for $i=0,1, \ldots, \ell_{\vartheta}$ generate an irreducible affine Coxeter group $W_{\text {aff }}(R, \vartheta)$ which acts simply-transitively on alcoves in $\mathcal{A}^{\vartheta}$.

If $\vartheta=1$ we recover the affine root system attached to $R$ as in [6] and $W_{\text {aff }}(R):=W_{\text {aff }}(R, 1)$ is the affine Weyl group of $R$.

For an example with nontrivial $\vartheta$, take $R$ of type $A_{2}$ and $\vartheta$ of order two. We have $\check{V}=\{(x, y, z) \in$ $\left.\mathbb{R}^{3}: x+y+z=0\right\}$, and

$$
\alpha_{1}=x-y, \quad \alpha_{2}=y-z, \quad \check{\alpha}_{1}=(1,-1,0), \quad \check{\alpha}_{2}=(0,1,-1), \quad \check{\rho}=(1,0,-1) .
$$

The nontrivial automorphism $\vartheta \in \operatorname{Aut}(R)$ permuting $\left\{\alpha_{1}, \alpha_{2}\right\}$ acts on $\check{V}$ by $\vartheta(x, y, z)=(-z,-y,-x)$. We identify $\check{V}^{\vartheta}=\{(x, 0,-x): x \in \mathbb{R}\}$ with $\mathbb{R}$ via projection onto the first component. The $\langle\vartheta\rangle$-orbits in the positive roots are $a=\left\{\alpha_{1}, \alpha_{2}\right\}$ and $b=\left\{\alpha_{1}+\alpha_{2}\right\}$, so $\beta_{a}=x$ and $\beta_{b}=2 x$. If we identify $\mathcal{A}^{\vartheta}=\mathbb{R}$ and take $x_{0}=0$, then

$$
\Psi_{a}=\left\{x+\frac{n}{2}: n \in \mathbb{Z}\right\}, \quad \Psi_{b}=\left\{2 x+n+\frac{1}{2}: n \in \mathbb{Z}\right\} .
$$

The alcove $C$ is the open interval $\left(0, \frac{1}{4}\right)$ in $\mathbb{R}$. The walls of $C$ are defined by the vanishing of the affine roots

$$
\psi_{0}=\frac{1}{2}-2 x, \quad \psi_{1}=x
$$

which satisfy the relation $\psi_{0}+2 \psi_{1}=\frac{1}{2}$, so $b_{0}=1$ and $b_{1}=2$. The group $W_{\text {aff }}(R, \vartheta)$ is infinite dihedral, generated by the reflections of $\mathbb{R}$ about 0 and $\frac{1}{4}$.

We list the affine root systems for nontrivial $\vartheta$ in Table 1 . As the structure of $\Psi(R, \vartheta)$ depends only on $R$ and the order $e$ of $\vartheta$, the pair $(R, \vartheta)$ is indicated by the symbol ${ }^{e} R$, called the type of $(R, \vartheta)$. Information about $\Psi(R, \vartheta)$ is encoded in a twisted affine diagram $D\left({ }^{e} R\right)$ which is a graph with vertices indexed by $i \in\left\{0,1, \ldots, \ell_{\vartheta}\right\}$, labelled by the integers $b_{i}$. The number $m_{i j}$ of bonds between vertices $i$ and $j$ is determined as follows. Choose a $W^{\vartheta}$-invariant inner product $($,$) on V^{\vartheta}$ and suppose that $\left(\dot{\psi}_{j}, \dot{\psi}_{j}\right) \geq\left(\dot{\psi}_{i}, \dot{\psi}_{i}\right)$. Then

$$
m_{i j}=\frac{\left(\dot{\psi}_{j}, \dot{\psi}_{j}\right)}{\left(\dot{\psi}_{i}, \dot{\psi}_{i}\right)}
$$


If $m_{i j}>1$ we put an arrow pointing from vertex $j$ to vertex $i$.

Removing the labels and arrows from the twisted affine diagram $D\left({ }^{e} R\right)$ gives the Coxeter diagram $D\left({ }^{e} R\right)_{\text {cox }}$ of $W_{\text {aff }}(R, \vartheta)$ (except in type ${ }^{2} A_{2}$ the four bonds should be interpreted as $r_{0} r_{1}$ having infinite order). Table 1 gives the twisted affine diagrams for $e>1$ (their analogues for $e=1$ being wellknown). For each type we also give the twisted Coxeter number, which is the sum

$$
h_{\vartheta}=e \cdot\left(b_{0}+b_{1}+\cdots+b_{\ell_{\vartheta}}\right) \text {, }
$$

whose importance will be seen later. The node $i=0$ is indicated by $\bullet$.

Table 1: Twisted Affine diagrams and twisted Coxeter numbers

\begin{tabular}{|c|c|c|c|}
\hline${ }^{e} R$ & $D\left({ }^{e} R\right)$ & $\ell_{\vartheta}$ & $h_{\vartheta}$ \\
\hline${ }^{2} A_{2}$ & $\stackrel{1}{\bullet} \bar{\equiv} \stackrel{2}{\circ}$ & 1 & 6 \\
\hline${ }^{2} A_{2 n}$ & 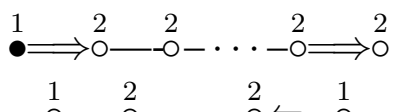 & $n$ & $4 n+2$ \\
\hline${ }^{2} A_{2 n-1}$ & & $n$ & $4 n-2$ \\
\hline${ }^{2} D_{n}$ & 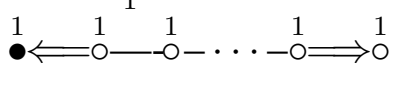 & $n-1$ & $2 n$ \\
\hline${ }^{3} D_{4}$ & $\stackrel{1}{\bullet}-{ }^{2} \Leftarrow{ }^{1}$ & 2 & 12 \\
\hline${ }^{2} E_{6}$ & $\stackrel{1}{2}-{ }^{3} \Longleftarrow{ }^{2} \Longleftarrow{ }^{2}-{ }^{1}$ & 4 & 18 \\
\hline
\end{tabular}

Remark: Let $\mathcal{R}$ be the set of pairs $(R, e)$, where $R$ is an irreducible reduced finite root system and $e$ is a divisor of $|\Theta|$. Let $\mathcal{R}_{\text {aff }}$ be the set of irreducible reduced affine root systems, as in [21], up to isomorphism. Let $\mathcal{D}$ be the set of pairs $(D, o)$, where $D$ is the Coxeter diagram of an irreducible affine Coxeter group and $o$ is a choice of orientation of each multiple edge of $D$. The classification of reduced irreducible affine root systems $[21,1.3]$ shows that the assignments $(R, e) \mapsto{ }^{e} R \mapsto D\left({ }^{e} R\right)$ give bijections

$$
\mathcal{R} \stackrel{\sim}{\longrightarrow} \mathcal{R}_{\mathrm{aff}} \stackrel{\sim}{\longrightarrow} \mathcal{D}
$$

\subsection{Torsion points, Kac coordinates and the normalization algorithm}

Retain the notation of the previous section. Let $\mathcal{A}_{\mathbb{Q}}^{\vartheta}$ be the set of points in $\mathcal{A}^{\vartheta}$ on which the affine roots in $\Psi(R, \vartheta)$ take rational values. The order of a point $x \in \mathcal{A}_{\mathbb{Q}}^{\vartheta}$ is the smallest positive integer $m$ such that $\psi(x) \in \frac{1}{m} \mathbb{Z}$ for every $\psi \in \Psi(R, \vartheta)$. In this case there are integers $s_{i}$ such that $\psi_{i}(x)=s_{i} / m$, and $\operatorname{gcd}\left(s_{0}, \ldots, s_{\ell_{\vartheta}}\right)=1$. Moreover, since $b_{0} \psi_{0}+\cdots+b_{\ell_{\vartheta}} \psi_{\ell_{\vartheta}}$ is constant, equal to $1 / e$, (recall that $e$ is the order of $\vartheta$ ) it follows that

$$
e \cdot \sum_{i=0}^{\ell_{\vartheta}} b_{i} s_{i}=m .
$$

In particular, the order $m$ is divisible by $e$. We call integer vector $\left(s_{0}, s_{1}, \ldots, s_{\ell_{\vartheta}}\right)$ the (un-normalized) Kac coordinates of $x$. 
The point $x$ lies in $\bar{C}$ precisely when all $s_{i}$ are non-negative; in this case we refer to the vector $\left(s_{i}\right)$ as normalized Kac coordinates. The action of the affine Weyl group $W_{\text {aff }}(R, \vartheta)$ on $\mathcal{A}_{\mathbb{Q}}^{\vartheta}$ can be visualized as an action on Kac coordinates, as follows. The reflection $r_{j}$ about the wall $\psi_{j}=0$ sends the Kac coordinates $\left(s_{i}\right)$ to $\left(s_{i}^{\prime}\right)$, where

$$
s_{i}^{\prime}=s_{i}-\left\langle\beta_{i}, \check{\beta}_{j}\right\rangle s_{j}
$$

Un-normalized Kac coordinates may have some $s_{j}<0$. If we apply $r_{j}$ and repeat this process by selecting negative nodes and applying the corresponding reflections, we will eventually obtain normalized Kac coordinates $\left(s_{i}^{\prime}\right)$. Geometrically, this normalization algorithm amounts to moving a given point $x \in \mathcal{A}_{\mathbb{Q}}^{\Theta}$ into the fundamental alcove $\bar{C}$ by a sequence of reflections about walls, see [24, Sec. 3.2]. We have implemented the normalization algorithm on a computer and used it extensively to construct the tables in sections 9 and 11 .

The image of the projection $e^{-1} \sum_{i=0}^{e-1} \vartheta^{i}: \check{X} \rightarrow V^{\vartheta}$ is a lattice $Y_{\vartheta}$ in $V^{\vartheta}$ which is preserved by $W^{\vartheta}$. The extended affine Weyl group

$$
\widetilde{W}_{\text {aff }}(R, \vartheta):=W^{\vartheta} \rtimes Y_{\vartheta}
$$

contains $W_{\text {aff }}(R, \vartheta)$ as a normal subgroup of finite index and the quotient may be identified with a group of symmetries of the oriented diagram $D\left({ }^{e} R\right)$. We regard two normalized Kac diagrams as equivalent if one is obtained from the other by a symmetry of the oriented diagram $D\left({ }^{e} R\right)$ coming from $\widetilde{W}_{\text {aff }}(R, \vartheta)$. For $R=E_{6}, E_{7}, E_{8}$ and $e=1$ these diagram symmetries are: rotation of order three, reflection of order two and trivial, respectively. In type ${ }^{2} E_{6}$ these diagram symmetries are trivial (see table (3)).

\section{$2.3 \mu_{m}$-actions on Lie algebras}

Let $k$ be an algebraically closed field. All $k$-algebras are understood to be commutative with 1 , and in this section all group schemes are affine over $k$, and are regarded as representable functors from the category of finitely generated $k$-algebras to the category of groups. We refer to [36] for more details on affine group schemes.

Every finitely generated $k$-algebra $A$ is a direct product of $k$-algebras $A=\prod_{\iota \in I(A)} A_{\iota}$, where $I(A)$ indexes the connected components $\operatorname{Spec}\left(A_{\iota}\right)$ of $\operatorname{Spec}(A)$ and each $A_{\iota}$ is a $k$-algebra with no non-trivial idempotents. This decomposition is to be understood when we describe the $A$-valued points in various group schemes below. Each finite (abstract) group $\Gamma$ is regarded a constant group scheme, given by $\Gamma(A)=\prod_{\iota \in I(A)} \Gamma\left(A_{\iota}\right)$, where $\Gamma\left(A_{\iota}\right)=\Gamma$. In other words, an element $\gamma \in \Gamma(A)$ is a function $\left(\iota \mapsto \gamma_{\iota}\right)$ from $I(A)$ to $\Gamma$.

Let $\boldsymbol{\mu}_{m}$ denote the group scheme of $m^{\text {th }}$ roots of unity, whose $A$-valued points are given by

$$
\boldsymbol{\mu}_{m}(A)=\left\{a \in A: a^{m}=1\right\}=\prod_{\iota \in I(A)} \boldsymbol{\mu}_{m}\left(A_{\iota}\right)
$$

If $m$ is nonzero in $k$ then $\boldsymbol{\mu}_{m}\left(A_{\iota}\right)=\boldsymbol{\mu}_{m}(k)$ for every $\iota \in I(A)$, so $\boldsymbol{\mu}_{m}$ is a constant group scheme and we have

$$
\boldsymbol{\mu}_{m}(A)=\prod_{\iota \in I(A)} \boldsymbol{\mu}_{m}(k)
$$


If $m$ is zero in $k$ then $\boldsymbol{\mu}_{m}$ is not a constant group scheme.

A $k$-vector space $V$ can be regarded as a $k$-scheme such that $V(A)=A \otimes_{k} V$. To give a grading $V=\sum_{i \in \mathbb{Z} / m \mathbb{Z}} V_{i}$ as $k$-schemes is to give a morphism $\varrho: \boldsymbol{\mu}_{m} \rightarrow \mathrm{GL}(V)$, where $\mathrm{GL}(V)(A)$ is the automorphism group of the free $A$-module $V(A)$. Indeed, $\mathbb{Z} / m$ is canonically isomorphic to the Cartier dual $\operatorname{Hom}\left(\boldsymbol{\mu}_{m}, \mathbf{G}_{m}\right)$, so a morphism $\varrho: \boldsymbol{\mu}_{m} \rightarrow \mathrm{GL}(V)$ gives a grading $V(A)=\bigoplus_{i \in \mathbb{Z} / m} V_{i}(A)$ where $V_{i}(A)=\left\{v \in V(A): \varrho(\zeta) v=\zeta^{i} v \quad \forall \zeta \in \boldsymbol{\mu}_{m}(A)\right\}$.

Now let $R$ be an irreducible root system as before, with base $\Delta$ and group of based automorphisms $\Theta$. Set $X=\mathbb{Z} R$ and $\check{X}=\operatorname{Hom}(X, \mathbb{Z})$. Then $(X, R, \check{X}, \check{R})$ is the root datum of a connected simple algebraic group scheme $G$ over $k$ of adjoint type. Let $\mathfrak{g}$ be the Lie algebra of $G$ and let $T \subset B$ be a maximal torus contained in a Borel subgroup of $G$. We identify $R$ with the set of roots of $T$ in $\mathfrak{g}$, and $\Delta$ with the set of simple roots of $T$ in the Lie algebra of $B$. Choose a root vector $E_{i}$ for each simple root $\alpha_{i} \in \Delta$. The data $\left(X, R, \check{X}, \check{R},\left\{E_{i}\right\}\right)$ is called a pinning of $G$.

Fix an element $\vartheta \in \Theta$. Assume the order $e$ of $\vartheta$ is nonzero in $k$, so that $\boldsymbol{\mu}_{e}$ and $\langle\vartheta\rangle$ are isomorphic constant group schemes over $k$, and choose an isomorphism $\tau: \boldsymbol{\mu}_{e} \rightarrow\langle\vartheta\rangle$.

By our choice of pinning $\left(X, R, \check{X}, \check{R},\left\{E_{i}\right\}\right)$, the group $\langle\vartheta\rangle$ may also be regarded as a subgroup of $\operatorname{Aut}(\mathfrak{g})$ permuting the root vectors $E_{i}$ in the same way $\vartheta$ permutes the roots $\alpha_{i}$, and we have a semidirect product

$$
G \rtimes\langle\vartheta\rangle \subset \operatorname{Aut}(\mathfrak{g}),
$$

where the cyclic group $\langle\vartheta\rangle$ is now viewed as a constant subgroup scheme of automorphisms of $\mathfrak{g}$, whose points in each $k$-algebra $A$ consist of vectors $\left(\vartheta^{n_{\iota}}\right)$ acting on $\mathfrak{g}(A)=\prod_{\iota} \mathfrak{g}\left(A_{\iota}\right)$, with $\vartheta^{n_{\iota}}$ acting on the factor $\mathfrak{g}\left(A_{\iota}\right)$.

Now let $m$ be a positive integer divisible by $e$ (but $m$ could be zero in $k$ ). Let $m / e: \boldsymbol{\mu}_{m} \rightarrow \boldsymbol{\mu}_{e}$ be the morphism sending $\zeta \in \boldsymbol{\mu}_{m}(A)$ to $\zeta^{m / e} \in \boldsymbol{\mu}_{e}(A)$ for every $k$-algebra $A$.

Finally, for each rational point $x \in \mathcal{A}_{\mathbb{Q}}^{\vartheta}$ of order $m$ we shall now define a morphism

$$
\varrho_{x}: \boldsymbol{\mu}_{m} \rightarrow T^{\vartheta} \times\langle\vartheta\rangle
$$

where $T^{\vartheta}$ is the subscheme of $\vartheta$-fixed points in $T$. We have $x=\frac{1}{m} \check{\lambda}+x_{0}$, for some $\check{\lambda} \in \check{X}^{\vartheta}$. The co-character $\check{\lambda}$ restricts to a morphism $\check{\lambda}_{m}: \boldsymbol{\mu}_{m} \rightarrow T^{\vartheta}$ and we define $\varrho_{x}$ on $A$-valued points by

$$
\varrho_{x}(\zeta)=\check{\lambda}_{m}(\zeta) \times \tau\left(\zeta^{m / e}\right), \quad \text { for } \quad \zeta \in \boldsymbol{\mu}_{m}(A) .
$$

Since

$$
\operatorname{Hom}\left(\boldsymbol{\mu}_{m}, T^{\vartheta}\right)=\check{X}^{\vartheta} / m \check{X}^{\vartheta} \simeq \frac{1}{m} \check{X}^{\vartheta} / \check{X}^{\vartheta},
$$

we see that $\check{\lambda}_{m}$ corresponds precisely to an orbit of $x$ under translation by $\check{X}^{\vartheta}$ on $\mathcal{A}_{\mathbb{Q}}^{\vartheta}$. The condition that $x$ has order $m$ means that $\check{\lambda}_{m}$ does not factor through $\boldsymbol{\mu}_{d}$ for any proper divisor $d \mid m$.

Let $\widetilde{w} \in \widetilde{W}_{\text {aff }}(R, \vartheta)$ have projection $w \in W^{\vartheta}$ and denote the canonical action of $W^{\vartheta}$ on $T^{\vartheta}$ by $w \cdot t$, for $t \in T^{\vartheta}(A)$. Then we have

$$
\varrho_{\widetilde{w} \cdot x}(\zeta)=w \cdot \varrho_{x}(\zeta)
$$

for all $\zeta \in \boldsymbol{\mu}_{m}(A)$. One can check (cf. [24, section 3]) that two points $x, y \in \mathcal{A}_{\mathbb{Q}}^{\vartheta}$ of order $m$ give $G$-conjugate embeddings $\varrho_{x}, \varrho_{y}: \boldsymbol{\mu}_{m} \hookrightarrow T^{\vartheta} \times \vartheta$ if and only if $x$ and $y$ are conjugate under $\widetilde{W}_{\text {aff }}(R, \vartheta)$. 
The morphism $\varrho_{x}$ is thus determined by the Kac coordinates $\left(s_{0}, s_{1}, \ldots, s_{\ell_{\vartheta}}\right)$ of $x$ and the $G$-conjugacy class of $\varrho_{x}$ is determined by the normalized Kac coordinates of the $\widetilde{W}_{\text {aff }}(R, \vartheta)$-orbit of $x$.

\subsection{Principal $\mu_{m}$-actions}

We continue with the notation of section 2.3. Recall that $\check{\rho} \in \check{X}^{\vartheta}$ is the sum of the fundamental co-weights $\check{\omega}_{i}$. For every positive integer $m$ divisible by $e$, we have a principal point

$$
x_{m}:=x_{0}+\frac{1}{m} \check{\rho} \in \mathcal{A}_{\mathbb{Q}}^{\vartheta}
$$

of order $m$. It corresponds to the principal embedding

$$
\varrho_{m}=\varrho_{x_{m}}: \boldsymbol{\mu}_{m} \longrightarrow T^{\vartheta} \times\langle\vartheta\rangle, \quad \text { given by } \quad \varrho_{m}(\zeta)=\check{\rho}(\zeta) \times \tau\left(\zeta^{m / e}\right) .
$$

The Kac coordinates of $x_{m}$ and $\varrho_{m}$ are given as follows. If $1 \leq i \leq \ell_{\vartheta}$ we have $\psi_{i}=\tilde{\beta}_{i}$ for some $\beta_{i} \in R_{\vartheta}$ which is the restriction to $\check{V}^{\Theta}$ of a simple root $\alpha_{i} \in \bar{\Delta}$. Since $\left\langle\alpha_{i}, \check{\rho}\right\rangle=1$, it follows that $\left\langle\psi, x_{m}\right\rangle=1 / m$ so $s_{i}=1$, and we have

$$
m=e \cdot \sum_{i=0}^{\ell_{\vartheta}} b_{i} s_{i}=e s_{0}+e \cdot \sum_{i=1}^{\ell_{\vartheta}} b_{i}=e s_{0}+h_{\vartheta}-e
$$

where $h_{\vartheta}=e \cdot \sum_{i=0}^{\ell_{\vartheta}} b_{i}$ is the twisted Coxeter number of $R_{\vartheta}$ (see (2)). Hence the remaining Kaccoordinate of the principal point $x_{m}$ is

$$
s_{0}=1+\frac{m-h_{\vartheta}}{e}
$$

This is negative if $m<h_{\vartheta}-e$, in which case we can apply the normalization algorithm of section 2.2 to obtain the normalized Kac coordinates of $x_{m}$. Examples are found in the tables of section 8.1.

We will be especially interested in the points $x_{m}$ where $m$ is the order of an elliptic $\mathbb{Z}$-regular automorphism in $W \vartheta$ (defined in the next section). The twisted Coxeter number $h_{\vartheta}$ is one of these special values of $m$, corresponding to $s_{0}=1$ (cf. section 8 below).

\section{$3 \mathbb{Z}$-regular automorphisms of root systems}

We continue with the notation of section 2.1: $R$ is an irreducible finite reduced root system with a chosen base $\Delta$ and automorphism group $\operatorname{Aut}(R)=W \rtimes \Theta$, where $W$ is the Weyl group of $R$ and $\Theta$ is the subgroup of $\operatorname{Aut}(R)$ preserving $\Delta$.

Definition 3.1 An automorphism $\sigma \in \operatorname{Aut}(R)$ is $\mathbb{Z}$-regular if the group generated by $\sigma$ acts freely on $R$. 
This is nearly equivalent to Springer's notion of a regularity (over $\mathbb{C}$ ) [29]. In this section we will reconcile our definition with that of Springer.

Let $X=\mathbb{Z} R$ be the root lattice of $R$ and let $\check{X}=\operatorname{Hom}(X, \mathbb{Z})$ be the co-weight lattice. We say that a vector $\check{v} \in k \otimes \check{X}$ is $\boldsymbol{k}$-regular if $\langle\alpha, \breve{v}\rangle \neq 0$ for every $\alpha \in R$. We say also that an automorphism $\sigma \in \operatorname{Aut}(R)$ is $\boldsymbol{k}$-regular if $\sigma$ has a $k$-regular eigenvector in $k \otimes \check{X}$. Taking $k=\mathbb{C}$ we recover Springer's definition of regularity [29].

At first glance it appears that $\sigma$ could be $k$-regular for some fields $k$ but not others. This is why we have defined regularity over $\mathbb{Z}$, as in Def. 3.1. Of course the definition of $\mathbb{Z}$-regularity seems quite different from that of $k$-regularity. An argument due to Kostant for the Coxeter element (cf. [14, Cor. 8.2]) shows that a $k$-regular automorphism is $\mathbb{Z}$-regular (see [29, Prop. 4.10]). The converse is almost true but requires an additional condition. We will prove:

Proposition 3.2 An automorphism $\sigma \in \operatorname{Aut}(R)$ is $\mathbb{Z}$-regular if and only if for every algebraically closed field $k$ in which the order $m$ of $\sigma$ is nonzero there is $k$-regular eigenvector for $\sigma$ in $k \otimes \check{X}$ whose eigenvalue has order $m$.

Suppose $\sigma=w \vartheta$ where $w \in W$ and $\vartheta \in \Theta$ is a based automorphism of order $e$. If $\sigma$ has order $m$ and has a $k$-regular eigenvalue $\lambda$ of order $d$, then $m=\operatorname{lcm}(d, e)$. Indeed, it is clear that $m$ is divisible by $n:=\operatorname{lcm}(d, e)$. Conversely, we have $\lambda^{n}=1$ so $\sigma^{n}$ fixes a regular vector, but $\sigma^{n} \in W$, so in fact $\sigma^{n}=1$ and $m \mid n$. Hence the notions of $\mathbb{Z}$-regularity and $k$-regularity coincide precisely when $e \mid d$. In particular they coincide if $\vartheta=1$, that is, if $\sigma \in W$. However, if $\vartheta$ has order $e>1$ and we take $\sigma=\vartheta$, then $\sigma$ fixes the $k$-regular vector $\check{\rho}$ so $\sigma$ is $k$-regular (if $e \neq 0$ in $k$ ). However $\sigma$ fixes the highest root, so $\sigma$ is not $\mathbb{Z}$-regular. And if $\zeta \in k^{\times}$has order $e$ there are no $k$-regular vectors in the $\zeta$-eigenspace of $\sigma$.

The proof of Prop. 3.2 will be given after some preliminary lemmas.

Lemma 3.3 An automorphism $\sigma \in \operatorname{Aut}(R)$ is based if and only if no root of $R$ vanishes on $\check{X}^{\sigma}$.

Proof: Assume that $\sigma \in \operatorname{Aut}(R)$ preserves a base $\Delta^{\prime} \subset R$. Then $\sigma$ preserves the set $R^{+}$of roots in $R$ which are non-negative integral linear combinations of roots in $\Delta^{\prime}$. The vector $\sum_{\beta \in R^{+}} \check{\beta}$ belongs to $\check{X}^{\sigma}$ and no root vanishes on it.

Conversely, let $\check{v} \in \check{X}^{\sigma}$ be a vector on which no root in $R$ vanishes. Then $v$ defines a chamber $\mathcal{C}$ in the real vector space $\mathbb{R} \otimes X$, namely,

$$
\mathcal{C}=\{\lambda \in \mathbb{R} \otimes X:\langle\lambda, \check{v}\rangle>0\} .
$$

As $\sigma$ fixes $\check{v}$, the chamber $\mathcal{C}$ is preserved $\sigma$, so $\sigma$ permutes the walls of $\mathcal{C}$. The set of roots $\alpha$ for which ker $\check{\alpha}$ is a wall of $\mathcal{C}$ is therefore a base of $R$ preserved by $\sigma$.

Next, we say that $\sigma \in \operatorname{Aut}(R)$ is primitive if $\sigma$ preserves no proper root subsystem of $R$.

Lemma 3.4 If $\sigma \in \operatorname{Aut}(R)$ is primitive, then its characteristic polynomial on $V$ is irreducible over $\mathbb{Q}$. That is, we have $\operatorname{det}\left(t I_{V}-\left.\sigma\right|_{V}\right)=\Phi_{m}(t)$, where $m$ is the order of $\sigma$ and $\Phi_{m}(t) \in \mathbb{Z}[t]$ is the cyclotomic polynomial whose roots are the primitive $m^{\text {th }}$ roots of unity. 
Proof: In this proof we change notation slightly and let $V=\mathbb{Q} \otimes X$ denote the rational span of $X$ and let $\overline{\mathbb{Q}}$ be an algebraic closure of $\mathbb{Q}$.

For $\alpha \in R$, let $V_{\alpha} \subset V$ be the rational span of the $\sigma$-orbit of $\alpha$. Since $V_{\alpha}$ is spanned by roots, it follows from [6, VI.1] that $R \cap V_{\alpha}$ is a root subsystem of $R$. As it is preserved by the primitive automorphism $\sigma$, we must have $R \subset V_{\alpha}$, so $V_{\alpha}=V$. Hence the map $\mathbb{Q}[t] \rightarrow V$ given by sending $f(t) \mapsto f(\sigma) \alpha$ is surjective, and its kernel is the ideal in $\mathbb{Q}[t]$ generated by the minimal polynomial $M(t)$ of $\sigma$ on $V$. Hence $\operatorname{deg} M(t)=\operatorname{dim} V$ so we have $M(t)=\operatorname{det}\left(t I_{V}-\left.\sigma\right|_{V}\right)$.

We must show that $M(t)$ is irreducible over $\mathbb{Q}$. If not, then $M(t)$ is divisible by $\Phi_{d}(t)$ for some proper divisor $d \mid m$. This means $\sigma$ has an eigenvalue of order $d$ on $\overline{\mathbb{Q}} \otimes V$, implying that $\sigma^{d}$ has nonzero fixed-point space $\check{X}^{\sigma^{d}}$. The set of roots vanishing on $\check{X}^{\sigma^{d}}$ is a root subsystem not equal to the whole of $R$, and therefore is empty, again using the primitivity of $\sigma$.

By Lemma 3.3, $\sigma^{d}$ is a nontrivial automorphism preserving a base $\Delta^{\prime}$ of $R$. As in the proof of that lemma, the sum of the positive roots for $\Delta^{\prime}$ is a nonzero $\overline{\mathbb{Q}}$-regular vector in $V$ fixed by $\sigma^{d}$. Hence the nontrivial subgroup $\left\langle\sigma^{d}\right\rangle$ has trivial intersection with $W$. If $\sigma \in W$ this is a contradiction and the lemma is proved in this case.

Assume that $\sigma \notin W$. Since $R$ is irreducible and we have shown that the projection $\operatorname{Aut}(R) \rightarrow \Theta$ is injective on $\left\langle\sigma^{d}\right\rangle$, it follows that $\sigma^{d}$ has order $e \in\{2,3\}$. We must also have $(e, d)=1$ and $m=e d$. As $e$ is determined by the projection of $\sigma$ to $\Theta$, it follows that $d$ is the unique proper divisor of $m$ such that $\Phi_{d}(t)$ divides $M(t)$. Since the roots of $M(t)$ are $m^{t h}$ roots of unity (because $\sigma^{m}=1$ ) and are distinct (since $\sigma$ is diagonalizable on $\overline{\mathbb{Q}} \otimes V$ ) and $M(t) \neq \Phi_{d}(t)$ by assumption, it follows that $M(t)=\Phi_{m}(t) \cdot \Phi_{d}(t)$.

If $e=2$ then $-\sigma \in W$ is also primitive, with reducible minimal polynomial $M(-t)=\Phi_{m}(-t)$. $\Phi_{d}(-t)$, contradicting the case of the lemma previously proved. If $e=3$, then $\Phi$ has type $D_{4}$, so $m=3 d$ and

$$
4=\operatorname{deg} M=\phi(3 d)+\phi(d)=\phi(d)[\phi(3)+1]=3 \phi(d),
$$

which is also impossible. The lemma is now proved in all cases.

Now let $\sigma \in \operatorname{Aut}(R)$ be a $\mathbb{Z}$-regular automorphism of order $m$. Recall from Def. 3.1 that this means the group $\langle\sigma\rangle$ generated by $\sigma$ acts freely on $R$. For each $\alpha \in R$, let $V_{\alpha} \subset \mathbb{Q} \otimes X$ denote the $\mathbb{Q}$-span of the $\langle\sigma\rangle$-orbit of $\alpha$ and let $M_{\alpha}(t)$ be the minimal polynomial of $\sigma$ on $V_{\alpha}$.

Lemma 3.5 If $\sigma$ is $\mathbb{Z}$-regular of order $m$ then $\Phi_{m}(t)$ divides $M_{\alpha}(t)$ in $\mathbb{Z}[t]$, for all $\alpha \in R$.

Proof: Let $\zeta \in \overline{\mathbb{Q}}^{\times}$be a root of unity of order $m$ and let $\alpha \in R$. It suffices to show that $\zeta$ is an eigenvalue of $\sigma$ in $\overline{\mathbb{Q}} \otimes V_{\alpha}$. Let $R^{\prime}$ be a minimal (nonempty) $\sigma$-stable root subsystem of $R \cap V_{\alpha}$, and let

$$
R^{\prime}=R_{0}^{\prime} \cup R_{1}^{\prime} \cup \cdots \cup R_{k-1}^{\prime}
$$

be the decomposition of $R^{\prime}$ into irreducible components. These are permuted transitively by $\sigma$; we index them so that $\sigma^{i} R_{0}^{\prime}=R_{i}^{\prime}$ for $i \in \mathbb{Z} / k$. The stabilizer of $R_{0}^{\prime}$ in $\langle\sigma\rangle$ is generated by $\sigma^{k}$. Correspondingly, the rational span $V^{\prime}$ of $R^{\prime}$ is a direct sum

$$
V^{\prime}=V_{0}^{\prime} \oplus V_{1}^{\prime} \oplus \cdots \oplus V_{k-1}^{\prime} \subset V_{\alpha}
$$


where $V_{i}^{\prime}$ is the rational span of $R_{i}^{\prime}$.

Suppose that $\eta:=\zeta^{k}$ is an eigenvalue of $\tau:=\sigma^{k}$ in $\overline{\mathbb{Q}} \otimes V_{0}^{\prime}$, afforded by the vector $v \in \overline{\mathbb{Q}} \otimes V_{0}^{\prime}$. Let $S$ and $T$ denote the group algebras over $\overline{\mathbb{Q}}$ of $\langle\sigma\rangle$ and $\langle\tau\rangle$, respectively, and let $\overline{\mathbb{Q}}_{\eta}$ be the $T$-module with underlying vector space $\overline{\mathbb{Q}}$ on which $\tau$ acts as multiplication by $\eta$. There is a unique map of $S$-modules

$$
f: S \otimes_{T} \overline{\mathbb{Q}}_{\eta} \longrightarrow V^{\prime}
$$

such that $f(1 \otimes 1)=v \in V_{0}^{\prime}$. As $f\left(\sigma^{i} \otimes 1\right)=\sigma^{i} v \in \overline{\mathbb{Q}} \otimes V_{i}^{\prime}$, and the spaces $V_{0}^{\prime}, V_{1}^{\prime}, \ldots, V_{k-1}^{\prime}$ are linearly independent, it follows that $f$ is injective. Frobenius reciprocity implies that $\zeta$ appears as an eigenvalue of $\sigma$ in $\overline{\mathbb{Q}} \otimes V^{\prime}$, hence also in $\overline{\mathbb{Q}} \otimes V_{\alpha}$.

It therefore suffices to prove that $\eta$ appears as an eigenvalue of $\tau$ on $\overline{\mathbb{Q}} \otimes V_{0}^{\prime}$. Since $\sigma$ acts freely on $R$, it follows that $\tau$ acts freely on $R_{0}^{\prime}$ and has order $n:=m / k$ on $R_{0}^{\prime}$. We claim that $\tau$ is primitive on $R_{0}^{\prime}$. For if $R^{\prime \prime} \subset R_{0}^{\prime}$ is a root subsystem preserved by $\tau$ then $R^{\prime \prime} \cup \sigma R^{\prime \prime} \cup \cdots \cup \sigma^{k-1} R^{\prime \prime}$ is a root subsystem preserved by $\sigma$ which must equal $R^{\prime}$ (by minimality), so that $R^{\prime \prime}=R_{0}^{\prime}$. Hence $\tau$ is indeed primitive on $R_{0}^{\prime}$. By Lemma 3.4 the characteristic polynomial of $\tau$ on $V_{0}^{\prime}$ is the cyclotomic polynomial $\Phi_{n}(t)$, which has the root $\zeta^{m / n}=\zeta^{k}=\eta$. Therefore $\eta$ appears as an eigenvalue of $\tau$ on $\overline{\mathbb{Q}} \otimes V_{0}^{\prime}$, as desired.

We are now ready to prove Prop. 3.2. Let $k$ be an algebraically closed field and set $V_{k}:=k \otimes X$, $\check{V}_{k}:=k \otimes \check{X}$. Recall that a $k$-regular vector $\check{v} \in \check{V}_{k}$ is one for which $\langle\alpha, \check{v}\rangle \neq 0$ for all $\alpha \in R$.

For completeness we recall the proof of the easy direction of Prop. 3.2 (cf. [29, 4.10]). Assume that $\sigma \in \operatorname{Aut}(R)$ is $k$-regular, and let $\check{v} \in \check{V}_{k}$ be a $k$-regular eigenvector of $\sigma$ with eigenvalue $\zeta \in k^{\times}$of order $m$ equal to the order of $\sigma$. Suppose $\sigma^{d} \alpha=\alpha$ for some $\alpha \in R$. Then

$$
0 \neq\langle\alpha, \check{v}\rangle=\left\langle\sigma^{d} \alpha, \check{v}\right\rangle=\left\langle\alpha, \sigma^{-d} \check{v}\right\rangle=\zeta^{-d}\langle\alpha, \check{v}\rangle .
$$

It follows that $\zeta^{d}=1$. Since $\sigma$ and $\zeta$ have the same order, it follows that $\sigma^{d}=1$. Hence $\langle\sigma\rangle$ acts freely on $R$, so $\sigma$ is $\mathbb{Z}$-regular.

Assume now that $\sigma$ is $\mathbb{Z}$-regular, so that $\langle\sigma\rangle$ acts freely on $R$. Let $\bar{\Phi}_{m}(t)$ denote the image, under the map $\mathbb{Z}[t] \rightarrow k[t]$ induced by the canonical map $\mathbb{Z} \rightarrow k$, of the cyclotomic polynomial $\Phi_{m}(t)$. Since $m$ is nonzero in $k$, it follows that all roots of $\bar{\Phi}_{m}(t)$ in $k$ have order $m$. Let $\zeta \in k^{\times}$be one of them.

Let $\alpha \in R$ and let $X_{\alpha}$ be the subgroup of $X$ generated by the $\langle\sigma\rangle$-orbit of $\alpha$. Then $X_{\alpha}$ is a lattice in $V_{\alpha}=\mathbb{Q} \otimes X_{\alpha}$ and $\Phi_{m}(t)$ divides the characteristic polynomial $\operatorname{det}\left(t I-\left.\sigma\right|_{X_{\alpha}}\right)$ in $\mathbb{Z}[t]$, by Lemma 3.5. Hence $\bar{\Phi}_{m}(t)$ divides $\operatorname{det}\left(t I-\left.\sigma\right|_{k \otimes X_{\alpha}}\right)$ in $k[t]$. In particular $\zeta^{-1}$ is an eigenvalue of $\sigma$ on $k \otimes X_{\alpha}$.

The operator $P_{\zeta} \in \operatorname{End}\left(V_{k}\right)$ given by

$$
P_{\zeta}=1+\zeta \sigma+\zeta^{2} \sigma^{2}+\cdots+\zeta^{m-1} \sigma^{m-1}
$$

preserves $k \otimes X_{\alpha}$ and $P_{\zeta}\left(k \otimes X_{\alpha}\right)$ is the $\zeta^{-1}$-eigenspace of $\sigma$ in $k \otimes X_{\alpha}$. As $X_{\alpha}$ is spanned by roots $\sigma^{i} \alpha$ and $P_{\zeta}\left(\sigma^{i} \alpha\right)=\sigma^{-i} P_{\zeta}(\alpha)$, it follows that $P_{\zeta}(\alpha) \neq 0$.

As $\alpha \in R$ was arbitrary, we have that $P_{\zeta}(\alpha) \neq 0$ for all $\alpha \in R$. Since $k$ is infinite, there exists $\check{v} \in \check{V}_{k}$ such that $\left\langle P_{\zeta}(\alpha), \check{v}\right\rangle \neq 0$ for all $\alpha \in R$.

The dual projection

$$
\check{P}_{\zeta}=1+\zeta^{-1} \sigma+\zeta^{-2} \sigma^{2}+\cdots+\zeta^{1-m} \sigma^{m-1} \in \operatorname{End}\left(\check{V}_{k}\right)
$$


satisfies

$$
\left\langle\alpha, \check{P}_{\zeta}(\check{v})\right\rangle=\left\langle P_{\zeta}(\alpha), \check{v}\right\rangle \neq 0
$$

for all $\alpha \in R$. Therefore $\check{P}_{\zeta}(\check{v})$ is a $k$-regular eigenvector of $\sigma$ in $\check{V}_{k}$ whose eigenvalue $\zeta$ has order $m$. This completes the proof of Prop. 3.2.

\section{Positive rank gradings}

Let $\mathfrak{g}$ be the Lie algebra of a connected simple algebraic group $G$ of adjoint type over an algebraically closed field $k$ whose characteristic is not a torsion prime for $G$. Then $G=\operatorname{Aut}(\mathfrak{g})^{\circ}$ is the identity component of Aut $(\mathfrak{g})$. We fix a Cartan subalgebra $\mathfrak{t}$ of $\mathfrak{g}$ with corresponding maximal torus $T=C_{G}(\mathfrak{t})$ and let $R$ be the set of roots of $\mathfrak{t}$ of $\mathfrak{g}$. Let $N=N_{G}(T)$ be the normalizer of $T$, so that $W=N / T$ is the Weyl group of $R$.

From now on we only consider gradings $\mathfrak{g}=\oplus_{i \in \mathbb{Z} / m} \mathfrak{g}_{i}$ whose period $m$ is nonzero in $k$. By choosing an $m^{t h}$ root of unity in $k^{\times}$, we get an automorphism $\theta \in \operatorname{Aut}(\mathfrak{g})$ of order $m$, such that $\theta$ acts on $\mathfrak{g}_{i}$ by the scalar $\zeta^{i}$.

In this section we show how all such gradings of positive rank may be effectively found by computing lifts to $\operatorname{Aut}(\mathfrak{g})$ of automorphisms $\sigma \in \operatorname{Aut}(R)$.

\subsection{A canonical Cartan subalgebra}

Given any Cartan subalgebra $\mathfrak{s}$ of $\mathfrak{g}$ with centralizer $S=C_{G}(\mathfrak{s})$, let

$$
\operatorname{Aut}(\mathfrak{g}, \mathfrak{s})=\{\theta \in \operatorname{Aut}(\mathfrak{g}): \theta(\mathfrak{s})=\mathfrak{s}\} .
$$

We have an isomorphism (obtained by conjugating $\mathfrak{s}$ to our fixed Cartan subalgebra $\mathfrak{t}$ )

$$
\operatorname{Aut}(\mathfrak{g}, \mathfrak{s}) / S \simeq \operatorname{Aut}(R)
$$

which is unique up to conjugacy in $\operatorname{Aut}(R)$. Thus any element of $\operatorname{Aut}(\mathfrak{g}, \mathfrak{s})$ gives a well-defined conjugacy class in $\operatorname{Aut}(R)$. However, an automorphism $\theta \in \operatorname{Aut}(\mathfrak{g})$ may normalize various Cartan subalgebras $\mathfrak{s}$, giving rise to various classes in $\operatorname{Aut}(R)$. We will define a canonical $\theta$-stable Cartan subalgebra, which will allow us associate to $\theta$ a well-defined conjugacy class in $\operatorname{Aut}(R)$.

For each $\theta \in \operatorname{Aut}(\mathfrak{g})$ whose order is nonzero in $k$ we define a canonical $\theta$-stable Cartan subalgebra $\mathfrak{s}$ of $\mathfrak{g}$ as follows. Let $\mathfrak{c} \subset \mathfrak{g}_{1}$ be a Cartan subspace. The centralizer $\mathfrak{m}=\mathfrak{z}_{\mathfrak{g}}(\mathfrak{c})$ is a $\theta$-stable Levi subalgebra of $\mathfrak{g}$ and we have $\mathfrak{m}=\oplus \mathfrak{m}_{i}$ where $\mathfrak{m}_{i}=\mathfrak{m} \cap \mathfrak{g}_{i}$. Choose a Cartan subalgebra $\mathfrak{s}_{0}$ of $\mathfrak{m}_{0}$. Then $\mathfrak{s}_{0}$ contains regular elements of $\mathfrak{m}[19$, Lemma 1.3], so the centralizer

$$
\mathfrak{s}:=\mathfrak{z}_{\mathfrak{m}}\left(\mathfrak{s}_{0}\right)
$$

is a $\theta$-stable Cartan subalgebra of $\mathfrak{m}$, and $\mathfrak{s}$ is also a Cartan subalgebra of $\mathfrak{g}$. We have $\mathfrak{s} \cap \mathfrak{g}_{0}=\mathfrak{s}_{0}$ (so our notation is consistent) and $\mathfrak{s} \cap \mathfrak{g}_{1}=\mathfrak{c}$. Since $G_{0}$ is transitive on Cartan subspaces in $\mathfrak{g}_{1}[19$, Thm. 2.5] and $C_{G_{0}}(\mathfrak{c})^{\circ}$ is transitive on Cartan subalgebras of its Lie algebra $\mathfrak{m}_{0}$, the Cartan subalgebra $\mathfrak{s}$ is unique up to $G_{0}$-conjugacy. 


\subsection{A relation between $\operatorname{Aut}(\mathfrak{g})$ and $\operatorname{Aut}(R)$}

For $\theta \in \operatorname{Aut}(\mathfrak{g})$ and $\sigma \in \operatorname{Aut}(R)$ we write

$$
\theta \vdash \sigma
$$

if the following two conditions are fulfilled:

- $\theta$ and $\sigma$ have the same order;

- $\theta$ is $G$-conjugate to an automorphism $\theta^{\prime} \in \operatorname{Aut}(\mathfrak{g}, \mathfrak{t}) \operatorname{such}$ that $\left.\theta^{\prime}\right|_{\mathfrak{t}}=\sigma$.

Assume that $\theta \vdash \sigma$ and that the common order $m$ of $\theta$ and $\sigma$ is nonzero in $k$. Choose a root of unity $\zeta \in k^{\times}$of order $m$, giving a grading $\mathfrak{g}=\bigoplus_{i \in \mathbb{Z} / m} \mathfrak{g}_{i}$. Recall that $\operatorname{rank}(\theta)$ is the dimension of a Cartan subspace $\mathfrak{c} \subset \mathfrak{g}_{1}$ for $\theta$. Likewise, for $\sigma \in \operatorname{Aut}(R)$, let $\operatorname{rank}(\sigma)$ be the multiplicity of $\zeta$ as a root of the characteristic polynomial of $\sigma$ on $V$. Since $\mathfrak{t}$ consists of semisimple elements, it follows that $\operatorname{rank}(\theta) \geq \operatorname{rank}(\sigma)$.

Proposition 4.1 Let $\theta \in \operatorname{Aut}(\mathfrak{g})$ be an automorphism of positive rank whose order $m$ is nonzero in $k$. Then

$$
\operatorname{rank}(\theta)=\max \{\operatorname{rank}(\sigma): \theta \vdash \sigma\}
$$

Proof: It suffices to show that there exists $\sigma \in \operatorname{Aut}(\mathfrak{g}, \mathfrak{t}) \operatorname{such}$ that $\theta \vdash \sigma$ and $\operatorname{rank}(\theta)=\operatorname{rank}(\sigma)$.

Replacing $\theta$ by a $G$-conjugate, we may assume that $\mathfrak{t}$ is the canonical Cartan subalgebra for $\theta$ (section 4.1) so that $\theta \in \operatorname{Aut}(\mathfrak{g}, \mathfrak{t})$, and $\mathfrak{c}=\mathfrak{t}_{1}$ is a Cartan subspace contained in $\mathfrak{t}$. Then $\mathfrak{c}$ is the $\zeta$-eigenspace of $\sigma:=\left.\theta\right|_{\mathfrak{t}} \in \operatorname{Aut}(R)$. Since $\theta$ has order $m$, it follows that the order of $\sigma$ divides $m$. But $\sigma$ has an eigenvalue of order $m$, so the order of $\sigma$ is exactly $m$. We therefore have $\theta \vdash \sigma$ and $\operatorname{rank}(\theta)=\operatorname{dim} \mathfrak{c}=$ $\operatorname{rank}(\sigma)$.

Given $\sigma \in \operatorname{Aut}(R)$ let $\operatorname{Kac}(\sigma)$ denote the set of normalized Kac diagrams of automorphisms $\theta \in$ $\operatorname{Aut}(\mathfrak{g}, \mathfrak{t})$ for which $\theta \vdash \sigma$. Since there are only finitely many Kac diagrams of a given order, each set $\operatorname{Kac}(\sigma)$ is finite. From Prop. 4.1 it follows that the Kac coordinates of all positive rank automorphisms of $\mathfrak{g}$ are contained in the union

$$
\bigcup_{\sigma \in \operatorname{Aut}(R) / \sim} \operatorname{Kac}(\sigma),
$$

taken over representatives of the $W$-conjugacy classes in $\operatorname{Aut}(R)$. Moreover $\operatorname{rank}(\theta)$ is the maximal $\operatorname{rank}(\sigma)$ for which the $\operatorname{Kac}$ coordinates of $\theta$ appear in $\operatorname{Kac}(\sigma)$.

\subsection{Inner automorphisms}

If $\theta \in G=\operatorname{Aut}(\mathfrak{g})^{\circ}$ is inner then its $\operatorname{Kac}$ diagram will belong to $\operatorname{Kac}(w)$ for some $w \in W$. In this section we refine the union (4) to reduce the number of classes of $w$ to consider, and we show how to compute $\operatorname{Kac}(w)$ directly from $w$, for these classes. 
A subset $J \subset\{1, \ldots, \ell\}$ is irreducible if the root system $R_{J}$ spanned by $\left\{\alpha_{j}: j \in J\right\}$ is irreducible. Two subsets $J, J^{\prime}$ are orthogonal if $R_{J}$ and $R_{J^{\prime}}$ are orthogonal.

An element $w \in W$ is $m$-admissible if $w$ has order $m$ and $w$ can be expressed as a product

$$
w=w_{1} w_{2} \cdots w_{d}
$$

where each $w_{i}$ is contained in $W_{J_{i}}$ for irreducible mutually orthogonal subsets $J_{1}, \ldots, J_{d}$ of $\{1,2, \ldots, \ell\}$ and on the reflection representation of $W_{J_{i}}$ each $w_{i}$ has an eigenvalue of order $m$ but no eigenvalue equal to 1 (so $w_{i}$ is elliptic in $W_{J_{i}}$ ). We call (5) an admissible factorization of $w$. Note that each $w_{i}$ also has order $m$, that $\operatorname{rank}(w)=\sum_{i} \operatorname{rank}\left(w_{i}\right)$, and $\operatorname{rank}\left(w_{i}\right)>0$ for $1 \leq i \leq d$.

Let $G_{i}$ be the Levi subgroup of $G$ containing $T$ and the roots from $J_{i}$, and let $G_{i}^{\prime}$ be the derived group of $G_{i}$. Each $w_{i} \in W_{J_{i}}$ has a lift $\dot{w}_{i} \in G_{i}^{\prime} \cap N$ and all such lifts are conjugate by $T \cap G_{i}$, hence the normalized Kac-coordinates of $\operatorname{Ad}\left(\dot{w}_{i}\right)$ in $\operatorname{Ad}\left(G_{i}^{\prime}\right)$ are well-defined.

Given an $m$-admissible element $w=w_{1} \cdots w_{d}$ as in (5), let $\operatorname{Kac}(w)_{\text {un }}$ be the set of un-normalized $\operatorname{Kac}$ coordinates $\left(s_{0}, s_{1}, \ldots, s_{\ell}\right)$ such that

- For $j \in J_{i}$ the coordinate $s_{j}$ is the corresponding normalized Kac coordinate of $w_{i}$ in $G_{i}^{\prime}$.

- For $i \in\{0,1, \ldots, \ell\}-J$, the coordinate $s_{i}$ ranges over a set of representatives for $\mathbb{Z} / m$.

- $\sum_{i=0}^{\ell} a_{i} s_{i}=m$.

If $w$ is any automorphism of $T$ we set $(1-w) T:=\left\{t \cdot w(t)^{-1}: t \in T\right\}$.

Lemma 4.2 If $w$ is $m$-admissible, then $\operatorname{Kac}(w)$ is the set of Kac diagrams obtained by applying the normalization algorithm of section 2.2 to the elements of $\operatorname{Kac}(w)_{\mathrm{un}}$.

Proof: Each $\operatorname{Kac}$ diagram in $\operatorname{Kac}(w)_{\text {un }}$ is that of a lift of $w$ in $N$ of order $m$. Hence the normalization of this diagram lies in $\operatorname{Kac}(w)$. Conversely, suppose $\left(s_{i}\right)$ are normalized Kac coordinates lying in $\operatorname{Kac}(w)$. By definition, there is an inner automorphism $\theta \vdash w$ (notation of section 4.2) of order $m$ with these normalized Kac-coordinates, and we may assume that $\theta=\operatorname{Ad}(n)$ for some $n \in N$, a lift of $w$. Then

$$
n=\dot{w}_{1} \dot{w}_{2} \cdots \dot{w}_{d} \cdot t
$$

where each $\dot{w}_{i}$ is a lift of $w_{i}$ and $t \in T$. Let $Z$ be the maximal torus in the center of $G_{1}^{\prime} \cdot G_{2}^{\prime} \cdots G_{d}^{\prime} \cdot T$. Then $T=Z \cdot(1-w) T$, so we may conjugate $n$ by $T$ to arrange that $t \in Z$. Next, we conjugate each $\dot{w}_{i}$ in $G_{i}^{\prime}$ to an element $t_{i} \in T \cap G_{i}^{\prime}$, thus conjugating $n$ to

$$
n^{\prime}=t_{1} \cdot t_{2} \cdots t_{d} \cdot t \in T \text {. }
$$

Since $n^{\prime}$ has order $m$ there exists $\check{\lambda} \in \check{X}$ such that $n^{\prime}=\check{\lambda}(\zeta)$. As in section 2.2 , the point $x=$ $x_{0}+\frac{1}{m} \check{\lambda} \in \mathcal{A}_{\mathbb{Q}}$ has order $m$ and the simple affine roots $\psi_{i}$ take values $\psi_{i}(x)=s_{i}^{\prime} / m$, where $s_{i}^{\prime}$ are the Kac coordinates of $n^{\prime}$ and $\sum_{i=0}^{\ell} a_{i} s_{i}^{\prime}=m$. If $j \in J_{i}$ then $s_{j}^{\prime}$ is a Kac coordinate of the $G_{i}^{\prime}$-conjugate $\dot{w}_{i}$ of $t_{i}$, and if $i \in\{0,1, \ldots, \ell\}-J$ we have $\alpha_{i}\left(n^{\prime}\right)=\zeta^{s_{i}^{\prime}}$, so the class of $s_{i}^{\prime}$ in $\mathbb{Z} / m$ is determined. Hence the Kac coordinates $\left(s_{i}^{\prime}\right)$ lie in $\operatorname{Kac}(w)_{\text {un }}$ and their normalization is $\left(s_{i}\right)$. 
Proposition 4.3 Let $\theta \in \operatorname{Aut}(\mathfrak{g})^{\circ}$ be an inner automorphism of order $m$ nonzero in $k$ with $\operatorname{rank}(\theta)>0$. Then there exists an $m$-admissible element $w \in W$ such that $\theta \vdash w$, and the rank of $\theta$ is given by

$$
\operatorname{rank}(\theta)=\max \{\operatorname{rank}(w): \theta \vdash w\}
$$

where the maximum is taken over all $W$-conjugacy classes of m-admissible elements $w \in W$ such that $\theta \vdash w$.

Proof: We may assume that $\mathfrak{t}$ is the canonical Cartan subalgebra for $\theta$, so that $\theta=\operatorname{Ad}(n)$ for some $n \in N$. The element $w=n T \in N / T=W$ has order $m$ and $\theta \vdash w$. Recall that the canonical Cartan subalgebra has the property that $\mathfrak{t}_{1}$ is a Cartan subspace for $\theta$. Hence $\operatorname{rank}(\theta)=\operatorname{rank}(w)>0$.

Assume first that $\mathfrak{t}_{0}=0$, that is, $w$ is elliptic. Then $w$ is $m$-admissible and its admissible factorization (5) is $w=w_{1}$, with $d=1$, so the proposition is proved in this case.

Assume now that $\mathfrak{t}_{0} \neq 0$. Let $R_{0}$ be the set of roots in $R$ vanishing on $\mathfrak{t}_{0}$. Since $R_{0}$ is the root system of a Levi subgroup of $G$, there is a basis $\Delta=\left\{\alpha_{1}, \alpha_{2}, \ldots, \alpha_{\ell}\right\}$ of $R$ such that $\Delta_{0}:=\Delta \cap R_{0}$ is a basis of $R_{0}$. We have $\Delta_{0}=\left\{\alpha_{j}: j \in J\right\}$ for some subset $J \subset\{1,2, \cdots, \ell\}$. Decomposing $R_{0}$ into irreducible root systems $R_{0}^{i}$, we have corresponding decompositions

$$
\begin{aligned}
R_{0} & =R_{0}^{1} \cup R_{0}^{2} \cup \cdots \cup R_{0}^{n}, \\
\Delta_{0} & =\Delta_{0}^{1} \cup \Delta_{0}^{2} \cup \cdots \cup \Delta_{0}^{n}, \\
J & =J_{1} \cup J_{2} \cup \cdots \cup J_{n}, \\
W_{J} & =W_{J_{1}} \times W_{J_{2}} \times \cdots \times W_{J_{n}}, \\
w & =w_{1} \cdot w_{2} \cdots w_{n} .
\end{aligned}
$$

By construction, $w$ is elliptic in $W_{J}$ and has an eigenvalue of order $m$ on the reflection representation of $W_{J}$. Therefore, each $w_{i}$ is elliptic in $W_{J_{i}}$ and has eigenvalues of order dividing $m$. And since $\operatorname{rank}(w)>0$ there is some number $d \geq 1$ of $w_{i}$ 's having an eigenvalue of order exactly $m$. Let the factors be numbered so that $w_{i}$ has an eigenvalue of order $m$ for $i \leq d$, and $w_{i}$ has no eigenvalue of order $m$ for $i>d$. The element

$$
w^{\prime}=w_{1} w_{2} \cdots w_{d}
$$

is $m$-admissible.

As before, let $G_{i}$ be the Levi subgroup of $G$ containing $T$ and the root subgroups from $J_{i}$, and let $G_{i}^{\prime}$ be the derived subgroup of $G_{i}$. The derived group of $C_{G}\left(\mathfrak{t}_{0}\right)$ is a commuting product $G_{1}^{\prime} \cdot G_{2}^{\prime} \cdots G_{n}^{\prime}$.

Each $w_{i}$ has a lift $\dot{w}_{i} \in N \cap G_{i}^{\prime}$; such a lift is unique up to conjugacy by $T \cap G_{i}^{\prime}$ and we have

$$
\theta=\dot{w}_{1} \dot{w}_{2} \cdots \dot{w}_{n} \cdot t
$$

for some $t \in T$. For $i>d$ we conjugate $\dot{w}_{i}$ in $G_{i}^{\prime}$ to an element $t_{i} \in T$, obtaining a conjugate $\theta^{\prime}$ of $\theta$ having the form

$$
\theta^{\prime}=\dot{w}_{1} \dot{w}_{2} \cdots \dot{w}_{d} \cdot t^{\prime}
$$

Therefore $\theta \vdash w^{\prime}$ and $w^{\prime}$ is $m$-admissible of the same rank as $\theta$. The proposition is proved. 


\section{$5 \quad$ Principal and stable gradings}

Retain the set-up of section 4 . Let $B$ be a Borel subgroup of $G=\operatorname{Aut}(\mathfrak{g})^{\circ}$ containing our fixed maximal torus $T$. The algebraic group $G$ has root datum $\left(X, R, \check{X}, \check{R}\right.$ ), where $X=X^{*}(T)$ (resp. $\check{X}=X_{*}(T)$ ) are the lattices of weights (resp. co-weights) of $T$, and $R$ (resp. $\check{R}$ ) are the sets of roots (resp. co-roots) of $T$ in $G$. The base $\Delta$ of $R$ is the set of simple roots of $T$ in $B$. As before, we choose a pinning $\left(X, R, \check{X}, \check{R},\left\{E_{i}\right\}\right)$, where $E_{i} \in \mathfrak{g}$ is a root vector for the simple root $\alpha_{i} \in \Delta$. This choice gives an isomorphism from $\operatorname{Aut}(R, \Delta)$ to the group $\Theta=\left\{\vartheta \in \operatorname{Aut}(\mathfrak{g}, \mathfrak{t}): \vartheta\left\{E_{i}\right\}=\left\{E_{i}\right\}\right\}$ of pinned automorphisms, and we have a splitting

$$
\operatorname{Aut}(\mathfrak{g})=G \rtimes \Theta
$$

\subsection{Principal gradings}

For each positive integer $m$ and pinned automorphism $\vartheta \in \operatorname{Aut}(R, \Delta)$, we have a principal grading $\mathfrak{g}=\bigoplus_{i \in \mathbb{Z} / m} \mathfrak{g}_{i}$ given (as in section 2.4) by the point $x_{m}:=\frac{1}{m} \check{\rho}+x_{0}$ (Recall that $\check{\rho}$ is the sum of the fundamental co-weights dual to the simple roots $\alpha_{i} \in \Delta$.) The normalized Kac diagram of $x_{m}$ may be obtained via the algorithm described in section 2.4. (These Kac diagrams may also be found in [8].)

Note that $\mathfrak{g}_{1}$ contains the regular nilpotent element $E:=E_{1}+E_{2}+\cdots+E_{\ell}$ associated to our pinning. If $m$ is nonzero in $k$ and we choose a root of unity $\zeta \in k^{\times}$of order $m$, then $\mathfrak{g}_{i}$ is the $\zeta^{i}$-eigenspace for the automorphism

$$
\theta_{m}:=\check{\rho}(\zeta) \vartheta .
$$

Note that the $\zeta$-eigenspace $\mathfrak{g}_{1}$ for $\theta_{m}$ contains the regular nilpotent element $E:=E_{1}+E_{2}+\cdots+E_{\ell}$ associated to our pinning. Conversely if $\theta=\check{\lambda}(\zeta) \vartheta$ is an automorphism of order $m$ whose $\mathfrak{g}_{1}$ contains a regular nilpotent element then $\theta$ is principal. If the characteristic $p$ of $k$ is zero or sufficiently large,

the element $\check{\rho}(\zeta)$ is the image of $\left[\begin{array}{ll}\zeta & 0 \\ 0 & 1\end{array}\right]$ under the principal embedding $\mathrm{PGL}_{2} \hookrightarrow G$ associated by the Jacobson-Morozov theorem to $E$. Elsewhere in the literature a principal automorphism is called "N-regular".

The first aim of this section is to show that lifts to $\operatorname{Aut}(\mathfrak{g})$ of $\mathbb{Z}$-regular elliptic automorphisms $\sigma \in$ $\operatorname{Aut}(R)$ are principal. (Recall that an automorphism $\sigma \in \operatorname{Aut}(R)$ is called elliptic if $X^{\sigma}=0$.)

More precisely, let $\sigma=w \vartheta \in W \vartheta$ be an elliptic $\mathbb{Z}$-regular automorphism of $R$ (Def. 3.1). Let $n \in N$ be a lift of $w$. Since $\sigma$ is elliptic the fixed-point group $T^{\sigma}$ is finite, so the coset $n T \vartheta \subset G \vartheta$ consists of a single $T$-orbit under conjugation. It follows that the $G$-conjugacy class $C_{\sigma}$ of $n \vartheta$ in $G \vartheta$ depends only on $\sigma$. In this section we will prove the following.

Proposition 5.1 Assume $\sigma \in W \vartheta$ is elliptic and $\mathbb{Z}$-regular and that the order $m$ of $\sigma$ is nonzero in $k$. Then the conjugacy class $C_{\sigma}$ contains $\check{\rho}(\zeta) \vartheta$ for every $\zeta \in k^{\times}$of order $m$.

The second aim of this section is to characterize the principal gradings which arise from elliptic $\mathbb{Z}$ regular automorphisms of $R$ in terms of stability (see section 5.3). 


\subsection{Conjugacy results}

If $\sigma$ is an automorphism of an abelian group $A$, we set

$$
(1-\sigma) A:=\left\{a \cdot \sigma(a)^{-1}: a \in A\right\}
$$

Let $N^{\vartheta}, W^{\vartheta}$ denote the fixed-point subgroups of $\vartheta$ in $N, W$ respectively, and let $N_{\vartheta}=\{n \in N$ : $\vartheta(n) \equiv n \bmod T\}$. It is known (see [33]) that $N_{\vartheta}=N^{\vartheta} \cdot T$. This group acts on the coset $T \vartheta$ by conjugation. Meanwhile the fixed-point group $W^{\vartheta}$ acts on the quotient torus

$$
T_{\vartheta}=T /(1-\vartheta) T
$$

whose character and cocharacter groups $X^{*}\left(T_{\vartheta}\right)=X^{\vartheta}$ and $X_{*}\left(T_{\vartheta}\right)=\check{X} /(1-\vartheta) \check{X}$ are the invariants and coinvariants of $\vartheta$ in $X$ and $\check{X}$, respectively.

We now recall some conjugacy results from [4] and [24] which are stated over $\mathbb{C}$ but whose proofs are unchanged if $\mathbb{C}$ is replaced by any algebraically closed field $k$. First, we have $[4,6.4]$ :

Lemma 5.2 The natural projection $\nu: T \rightarrow T_{\vartheta}$ induces a bijection

$$
T \vartheta / N_{\vartheta} \longrightarrow T_{\vartheta} / W^{\vartheta}
$$

sending $t \vartheta \bmod N_{\vartheta} \mapsto \nu(t) \bmod W^{\vartheta}$.

From [24, Lemma 3.2] each semisimple element $g \vartheta \in G \vartheta$ is $G$-conjugate to an element of $t \vartheta$ with $t \in T^{\vartheta}$. Now $[4,6.5]$ shows that sending $g \vartheta$ to the class of $\nu(t)$ modulo $W^{\vartheta}$ gives a bijection between the set of semisimple $G$-conjugacy classes in $G \vartheta$ and the orbit space $T_{\vartheta} / W^{\vartheta}$.

Now the affine variety $T_{\vartheta} / W^{\vartheta}$ has a canonical $\mathbb{Z}$-form, namely the ring $\mathbb{Z}\left[X^{\vartheta}\right]^{W^{\vartheta}}$ of $W^{\vartheta}$-invariants in the integral group ring of the character group $X^{\vartheta}$ of $T_{\vartheta}$. Indeed, let $X_{+}^{\vartheta}$ be the set of dominant weights in $X^{\vartheta}$ and for each $\lambda \in X_{+}^{\vartheta}$, let $\eta_{\lambda}$ be the sum in $\mathbb{Z}\left[X^{\vartheta}\right]$ over the $W^{\vartheta}$-orbit of $\lambda$, and let $\eta_{\lambda}^{k}$ be the same sum in the group ring $k\left[X^{\vartheta}\right]$. Then $\left\{\eta_{\lambda}: \lambda \in X_{+}^{\vartheta}\right\}$ and $\left\{\eta_{\lambda}^{k}: \lambda \in X_{+}^{\vartheta}\right\}$ are bases of $\mathbb{Z}\left[X^{\vartheta}\right]^{W^{\vartheta}}$ and $k\left[X^{\vartheta}\right]^{W^{\vartheta}}$ respectively, and $\left\{1 \otimes \eta_{\lambda}: \lambda \in X_{+}^{\vartheta}\right\}$ is a $k$-basis of $k \otimes_{\mathbb{Z}}\left(\mathbb{Z}\left[X^{\vartheta}\right]^{W^{\vartheta}}\right)$. It follows that the canonical mapping $\mathbb{Z}\left[X^{\vartheta}\right]^{W^{\vartheta}} \longrightarrow k\left[X^{\vartheta}\right]^{W^{\vartheta}}$ induces an isomorphism

$$
k \otimes_{\mathbb{Z}}\left(\mathbb{Z}\left[X^{\vartheta}\right]^{W^{\vartheta}}\right) \stackrel{\sim}{\longrightarrow} k\left[X^{\vartheta}\right]^{W^{\vartheta}} .
$$

The torus $T_{\vartheta}$ is a maximal torus in a connected reductive group $G_{\vartheta}$ with Weyl group $W^{\vartheta}$, so $\mathbb{Z}\left[X^{\vartheta}\right]^{W^{\vartheta}}$ has another $\mathbb{Z}$-basis, $\left\{\chi_{\lambda}: \lambda \in X_{+}^{\vartheta}\right\}$, where

$$
\chi_{\lambda}=\sum_{\mu \in X^{\vartheta}} m_{\lambda}^{\mu} \mu,
$$

and $m_{\lambda}^{\mu}$ is the multiplicity of the weight $\mu$ in the irreducible representation of highest weight $\lambda$ of the complex group with the same root datum as $G_{\vartheta}$. Therefore $k\left[X^{\vartheta}\right]^{W^{\vartheta}}$ has another $k$-basis, $\left\{\chi_{\lambda}^{k}: \lambda \in\right.$ $\left.X_{+}^{\vartheta}\right\}$, where $\chi_{\lambda}^{k} \in k\left[X^{\vartheta}\right]^{W^{\vartheta}}$ is the image of $1 \otimes \chi_{\lambda}$ under the isomorphism (6). 
We now regard $G$ as a Chevalley group scheme over $\mathbb{Z}$, writing $G(A)$ for the group of $A$-valued points in a commutative ring $A$. The group heretofore denoted by $G$ is now $G(k)$. Likewise $T$ and $N$ are now group schemes over $\mathbb{Z}$.

Let $\lambda \in X_{+}^{\vartheta}$ and let $V$ be the irreducible representation of $G(\mathbb{C})$ of highest weight $\lambda$. Since $\vartheta \lambda=\lambda$ it follows that $V$ extends uniquely to a representation of $G(\mathbb{C}) \cdot\langle\vartheta\rangle$ such that $\vartheta$ acts trivially on the highest weight space $V(\lambda)$.

Choose a $G(\mathbb{Z})$-stable lattice $M$ in $V$ such that $M \cap V(\mu)$ spans each weight space $V(\mu)$ in $V$ and $\vartheta M=M$. For example, we could take $M$ to be the admissible $\mathbb{Z}$-form of $V$ constructed by Kostant in [15]. We get a representation of $G(k) \cdot\langle\vartheta\rangle$ on $V_{k}:=k \otimes M$ which may be reducible and which depends on $M$. However, since $M$ contains a basis of $V$, the traces on $V_{k}$ of elements of $G(k) \cdot\langle\vartheta\rangle$ are independent of the choice of $M$.

Let $A=\mathbb{Z}[\zeta] \subset \mathbb{C}$ be the cyclotomic ring generated by a root of unity $\zeta \in \mathbb{C}^{\times}$of order $m$. Assume that $k$ is algebraically closed and $m$ is nonzero in $k$. Choose $\zeta_{k} \in k^{\times}$a root of unity of order $m$. We have ring homomorphisms

$$
\mathbb{C} \stackrel{\iota}{\longleftarrow} A \stackrel{\pi}{\longrightarrow} k,
$$

where $\iota$ is the inclusion and $\pi(\zeta)=\zeta_{k}$. We use the same letters to denote maps on groups of points, e.g.,

$$
G(\mathbb{C}) \stackrel{\iota}{\leftrightarrows} G(A) \stackrel{\pi}{\longrightarrow} G(k)
$$

and similarly for $T$ and $N$.

Lemma 5.3 Let $s, t \in T(k)^{\vartheta}$ be elements of order $m$ such that $\operatorname{tr}\left(s \vartheta, V_{k}\right)=\operatorname{tr}\left(t \vartheta, V_{k}\right)$ for all irre-

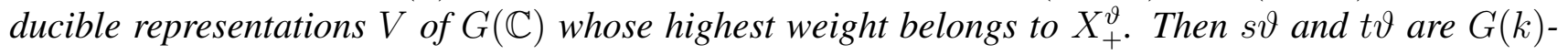
conjugate.

Proof: Let $V^{\prime}$ be the representation of $G_{\vartheta}(\mathbb{C})$ with the same highest weight as $V$. And choose a lattice $M^{\prime} \subset V^{\prime}$ analogous to $M$ above. Since $s$ has order $m$ there is a co-weight $\check{\omega} \in \check{X}$ such that

$$
s=\check{\omega}\left(\zeta_{k}\right)=\pi \check{\omega}(\zeta)
$$

For each $\mu \in X^{\vartheta}$ let $M(\mu)=M \cap V(\mu)$ and likewise set $M^{\prime}(\mu)=M^{\prime} \cap V^{\prime}(\mu)$. We have

$$
\begin{aligned}
\operatorname{tr}\left(s \vartheta, V_{k}\right) & =\sum_{\mu \in X_{\vartheta}} \mu(s) \cdot \operatorname{tr}(\vartheta, k \otimes M(\mu))=\sum_{\mu \in X^{\vartheta}} \zeta_{k}^{\langle\mu, \breve{\omega}\rangle} \cdot \pi(\operatorname{tr}(\vartheta, M(\mu))) \\
& =\pi\left(\sum_{\mu \in X_{\vartheta}} \zeta^{\langle\mu, \check{\omega}\rangle} \cdot \operatorname{tr}(\vartheta, M(\mu))\right)
\end{aligned}
$$

By a result of Jantzen (see for example [17]) we have

$$
\sum_{\mu \in X^{\vartheta}} \zeta^{\langle\mu, \breve{\omega}\rangle} \cdot \operatorname{tr}(\vartheta, M(\mu))=\sum_{\mu \in X^{\vartheta}} \zeta^{\langle\mu, \check{\omega}\rangle} \cdot \operatorname{dim} M^{\prime}(\mu) .
$$


It follows that

$$
\operatorname{tr}\left(s \vartheta, V_{k}\right)=\pi\left(\sum_{\mu \in X^{\vartheta}} \zeta^{\langle\mu, \check{\omega}\rangle} \cdot \operatorname{dim} M^{\prime}(\mu)\right)=\operatorname{tr}\left(\nu(s), V_{k}^{\prime}\right) .
$$

Applying this identity to $t \vartheta$ as well, we find that

$$
\operatorname{tr}\left(\nu(s), V_{k}^{\prime}\right)=\operatorname{tr}\left(\nu(t), V_{k}^{\prime}\right)
$$

Therefore $\chi_{\lambda}^{k}(\nu(s))=\chi_{\lambda}^{k}(\nu(t))$ for every $\lambda \in X_{+}^{\vartheta}$. Since these $\chi_{\lambda}^{k}$ are a basis of $k\left[X^{\vartheta}\right]^{W^{\vartheta}}$, it follows from [31, Cor. 6.6] that $\nu(s) \equiv \nu(t) \bmod W^{\vartheta}$. By Lemma 5.2 we have that $s \vartheta$ and $t \vartheta$ are $G(k)$ conjugate, as claimed.

Now suppose $g \in G(\mathbb{Z})$ and $g \vartheta$ is semisimple of order $m$. Let $s \in T(\mathbb{C})^{\vartheta}$ and $t \in T(k)^{\vartheta}$ be such that $\iota(g) \vartheta$ is $G(\mathbb{C})$-conjugate to $s \vartheta$ and $\pi(g) \vartheta$ is $G(k)$-conjugate to $t \vartheta$.

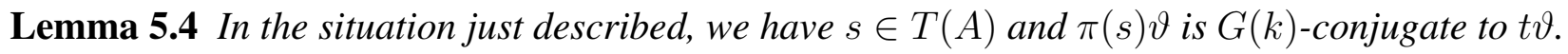

Proof: As above we have $s=\check{\omega}(\zeta)$ for some co-weight $\check{\omega} \in \check{X}$. It follows that $s \in T(A)$. Moreover, $g \vartheta$ preserves the lattice $M$, so we have

$$
\operatorname{tr}(\iota(g) \vartheta, M)=\operatorname{tr}(s \vartheta, V)=\sum_{\mu \in X^{\vartheta}} \zeta^{\langle\mu, \check{\omega}\rangle} \cdot \operatorname{tr}(\vartheta, M(\mu)) .
$$

Applying $\pi$ to both sides we get

$$
\pi(\operatorname{tr}(\iota(g) \vartheta, M))=\sum_{\mu \in X^{\vartheta}} \zeta_{k}^{\langle\mu, \breve{\omega}\rangle} \cdot \operatorname{tr}\left(\vartheta, V_{k}(\mu)\right)=\operatorname{tr}\left(\pi(s) \vartheta, V_{k}\right) .
$$

On the other hand, we can first apply $\pi: G(A) \rightarrow G(k)$ and then take traces. This gives

$$
\pi(\operatorname{tr}(\iota(g) \vartheta, M))=\operatorname{tr}\left(\pi(g) \vartheta, V_{k}\right)=\operatorname{tr}\left(t \vartheta, V_{k}\right) .
$$

Comparing the expressions (7) and (8) and using Lemma 5.3 we see that $\pi(s) \vartheta$ and $t \vartheta$ are $G(k)$ conjugate as claimed.

We are ready to prove Prop. 5.1. Recall that $w \vartheta \in W \vartheta$ is an elliptic $\mathbb{Z}$-regular automorphism of $R$ whose order $m$ is nonzero in the algebraically closed field $k$. Let $\zeta \in k^{\times}$be a root of unity of order $m$. Recall that $\check{\rho}$ is the sum of the fundamental co-weights arising from our chosen pinning. We have $\check{\rho} \in \check{X}^{\vartheta}$ and $\check{\rho}(\zeta) \in T(k)^{\vartheta}$. We now prove Prop. 5.1 in the following form.

Proposition 5.5 For any lift $n \in N(k)$ of $w$, the element $n \vartheta \in G(k) \vartheta$ is $G(k)$-conjugate to $\check{\rho}(\zeta) \vartheta$.

Proof: Assume first that $k$ has characteristic zero. In this case the proof relies on [23, Thm. 3.3] and is similar to the proof of [23, Thm. 4.2 (iii)]. The automorphism $\tau:=\check{\rho}(\zeta) \vartheta \in \operatorname{Aut}(\mathfrak{g})$ has order $m$ and gives a grading $\mathfrak{g}=\oplus_{i \in \mathbb{Z} / m} \mathfrak{g}_{i}^{\prime}$, where $\mathfrak{g}_{i}^{\prime}$ is the $\zeta^{i}$-eigenspace of $\tau$. The sum $E=\sum_{i=1}^{\ell} E_{i}$ of the simple root vectors in our pinning belongs to $\mathfrak{g}_{1}^{\prime}$. By [23, Thm. 3.3(v)], the dimension of a Cartan subspace $\mathfrak{c} \subset \mathfrak{g}_{1}^{\prime}$ may be computed as follows. Let $f_{1}, \ldots, f_{\ell} \in k[\mathfrak{t}]$ be homogeneous generators for 
the algebra of $W$-invariant polynomials on $\mathfrak{t}$. Assume, as we may, that each $f_{i}$ is an eigenvector for $\vartheta$, with eigenvalue denoted $\varepsilon_{i}$, and set $d_{i}=\operatorname{deg} f_{i}$. The integer

$$
a(m, \vartheta):=\left|\left\{i: 1 \leq i \leq \ell, \varepsilon_{i} \zeta^{d_{i}}=1\right\}\right|
$$

depends only on $m$ and $\vartheta$, and we have

$$
\operatorname{dim} \mathfrak{c}=a(m, \vartheta)
$$

Let $\mathfrak{s}$ be a canonical Cartan subalgebra for $\tau$ (section 4.1). There exists $g \in G$ such that $\mathfrak{t}=\operatorname{Ad}(g) \mathfrak{s}$, and we set $\theta^{\prime}=g \tau g^{-1}$. Since $\theta^{\prime}$ normalizes $\mathfrak{t}$ and belongs to $G \vartheta$ we have $\theta^{\prime} \in N \vartheta$. Let $w^{\prime} \vartheta \in W \vartheta$ be the projection of $\theta^{\prime}$. Then $\operatorname{Ad}(g) \mathfrak{c}$ is the $\zeta$-eigenspace $\mathfrak{t}\left(w^{\prime} \vartheta, \zeta\right)$ of $w^{\prime} \vartheta$ in $\mathfrak{t}$, so

$$
\operatorname{dim} \mathfrak{t}\left(w^{\prime} \vartheta, \zeta\right)=a(m, \vartheta)
$$

Since $w \vartheta$ is $\mathbb{Z}$-regular and therefore $k$-regular (by Prop. 3.2), it follows from [29, Prop. 3.6] that we also have $\operatorname{dim} \mathfrak{t}(w \vartheta, \zeta)=a(m, \vartheta)$, and therefore

$$
\operatorname{dim} \mathfrak{t}(w \vartheta, \zeta)=\operatorname{dim} \mathfrak{t}\left(w^{\prime} \vartheta, \zeta\right)
$$

By [29, Thm. 6.4 (iv)] the elements $w \vartheta, w^{\prime} \vartheta \in W \vartheta$ are conjugate under $W$. It follows that $n \vartheta$ is $N$-conjugate to an element of $T \theta^{\prime}$. As $w^{\prime} \vartheta$ is also elliptic, it follows that $n \vartheta$ is actually conjugate to $\theta^{\prime}$, and hence to $\tau=\check{\rho}(\zeta) \vartheta$, as claimed.

Now assume that $k$ has positive characteristic not dividing $m$. Let $A$ be the cyclotomic subring of $\mathbb{C}$ generated by $z=e^{2 \pi i / m}$ and let $\pi: A \rightarrow k$ be the ring homomorphism mapping $z \mapsto \zeta$. By ellipticity, all lifts of $w \vartheta$ to $N(k) \vartheta$ are $T(k)$-conjugate, so we may choose our lift to be of the form $\pi(n)$ with $n \in N(\mathbb{Z})$. From the characteristic zero case just proved, we have that $\iota(n) \vartheta$ is $G(\mathbb{C})$-conjugate to $\check{\rho}(z) \vartheta$. By Lemma 5.4 it follows that $\pi(n) \vartheta$ is $G(k)$-conjugate to $\check{\rho}(\zeta) \vartheta$, as claimed.

\subsection{Stable gradings}

Let $H$ be a connected reductive $k$-group acting on a $k$-vector space $V$. A vector $v \in V$ is called $H$-stable (in the sense of Geometric Invariant Theory, see [22]) if the $H$-orbit of $v$ is closed and the stabilizer of $v$ in $H$ is finite. The second condition means that the stabilizer $H_{v}$ is a finite algebraic group: it has only finitely many points over the algebraically closed field $k$.

Recall we are assuming the characteristic of $k$ is not a torsion prime for $G$ and that the period $m$ of the grading $\mathfrak{g}=\oplus_{i \in \mathbb{Z} / m} \mathfrak{g}_{i}$ is nonzero in $k$. We have chosen a root of unity $\zeta \in k^{\times}$of order $m$, and $\theta \in \operatorname{Aut}(\mathfrak{g})$ is the automorphism of order $m$ whose $\zeta^{i}$-eigenspace is $\mathfrak{g}_{i}$.

We say the grading $\mathfrak{g}=\oplus_{i \in \mathbb{Z} / m} \mathfrak{g}_{i}$ (or the automorphism $\theta$ ) is stable if there are $G_{0}$-stable vectors in $\mathfrak{g}_{1}$. In this section we will show that stable gradings are closely related to elliptic $\mathbb{Z}$-regular automorphisms of the root system $R$.

Lemma 5.6 $A$ vector $v \in \mathfrak{g}_{1}$ is stable if and only if $v$ is a regular semisimple element of $\mathfrak{g}$ and the action of $\theta$ on the Cartan subalgebra centralizing $v$ is elliptic. 
Proof: Vinberg showed ([35, Prop. 3]) that the $G_{0}$-orbit of $v$ is closed in $\mathfrak{g}_{1}$ if and only if $v$ is semisimple in $\mathfrak{g}$. His proof works also in positive characteristic (see [19, 2.12-3]). If $v$ is semisimple its centralizer $C_{G}(v)$ is connected (since $p$ is not a torsion prime, by [34, Thm. 3.14]) and reductive with semisimple derived subgroup $H[5,13.19,14.2]$. As $v$ is an eigenvector for $\theta$ we have $\theta(H)=H$. If $v$ is stable then $H^{\theta}$ is finite. Let $\pi: H_{s c} \rightarrow H$ be the simply-connected covering of $H$. We lift $\theta$ to an automorphism of $H_{s c}$, denoting it again by $\theta$. Now $H_{s c}^{\theta}$ is connected [33, chap. 8] so $\pi\left(H_{s c}^{\theta}\right) \subset\left(H^{\theta}\right)^{\circ}$ is trivial. Since $\operatorname{ker} \pi$ is finite, we must have $H_{s c}^{\theta}=1$. This implies that $H_{s c}=1$. For otherwise, by [33, chap. 8], there would be a maximal torus $T^{\prime}$ contained in a Borel subgroup $B^{\prime}$ of $H_{s c}$ such that $\theta\left(T^{\prime}\right)=T^{\prime}$ and $\theta\left(B^{\prime}\right)=B^{\prime}$, and $H_{s c}^{\theta}$ would have rank equal to the number of $\theta$-orbits on the set of simple roots of $T^{\prime}$ in $B^{\prime}$. Therefore $H_{s c}=1$, so $H=1$ and $C_{G}(v)$ is a torus. This means that $v$ is regular in $\mathfrak{g}$. The reverse implication is clear.

Prop. 5.1 and Lemma 5.6 have the following corollaries.

Corollary 5.7 Let $\theta \in G \vartheta$ have order $m$ nonzero in $k$. The following are equivalent.

1. The grading on $\mathfrak{g}$ given by $\theta$ is stable;

2. The action of $\theta$ on its canonical Cartan subalgebra induces an elliptic $\mathbb{Z}$-regular automorphism of $R$;

3. $\theta$ is principal and $m$ is the order of an elliptic $\mathbb{Z}$-regular element of $W \vartheta$.

Corollary 5.8 The map sending a stable automorphism $\theta \in \operatorname{Aut}(\mathfrak{g})$ to the automorphism of $R$ induced by the action of $\theta$ on its canonical Cartan subalgebra gives a bijection between the G-conjugacy classes of stable automorphisms of $\mathfrak{g}$ and the $W$-conjugacy classes of elliptic $\mathbb{Z}$-regular automorphisms of $R$.

\section{Affine-pinned automorphisms}

In this section we construct certain automorphisms of $\mathfrak{g}$ arising from symmetries of the affine Dynkin diagram. These will be used to study outer automorphisms of $E_{6}$.

Assume $\mathfrak{g}$ is a simple Lie algebra over $\mathbb{C}$ with adjoint group $G=\operatorname{Aut}(\mathfrak{g})^{\circ}$. Let $N, T$ be the normalizer and centralizer of a Cartan subalgebra $\mathfrak{t}$ of $\mathfrak{g}$ and let $W=N / T$. Let $R$ be the set of roots of $T$ in $\mathfrak{g}$ and choose a base $\Delta=\left\{\alpha_{1}, \ldots, \alpha_{\ell}\right\}$ of $R$. Let $\alpha_{0}$ be the lowest root of $R$ with respect to $\Delta$ and set $\Pi=\left\{\alpha_{i}: i \in I\right\}$, where $I=\{0,1, \ldots, \ell\}$. The subgroup of $W$ preserving $\Pi$,

$$
W_{\Pi}=\{w \in W: w \Pi=\Pi\}
$$

is isomorphic to the fundamental group of $G$. Each element $w \in W_{\Pi}$ determines a permutation $\sigma$ of $I$ such that

$$
w \cdot \alpha_{i}=\alpha_{\sigma(i)}
$$

Choose a Chevalley lattice $\mathfrak{g}_{\mathbb{Z}} \subset \mathfrak{g}$ spanned by a lattice in $\mathfrak{t}$ and root vectors for $T$. An affine pinning is a set $\widetilde{\Pi}=\left\{E_{0}, E_{1}, \cdots, E_{\ell}\right\}$ consisting of nonzero root vectors $E_{i} \in \mathfrak{g}_{\alpha_{i}} \cap \mathfrak{g}(\mathbb{Z})$ for each $i \in I$. Let 
$N(\mathbb{Z})$ be the stabilizer of $\mathfrak{g}(\mathbb{Z})$ in $N$, and consider the subgroup

$$
N_{\widetilde{\Pi}}=\{n \in N(\mathbb{Z}): n \widetilde{\Pi}=\widetilde{\Pi}\} .
$$

Lemma 6.1 Let $\widetilde{\Pi}$ be an affine pinning. Then the projection $N \rightarrow W$ restricts to an isomorphism $f: N_{\widetilde{\Pi}} \stackrel{\sim}{\longrightarrow} W_{\Pi}$.

Proof: It is clear that $f\left(N_{\widetilde{\Pi}}\right) \subset W_{\Pi}$. An element in ker $f$ lies in $T$ and fixes each root vector $E_{i}$, hence lies in the center of $G$, which is trivial since $G$ is adjoint. Hence $f$ is injective.

Let $w \in W_{\Pi}$. Since the projection $N \rightarrow W$ is surjective on $N(\mathbb{Z})$ [32, Lemma 22], there is a lift $n^{\prime}$ of $w$ such that $n^{\prime} \in N(\mathbb{Z})$. For each $i \in I$ we have $n^{\prime} \cdot E_{i}=c_{i} E_{\sigma(i)}$, for some $c_{i}= \pm 1$.

Let $\check{\omega}_{1}, \ldots, \check{\omega}_{\ell} \in X_{*}(T)$ be the fundamental coweights of $T$ dual to $\alpha_{1}, \ldots, \alpha_{\ell}$. The element $t=$ $\prod_{i=1}^{\ell} \check{\omega}_{i}\left(c_{i}\right)$ lies in $T(\mathbb{Z})$ and the new lift $n=n^{\prime} t$ of $w$ satisfies $n \cdot E_{i}=E_{\sigma(i)}$ for $1 \leq i \leq \ell$.

Let $d$ be the order of $w$. Then $\sigma^{d}=1$ so $n^{d}$ fixes $E_{i}$ for each $1 \leq i \leq \ell$. Hence $n^{d} \in T$ and belongs to the kernel of each simple root $\alpha_{i}$. Since $G$ is adjoint, it follows that $n^{d}=1$.

Let $i=\sigma(0)$. It follows from [6, VI.2.2] that $\sigma^{j}(0) \neq 0$ for $1 \leq j<d$. By what has been proved, we have

$$
n^{-1} \cdot E_{i}=n^{d-1} \cdot E_{i}=E_{\sigma^{d-1}(i)}=E_{\sigma^{-1}(i)}=E_{0} .
$$

It follows that $n \cdot E_{0}=E_{i}$, so $n$ is a lift of $w$ in $N_{\widetilde{\Pi}}$.

Now let $k$ be an algebraically closed field of characteristic not equal to two, and view $G$ as a group scheme over $\mathbb{Z}$, via the lattice $\mathfrak{g}_{\mathbb{Z}}$. Take $w \in W_{\Pi}$ of order two. Again from [6, VI.2.2] there exists a unique minuscule coweight $\check{\omega}_{j}$ such that $w \check{\omega}_{j}=-\check{\omega}_{j}$. Since $2 \neq 0$ in $k$, the natural map $T(\mathbb{Z}) \rightarrow T(k)$ is injective, which implies that the map $N(\mathbb{Z}) \rightarrow N(k)$ is injective. We now let $n$ be the image in $N(k)$ of the unique lift of $w$ in $N_{\widetilde{\Pi}}$.

Proposition 6.2 There exists an affine pinning $\widetilde{\Pi}$ such that $n$ is $G(k)$-conjugate to $\check{\omega}_{j}(-1)$. The Kac coordinates of $\operatorname{Ad}(n)$ are given by:

$$
s_{i}=\left\{\begin{array}{lll}
1 & \text { for } & i \in\{0, j\} \\
0 & \text { for } & i \notin\{0, j\} .
\end{array}\right.
$$

These labels give the unique w-invariant Kac-diagram of order two having $s_{0} \neq 0$.

Proof: By [7, Lemma 5] there are mutually orthogonal roots $\gamma_{1}, \ldots, \gamma_{m} \in R$ with corresponding reflections $r_{1}, \ldots, r_{m} \in W$, such that

$$
w=r_{1} r_{2} \cdots r_{m} .
$$

Since $\check{\omega}_{j}$ is minuscule we have $\left\langle\alpha, \check{\omega}_{j}\right\rangle \in\{-1,0,1\}$ for each $\alpha \in R$. The positive roots made negative by $w$ are those for which $\left\langle\alpha, \check{\omega}_{j}\right\rangle \neq 0$. Since $w \gamma_{i}=-\gamma_{i}$ for each $i$, we may choose the sign of each $\gamma_{i}$ so that $\left\langle\gamma_{i}, \check{\omega}_{j}\right\rangle=1$. And since

$$
-\check{\omega}_{j}=w \cdot \check{\omega}_{j}=\check{\omega}_{j}-\sum_{i=1}^{m}\left\langle\gamma_{i}, \check{\omega}_{j}\right\rangle \check{\gamma}_{i},
$$


it then follows that

$$
\check{\gamma}_{1}+\check{\gamma}_{2}+\cdots+\check{\gamma}_{m}=2 \check{\omega}_{j}
$$

For each $i=1, \ldots, m$ there exists a morphism $\varphi_{i}: S L_{2} \rightarrow G$ over $\mathbb{Z}$ whose restriction to the diagonal subgroup is given by

$$
\varphi_{i}\left(\left[\begin{array}{cc}
t & 0 \\
0 & t^{-1}
\end{array}\right]\right)=\check{\gamma}_{i}(t)
$$

and such that $\varphi_{i}\left(\left[\begin{array}{cc}0 & -1 \\ 1 & 0\end{array}\right]\right) \in N(\mathbb{Z})$ and is a representative of $r_{i}$.

Since the roots $\gamma_{i}$ are mutually orthogonal, the images of these homomorphisms $\varphi_{i}$ commute with one another. Hence we have a $\mathbb{Z}$-morphism

$$
\varphi: S L_{2} \rightarrow G, \quad \text { given by } \quad \varphi\left(\left[\begin{array}{ll}
a & b \\
c & d
\end{array}\right]\right)=\prod_{i=1}^{m} \varphi_{i}\left(\left[\begin{array}{ll}
a & b \\
c & d
\end{array}\right]\right) .
$$

By equation (9) the element

$$
n:=\varphi\left(\left[\begin{array}{cc}
0 & -1 \\
1 & 0
\end{array}\right]\right)
$$

belongs to $N(\mathbb{Z})$ and represents $w$. Equation (10) implies that

$$
\varphi\left(\left[\begin{array}{cc}
t & 0 \\
0 & t^{-1}
\end{array}\right]\right)=\check{\omega}_{j}(t)^{2}
$$

which in turn implies that $n$ has order two. Since the matrices $\left[\begin{array}{cc}0 & -1 \\ 1 & 0\end{array}\right]$ and $\left[\begin{array}{cc}\sqrt{-1} & 0 \\ 0 & -\sqrt{-1}\end{array}\right]$ are conjugate in $\mathrm{SL}_{2}$, it follows that $n$ is conjugate to $\check{\omega}_{j}(-1)$ in $G$, and that $\operatorname{Ad}(n)$ has the asserted $\operatorname{Kac}-$ coordinates.

We construct an affine pinning stable under $n$ as follows. Choose representatives $\alpha_{i}$ of the $w$-orbits in $\Pi$, and choose arbitrary nonzero root vectors $E_{i} \in \mathfrak{g}(\mathbb{Z})$ for these roots. Let $\sigma$ be the permutation of $I$ induced by $w$. If $w \cdot \alpha_{i} \neq \alpha_{i}$, let $E_{\sigma(i)}=n \cdot E_{i}$. Since $n$ has order two, we have $n \cdot E_{\sigma(i)}=E_{i}$. If $w \cdot \alpha_{i}=\alpha_{i}$ then $\alpha_{i}$ is orthogonal to each of the roots $\gamma_{1}, \ldots, \gamma_{m}$, since the latter are negated by $w$. It follows that the image of each homomorphism $\varphi_{1}, \ldots, \varphi_{m}$ centralizes the root space $\mathfrak{g}_{\alpha_{i}}$, so any nonzero vector $E_{i} \in \mathfrak{g}_{\alpha_{s}} \cap \mathfrak{g}(\mathbb{Z})$ is fixed by $n$. The collection $\widetilde{\Pi}=\left\{E_{i}\right\}$ of vectors thus defined is an affine pinning stable under $n$.

The following lemma will also be useful.

Lemma 6.3 Let $S=\left(T^{n}\right)^{\circ}$ be the identity component of the subgroup of $T$ centralized by $n$. Then $S$ is centralized by the entire group $\varphi\left(\mathrm{SL}_{2}\right)$.

Proof: Since $2 \check{\omega}_{j}$ is a simple co-weight in $\varphi\left(\mathrm{SL}_{2}\right)$ and $\check{\omega}_{j}$ is minuscule, we have that $\left\langle\alpha, 2 \check{\omega}_{j}\right\rangle \in$ $\{-2,0,2\}$ for every root $\alpha \in R$. Hence $\varphi\left(\mathrm{SL}_{2}\right)$ acts on $\mathfrak{g}$ as a sum of copies of the trivial and adjoint representations. Applying the element

$$
\left[\begin{array}{cc}
1 & 0 \\
-t & 1
\end{array}\right] \cdot\left[\begin{array}{cc}
0 & -1 / t \\
t & 0
\end{array}\right]=\left[\begin{array}{cc}
1 & -1 / t \\
0 & 1
\end{array}\right] \cdot\left[\begin{array}{cc}
1 & 0 \\
t & 1
\end{array}\right]
$$


to a vector in the zero weight space and comparing components in the -2 weight space, we find (since the characteristic of $k$ is not two) that any vector in $\mathfrak{g}$ invariant under the normalizer of $2 \check{\omega}_{j}\left(k^{\times}\right)$in $\varphi\left(\mathrm{SL}_{2}\right)$ is invariant under all of $\varphi\left(\mathrm{SL}_{2}\right)$. Since the Lie algebra of $S$ consists of such vectors, the lemma is proved.

\section{Little Weyl groups}

Let $\theta$ be an automorphism of $\mathfrak{g}$ whose order $m$ is invertible in $k$. Choose a root of unity $\zeta \in k^{\times}$of order $m$ and let $\mathfrak{g}=\oplus_{i \in \mathbb{Z} / m} \mathfrak{g}_{i}$ be the grading of $\mathfrak{g}$ into $\zeta^{i}$-eigenspaces of $\theta$. Choose a Cartan subspace $\mathfrak{c}$ in $\mathfrak{g}_{1}$ and assume the rank $r=\operatorname{dim} \mathfrak{c}$ is positive. The little Weyl group is defined as

$$
W(\mathfrak{c}, \theta)=N_{G_{0}}(\mathfrak{c}) / Z_{G_{0}}(\mathfrak{c})
$$

where $G_{0}=\left(G^{\theta}\right)^{\circ}$ is the connected subgroup of $G$ with Lie algebra $\mathfrak{g}_{0}$. When it is necessary to specify $G$ in the little Weyl group we will write $W_{G}(\mathfrak{c}, \theta)$.

It is clear from the definition that $W(\mathfrak{c}, \theta)$ acts faithfully on $\mathfrak{c}$. From [35] and [19], it is known that the action of $W(\mathfrak{c}, \theta)$ on $\mathfrak{c}$ is generated by transformations fixing a hyperplane in $\mathfrak{c}$, that the restriction map $k\left[\mathfrak{g}_{1}\right]^{G_{0}} \rightarrow k[\mathfrak{c}]^{W(\mathfrak{c}, \theta)}$ is an isomorphism, and that this ring is a polynomial ring with homogeneous generators $f_{1}, \ldots, f_{r}$, such that

$$
|W(\mathfrak{c}, \theta)|=\prod_{i=1}^{r} \operatorname{deg}\left(f_{i}\right)
$$

\subsection{Upper bounds on the little Weyl group}

Recall we have fixed a Cartan subalgebra $\mathfrak{t}$ in $\mathfrak{g}$, with normalizer and centralizer $N$ and $T$ in $G$ and we have identified $W=N / T$.

Replacing $\theta$ by a $G$-conjugate if necessary, we may assume $\mathfrak{t}$ is the canonical Cartan subalgebra for $\theta$ (see 4.1). In particular $\mathfrak{c}$ is the $\zeta$-eigenspace of $\theta$ in $\mathfrak{t}$. Then $\theta$ normalizes $N$ and $T$ in $\operatorname{Aut}(\mathfrak{g})$, giving an action of $\theta$ on $W$; let $W^{\theta}=\{y \in W: \theta(y)=y\}$ be the fixed point subgroup of $\theta$ in $W$.

Elements in $W^{\theta}$ commute with the action of $\theta$ on $\mathfrak{t}$, so $W^{\theta}$ acts on the eigenspace $\mathfrak{c}$. Let

$$
W_{1}^{\theta}:=W^{\theta} / C_{W}(\mathfrak{c})^{\theta}
$$

be the quotient acting faithfully on $\mathfrak{c}$. Since $\mathfrak{t}$ is a Cartan subalgebra in the Levi subalgebra $\mathfrak{m}=\mathfrak{z}_{\mathfrak{g}}(\mathfrak{c})$, it follows that every element of $W(\mathfrak{c}, \theta)$ has a representative in $N$ and that $W(\mathfrak{c}, \theta)$ may be viewed as a subgroup of $W_{1}^{\theta}$. Thus, we have an embedding

$$
W(\mathfrak{c}, \theta) \hookrightarrow W_{1}^{\theta}
$$

Note that $W(\mathfrak{c}, \theta)$ is more subtle than $W_{1}^{\theta}$. For it can happen that two automorphisms $\theta$ and $\theta^{\prime}$ of the same order agree on $\mathfrak{t}$ and $W$, so they have the same Cartan subspace $\mathfrak{c}$ and $W_{1}^{\theta}=W_{1}^{\theta^{\prime}}$, but nevertheless 
$W(\theta, \mathfrak{c}) \neq W\left(\theta^{\prime}, \mathfrak{c}\right)$ (e.g. cases $4_{a}$ and $4_{b}$ in $E_{6}$; these examples are also used in $[23,4.5]$ to illustrate other subtleties).

A still coarser group, depending only on $\mathfrak{c}$ and not on $\theta$ is

$$
W(\mathfrak{c}):=N_{W}(\mathfrak{c}) / C_{W}(\mathfrak{c}) .
$$

As subgroups of $\mathrm{GL}(\mathfrak{c})$, we have containments

$$
W(\mathfrak{c}, \theta) \subset W_{1}^{\theta} \subset W(\mathfrak{c}) .
$$

Under certain circumstances one or both of these containments is an equality.

Lemma 7.1 Suppose $\mathfrak{c}$ contains a regular element of $\mathfrak{g}$. Then

$$
W_{1}^{\theta}=W^{\theta}=W(\mathfrak{c})
$$

Proof: By regularity it is clear that $W_{1}^{\theta}=W^{\theta}$ and that $W(\mathfrak{c})=N_{W}(\mathfrak{c})$. And any $y \in N_{W}(\mathfrak{c})$ commutes with the scalar action of $\theta$ on $\mathfrak{c}$ so the commutator $[y, \theta]$ is trivial in $W$, again by regularity.

Panyushev [23, Thm. 4.7] has shown that both containments above are equalities if $\theta$ is principal:

Proposition 7.2 (Panyushev) If $\theta$ is principal then $W(\mathfrak{c}, \theta)=W_{1}^{\theta}=W(\mathfrak{c})$.

We note that Panyushev works in characteristic zero, but his geometric proof works equally well in good characteristic $p \nmid m$, using the invariant theoretic results of [19].

Corollary 7.3 If $\theta$ is principal and the restriction of $\theta$ to $\mathfrak{t}$ induces a $\mathbb{Z}$-regular automorphism of $R$ then $W(\mathfrak{c}, \theta)=W^{\theta}$.

Proof: By Prop. 3.2, $\mathbb{Z}$-regularity implies $k$-regularity, so $W(\mathfrak{c})=W_{1}^{\theta}$ is just $W^{\theta}$.

This sharpens the first result in this direction, which was proved in Vinberg's original work [35, Prop. 19]:

Corollary 7.4 (Vinberg) If $\theta$ gives a stable grading on $\mathfrak{g}$ then $W(\mathfrak{c}, \theta)=W^{\theta}$.

\subsection{Little Weyl groups for inner gradings}

Assume now that $\theta$ is inner, and let the restriction of $\theta$ to $\mathfrak{t}$ be given by the element $w \in W$. In this section we give upper and lower bounds for $W(\mathfrak{c}, \theta)$ depending only on $w$, under certain conditions; these will suffice to compute almost all little Weyl groups in type $E_{n}$. The fixed-point group

$$
W^{\theta}=C_{W}(w),
$$

is now the centralizer of $w$ in $W$, which acts on the $\zeta$-eigenspace $\mathfrak{c}$ of $w$ in $\mathfrak{t}$. The quotient by the kernel of this action is the group $W_{1}^{\theta}$. Simple upper and lower bounds for $W(\mathfrak{c}, \theta)$ can be obtained as follows. 
Lemma 7.5 If $U$ is any subgroup of $C_{W}(w)$ acting trivially on $\mathfrak{c}$ then we have the inequalities

$$
m \leq|W(\mathfrak{c}, \theta)| \leq \frac{\left|C_{W}(w)\right|}{|U|} .
$$

Proof: Since $\theta$ is semisimple it lies in the identity component $G_{0}$ of its centralizer in $G$. Hence the cyclic group $\langle\theta\rangle$ embeds in $W(\mathfrak{c}, \theta)$, whence the lower bound. The upper bound follows from (12).

Information about $C_{W}(w)$, including its order, is given in [7]. Using the tables therein, one can often find a fairly large subgroup $U \subset C_{W}(w)$ as in Lemma 7.5.

Example 1: In type $E_{8}$ there are eight cases (namely $12_{b}$ through $12_{i}$ in the tables below) where $w$ is a Coxeter element in $W\left(E_{6}\right)$. From [7] we have $\left|C_{W}(w)\right|=144$. Hence the centralizer is given by

$$
C_{W}(w)=\langle w\rangle \times\left\langle-w^{6}\right\rangle \times W\left(A_{2}\right),
$$

where $A_{2}$ is orthogonal to the $E_{6}$. Since $\mathfrak{c}$ lives in the $E_{6}$ Levi subalgebra and $w^{6}$ acts by -1 on $\mathfrak{c}$, the inequalities of Lemma 7.5 become equalities for $U=\left\langle-w^{6}\right\rangle \times W\left(A_{2}\right)$. Hence $W(\mathfrak{c}, \theta) \simeq \mu_{12}$ in these eight cases.

Example 2: In type $E_{8}$ there are four cases $\left(6_{h}\right.$ through $6_{k}$ ) where $w$ is a Coxeter element in $W\left(D_{4}\right)$. Let $\Delta_{4}=\left\{\beta_{1}, \ldots, \beta_{4}\right\}$ be a base of the corresponding root subsystem of type $D_{4}$. The subgroup of $W\left(E_{8}\right)$ permuting $\Delta_{4}$ is a symmetric group $S_{3}$. We may choose the Coxeter element $w$ to be centralized by this $S_{3}$, and $\mathfrak{c}$ is a line in the span of the co-root vectors $\left\{d \check{\beta}_{i}(1)\right\}$. The roots of $E_{8}$ orthogonal to $\Delta_{4}$ form another system of type $D_{4}$, hence there is a subgroup $W_{2} \simeq W\left(D_{4}\right)$ fixing each root in $\Delta_{4}$ and therefore acting trivially on $\mathfrak{c}$. Since $S_{3}$ normalizes $\Delta_{4}$ it also normalizes $W_{2}$. From [7] we have $\left|C_{W}(w)\right|=6 \cdot 6 \cdot 192$, so the inequalities of Lemma 7.5 hold for $U \simeq S_{3} \ltimes W\left(D_{4}\right)$. Hence $W(\mathfrak{c}, \theta) \simeq \mu_{6}$ in these four cases.

Example 3: In type $E_{7}$ there are two cases $\left(9_{a}\right.$ and $\left.9_{b}\right)$ where $w$ is the square of a Coxeter element and we have $C_{W}(w)=\langle-w\rangle \simeq \mu_{18}$. Since $w$ is $\mathbb{Z}$-regular, Lemma 7.5 only gives the inequalities

$$
9 \leq|W(\mathfrak{c}, \theta)| \leq 18 .
$$

In fact, we have $W(\mathfrak{c}, \theta) \simeq \mu_{18}$ and $\mu_{9}$ in cases $9_{a}$ and $9_{b}$, respectively. This shows that, in general, $W(\mathfrak{c}, \theta)$ depends on $\theta$, and not just on $w$. We will return to this example after sharpening our lower bound, as follows.

For any subset $J \subset\{1, \ldots, \ell\}$ let $R_{J}$ be the root subsystem generated by $\left\{\alpha_{j}: j \in J\right\}$, let $W_{J}$ be Weyl group of $R_{J}$ and let $\mathfrak{g}_{J}$ be the subalgebra of $\mathfrak{g}$ generated by the root spaces $\mathfrak{g}_{\alpha}$ for $\alpha \in R_{J}$. If the action of $\theta$ on $\mathfrak{t}$ is given by an element $w \in W_{J}$ then $\theta$ induces an automorphism $\theta_{J}$ of $\mathfrak{g}_{J}$.

Lemma 7.6 Suppose $\theta$ normalizes the Cartan subalgebra $\mathfrak{t}$ and has image $w \in W_{J}$ for some subset $J \subset\{1, \ldots, \ell\}$ such that the following conditions hold.

1. $\theta$ is conjugate to an automorphism $\theta^{\prime}=\operatorname{Ad}(t)$ where $t \in T$ satisfies $\alpha_{j}(t)=\zeta$ for all $j \in J$;

2. The rank of $w$ on $\mathfrak{t}$ is equal to the rank of $\theta$; 


\section{The principal automorphisms of $\mathfrak{g}_{J}$ of order $m$ have rank equal to the rank of $\theta$. \\ 4. $w$ is $\mathbb{Z}$-regular in $W_{J}$;}

Then there is an embedding $C_{W_{J}}(w) \hookrightarrow W(\mathfrak{c}, \theta)$.

Proof: Condition 1 means there is $g \in G$ such that the automorphism

$$
\theta^{\prime}=g \theta g^{-1}=\operatorname{Ad}(t),
$$

where $t \in T$ satisfies $\alpha_{j}(t)=\zeta$ for all $j \in J$. We have $t=\check{\rho}_{J}(\zeta) z$ where $\check{\rho}_{J}$ is half the sum of the positive co-roots of $R_{J}$ (with respect to $\Delta_{J}$ ) and $z \in \operatorname{ker} \alpha_{j}$ for all $j \in J$.

Condition 2 means that the eigenspace $\mathfrak{c}:=\mathfrak{t}(w, \zeta)$ is a Cartan subspace for $\theta$. Note that $\mathfrak{c} \subset \mathfrak{g}_{J}$. Let $\mathfrak{c}_{J}$ be a Cartan subspace for the automorphism

$$
\theta_{J}^{\prime}:=\left.\theta^{\prime}\right|_{\mathfrak{g}_{J}}=\operatorname{Ad}\left(\check{\rho}_{J}(\zeta)\right) \in G_{J},
$$

where $G_{J}=\operatorname{Aut}\left(\mathfrak{g}_{J}\right)^{\circ}$.

As $\theta_{J}^{\prime}$ is principal of order $m$, we have $\operatorname{dim} \mathfrak{c}_{J}=\operatorname{dim} \mathfrak{c}$, by condition 3 .

Now $\mathfrak{c}^{\prime}:=\operatorname{Ad}(g) \mathfrak{c}$ is a Cartan subspace for $\theta^{\prime}$ in $\mathfrak{g}\left(\theta^{\prime}, \zeta\right)$, and the latter subspace contains $\mathfrak{g}_{J}\left(\theta_{J}^{\prime}, \zeta\right)$, which in turn contains $\mathfrak{c}_{J}$. Thus $\mathfrak{c}^{\prime}$ and $\mathfrak{c}_{J}$ are two Cartan subspaces in $\mathfrak{g}\left(\theta^{\prime}, \zeta\right)$, so there is $h \in G^{\theta^{\prime}}$ such that $\operatorname{Ad}(h g) \mathfrak{c}=\operatorname{Ad}(h) \mathfrak{c}^{\prime}=\mathfrak{c}_{J}[19$, Thm. 2.5]. Conjugation by $h g$ gives an isomorphism

$$
W_{G}(\mathfrak{c}, \theta) \stackrel{\sim}{\longrightarrow} W_{G}\left(\mathfrak{c}_{J}, \theta^{\prime}\right) .
$$

Since the latter group contains $W_{G_{J}}\left(\mathfrak{c}_{J}, \theta_{J}^{\prime}\right)$, we have an embedding

$$
W_{G_{J}}\left(\mathfrak{c}_{J}, \theta_{J}^{\prime}\right) \hookrightarrow W_{G}(\mathfrak{c}, \theta) .
$$

Let $\mathfrak{t}_{J}=\mathfrak{t} \cap \mathfrak{g}_{J}$ and let $\mathfrak{t}_{J}^{\prime}$ be a $\theta_{J}^{\prime}$-stable Cartan subalgebra of $\mathfrak{g}_{J}$ containing $\mathfrak{c}_{J}$. Then there is $b \in G_{J}$ such that $\operatorname{Ad}(b) \mathfrak{t}_{J}^{\prime} \subset \mathfrak{t}_{J}$, so $b \theta_{J}^{\prime} b^{-1}$ normalizes $\mathfrak{t}_{J}$ and $\mathfrak{c}_{J}^{\prime}:=\operatorname{Ad}(b) \mathfrak{c}_{J}$ is a Cartan subspace for $b \theta_{J}^{\prime} b^{-1}$ contained in $\mathfrak{t}_{J}$. Let $w^{\prime} \in W_{J}$ be the element induced by $b \theta_{J}^{\prime} b^{-1}$. We now have two elements $w, w^{\prime} \in$ $W_{J}$ having equidimensional $\zeta$-eigenspaces $\mathfrak{c}$ and $\mathfrak{c}_{J}^{\prime}$ in $\mathfrak{t}_{J}$.

The one-parameter subgroups of $G_{J}$ which centralize $\mathfrak{t}_{J}$ form a lattice giving a $\mathbb{Z}$-form $\check{X}_{J}$ of $\mathfrak{t}_{J}$. Let $A$ be the cyclotomic subring of $\mathbb{C}$ generated by $z=e^{2 p i i / m}$ and let $\pi: A \rightarrow k$ be the ring homomorphism sending $z \mapsto \zeta$. Since the map $\pi: \mu_{m}\left(\mathbb{C}^{\times}\right) \rightarrow \mu_{m}\left(k^{\times}\right)$is an isomorphism, it follows that the $z$ eigenspaces of $w$ and $w^{\prime}$ in $\check{X}_{J} \otimes \mathbb{C}$ have the same dimension.

Now $w$ is $k$-regular on $\mathfrak{t}_{J}=k \otimes \check{X}_{J}$, by condition 4. Hence $w$ is $\mathbb{C}$-regular on $\mathbb{C} \otimes \check{X}_{J}$, by Prop. 3.2. By $[29,6.4]$, the elements $w$ and $w^{\prime}$ are conjugate in $W_{J}$, so $w^{\prime}$ is $k$-regular on $\mathfrak{t}_{J}$. Hence the principal automorphism $b \theta_{J}^{\prime} b^{-1}$ of $\mathfrak{g}_{J}$ has regular vectors in $\operatorname{Ad}(b) \mathfrak{c}_{J}$, so the principal automorphism $\theta_{J}^{\prime}$ has regular vectors in $\mathfrak{c}_{J}$. It now follows from Cor. 7.3 that $W_{G_{J}}\left(\mathfrak{c}_{J}, \theta_{J}^{\prime}\right) \simeq C_{W_{J}}\left(w^{\prime}\right) \simeq C_{W_{J}}(w)$.

Remarks: 1 . In practice, condition 1 means the normalized Kac diagram of $\theta$ can be conjugated under the affine Weyl group $W_{\text {aff }}(R)$ to a (usually un-normalized) Kac diagram with 1 on each node for $j \in J$. We will see that condition 1 is verified as a byproduct of the normalization algorithm. 
2. The element $w$ is usually elliptic in $W_{J}$. When this holds, condition 3 is implied by conditions 2 and 4, as follows from Prop. 5.5.

3. Recall that the order of $C_{W_{J}}(w)$ is the product of those degrees of $W_{J}$ which are divisible by the order $m$ of $w$. Thus the lower bound in Prop. 7.6 is completely explicit.

Example 3 revisited: Recall that $G$ has type $E_{7}$ and $w$ is the square of a Coxeter element. We give the normalized Kac diagram for each $\theta$, the un-normalized diagram for each $\theta^{\prime}$, whose subdiagram of 1 's determines $J$.

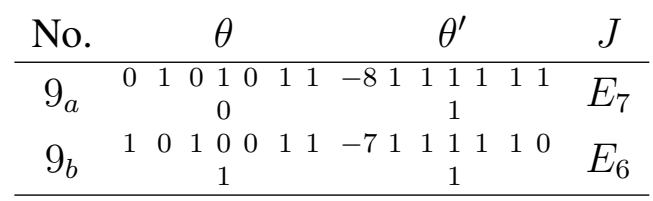

Lemma 7.6 shows that $9_{a}$ has little Weyl group $W(\mathfrak{c}, \theta) \simeq \mu_{18}$, but does not decide case $9_{b}$, which we treat using invariant theory (see section 10).

\subsection{Stable isotropy groups}

Assume that $\theta \in \operatorname{Aut}(\mathfrak{g})$ gives a stable grading $\mathfrak{g}=\oplus_{i \in \mathbb{Z} / m} \mathfrak{g}_{i}$. By definition there is a regular semisimple element $v \in \mathfrak{g}_{1}$ whose isotropy subgroup in $G_{0}$ is finite. Fix a Cartan subspace $\mathfrak{c} \subset \mathfrak{g}_{1}$ and let $S$ be the unique maximal torus in $G$ centralizing c. In the proof of Lemma 5.6 we saw that $C_{G}(v)$ is a torus, so we must have $C_{G}(v)=S$. It follows that all stable vectors in $\mathfrak{c}$ have the same isotropy group in $G_{0}$, equal to

$$
S_{0}:=S \cap G_{0}
$$

We now give a more explicit description of $S_{0}$.

First, $S_{0}$ is contained in the fixed-point subgroup $S^{\theta}$, which is finite of order

$$
\left|S^{\theta}\right|=\operatorname{det}\left(1-\left.\theta\right|_{X^{*}(S)}\right)
$$

Let $N(S)$ be the normalizer of $S$ in $G$. Then $N(S)^{\theta}$ meets all components of $G^{\theta}$, and it follows from Cor. 7.4 that the inclusion $S^{\theta} \hookrightarrow G^{\theta}$ induces an isomorphism

$$
S^{\theta} / S_{0} \simeq G^{\theta} / G_{0}
$$

This quotient depends only on the image $\vartheta$ of $\theta$ in the component group of $\operatorname{Aut}(\mathfrak{g})$. To see this, let

$$
G_{s c} \stackrel{\pi}{\longrightarrow} G
$$

be the simply-connected covering of $G$ and set $Z=\operatorname{ker} \pi$. Then $\theta$ and $\vartheta$ lift to automorphisms of $G_{s c}$ which we again denote by $\theta$ and $\vartheta$. Since $G_{s c}^{\theta}$ is connected and $\theta=\vartheta$ on $Z$, we have an exact sequence

$$
1 \longrightarrow Z^{\vartheta} \longrightarrow G_{s c}^{\theta} \longrightarrow G_{0} \longrightarrow 1
$$

which restricts to an exact sequence

$$
1 \longrightarrow Z^{\vartheta} \longrightarrow S_{s c}^{\theta} \longrightarrow S_{0} \longrightarrow 1
$$


where $S_{s c}=\pi^{-1}(S)$. Since

$$
\left|S^{\theta}\right|=\left|S_{s c}^{\theta}\right|
$$

it follows that we have another exact sequence

$$
1 \longrightarrow S_{0} \longrightarrow S^{\theta} \longrightarrow Z /(1-\vartheta) Z \longrightarrow 1 \text {. }
$$

On the other hand, $Z /(1-\vartheta) Z$ is isomorphic to the subgroup $\Omega_{\vartheta} \subset \widetilde{W}_{\text {aff }}(R, \vartheta)$ stabilizing the alcove $C$. The group $\Omega_{\vartheta}$ acts as symmetries of the twisted affine Dynkin diagram $D\left({ }^{e} R\right)$. These groups are well-known if $e=1$; for $e>1, \Omega_{\vartheta}$ is the full symmetry group of $D\left({ }^{e} R\right)$ and has order 1 or 2 . It follows that if $\theta$ is stable then the isotropy group $S_{0}$ fits into an exact sequence

$$
1 \longrightarrow S_{0} \longrightarrow S^{\theta} \longrightarrow \Omega_{\vartheta} \longrightarrow 1
$$

The groups $S_{0}$ are tabulated for exceptional groups in Sect. 8.1.

\subsection{Stable orbits and elliptic curves}

Certain remarkable stable gradings have appeared in recent work of Barghava and Shankar on the

\begin{tabular}{|c|c|c|c|c|c|}
\hline$m$ & Kac coord. & $W(\mathfrak{c}, \theta)$ & degrees & $G_{0}$ & $\mathfrak{g}_{1}$ \\
\hline 2 & $1 \Longrightarrow 0$ & $\mathrm{SL}_{2}(\mathbb{Z} / 2)$ & 2,3 & $\mathrm{SL}_{2} / \boldsymbol{\mu}_{2}$ & $\operatorname{Sym}^{4}(\mathbf{2})$ \\
\hline 3 & $00 \Leftarrow 1$ & $\mathrm{SL}_{2}(\mathbb{Z} / 3)$ & 4,6 & $\mathrm{SL}_{3} / \boldsymbol{\mu}_{3}$ & $\operatorname{Sym}^{3}(\mathbf{3})$ \\
\hline 4 & $000 \Leftarrow 10$ & $\boldsymbol{\mu}_{2} \times \mathrm{SL}_{2}(\mathbb{Z} / 4)$ & 8,12 & $\left(\mathrm{SL}_{2} \times \mathrm{SL}_{4}\right) / \boldsymbol{\mu}_{4}$ & $2 \bowtie \operatorname{Sym}^{2}(4)$ \\
\hline 5 & $\begin{array}{cccccccc}0 & 0 & 0 & 1 & 0 & 0 & 0 & 0 \\
& 0 & & & & & \end{array}$ & $\boldsymbol{\mu}_{5} \times \mathrm{SL}_{2}(\mathbb{Z} / 5)$ & 20,30 & $\left(\mathrm{SL}_{5} \times \mathrm{SL}_{5}\right) / \boldsymbol{\mu}_{5}$ & $5 \otimes \Lambda^{2} 5$ \\
\hline
\end{tabular}
average rank of elliptic curves ([1], [2]). These gradings have periods $m=2,3,4,5$ and are of types ${ }^{2} A_{2},{ }^{3} D_{4},{ }^{2} E_{6}, E_{8}$ respectively, as tabulated below. Here $\mathbf{d}$ stands for the natural representation of $\mathrm{SL}_{d}$.

For each $m=2,3,4,5$ the isotropy subgroup $S_{0}$ is isomorphic to $\boldsymbol{\mu}_{m} \times \boldsymbol{\mu}_{m}$ and the little Weyl group $W(\mathfrak{c}, \theta)$ is isomorphic to the group $W_{m}$ with presentation

$$
W_{m}=\left\langle s, t: s^{m}=t^{m}=1, \quad s t s=t s t\right\rangle .
$$

(Note that $W_{m}$ is infinite for $m>5$.) The exact sequence

$$
1 \longrightarrow S_{0} \longrightarrow N_{G_{0}}(\mathfrak{c}) \longrightarrow W(\mathfrak{c}, \theta) \longrightarrow 1
$$


gives a homomorphism $W(\mathfrak{c}, \theta) \rightarrow \operatorname{Aut}\left(S_{0}\right)=\mathrm{GL}_{2}(\mathbb{Z} / m \mathbb{Z})$ with image $\mathrm{SL}_{2}(\mathbb{Z} / m \mathbb{Z})$ and split kernel $\left\langle\theta^{e}\right\rangle \simeq \boldsymbol{\mu}_{m / e}$, as tabulated above (see also [25]).

In each case the number $|R|$ of roots is equal to $m \cdot(m-1) \cdot(12 / b)$, where $b=4,3,2,1$ is the maximal number of bonds between two nodes in the twisted affine diagram $D\left({ }^{e} R\right)$. We have $\operatorname{dim} G_{0}=|R| / m$ and the degrees $d_{1}<d_{2}$ have the property that $3 d_{1}=2 d_{2}=|R| /(m-1)$. Let $I, J \in k[\mathfrak{c}]^{W(\mathfrak{c}, \theta)}$ be homogeneous generators of degrees $d_{1}, d_{2}$. The discriminant on $\mathfrak{t}$ (product of all the roots in $R$ ) has restriction to $\mathfrak{c}$ given by $D^{m-1}$ (up to nonzero scalar), where $D=-4 I^{3}-27 J^{2}$. The stable vectors $v \in \mathfrak{c}$ are those where $D(v) \neq 0$, and each stable vector $v$ corresponds to an elliptic curve $E_{v}$ with equation

$$
y^{2}=x^{3}+I(v) \cdot x+J(v)
$$

whose $m$-torsion group $E_{v}[m]$ is isomorphic (as an algebraic group over $k$ ) to $S_{0}$. For more information, along with some generalizations to hyperelliptic curves, see [10].

\section{Classification of stable gradings}

Let $\theta \in G \vartheta$ be an automorphism of $\mathfrak{g}$ whose order $m$ is invertible in $k$, associated to the grading $\mathfrak{g}=\oplus_{i \in \mathbb{Z} / m} \mathfrak{g}_{i}$. After conjugating $\theta$ by an element of $G$ we may assume that $\mathfrak{t}$ is the canonical Cartan subalgebra of $\theta$. Then $\left.\theta\right|_{\mathfrak{t}}=w \vartheta$, for some $w \in W$. In section 5 we have seen that $\theta$ is stable if and only if $w \vartheta$ is an elliptic $\mathbb{Z}$-regular automorphism of $R$, in which case $\theta$ is $G$-conjugate to $\check{\rho}(\zeta) \vartheta$ for some/any root of unity $\zeta \in k^{\times}$of order $m$. Moreover, the $G$-conjugacy class of $\theta$ is completely determined by its order $m$. The values of $m$ which can arise are the orders of elliptic $\mathbb{Z}$-regular automorphisms of $R$ in $W \vartheta$; these are classified in [29].

For example, the elliptic $\mathbb{Z}$-regular elements in $W \vartheta$ of maximal order are the $\vartheta$-Coxeter elements, whose order is the $\vartheta$-Coxeter number

$$
h_{\vartheta}=e \cdot\left(b_{1}+b_{2}+\cdots+b_{\ell_{\vartheta}}\right)
$$

(see (2)). These form a single $W$-conjugacy class in $W \vartheta$, representatives of which include elements of the form $w \vartheta$, where $w$ is the product, in any order, of one reflection $r_{i}$ taken from each of the $\vartheta$-orbits on simple reflections.

For any algebraically closed field $k$ in which $h_{\vartheta}$ is invertible and any $\zeta \in k^{\times}$of order $h_{\vartheta}$, the automorphism

$$
\theta_{\text {cox }}=\operatorname{Ad}(\check{\rho}(\zeta)) \vartheta \in \operatorname{Aut}(\mathfrak{g})
$$

is stable of order $h_{\vartheta}$ and acts on its canonical Cartan subalgebra via a $\vartheta$-Coxeter element. The Kac coordinates of $\theta_{\text {cox }}$ have $s_{i}=1$ for all $i \in\left\{0, \ldots, \ell_{\vartheta}\right\}$ and are already normalized.

For $m<h_{\vartheta}$ the automorphism $\check{\rho}(\zeta) \vartheta$ corresponds to a point in $\mathcal{A}_{\mathbb{Q}}^{\vartheta}$ with un-normalized coordinates $s_{i}=1$ for $i \neq 0$ and $s_{0}=1+\left(m-h_{\vartheta}\right) / e$ (see 2.4). Here we must apply the normalization algorithm to obtain normalized Kac coordinates. By (13) these normalized Kac diagrams will be invariant under the symmetry group of the diagram $D\left({ }^{e} R\right)$. The resulting classification of the stable gradings in all types is tabulated for exceptional Lie algebras in section 8.1 and for classical Lie algebras in section 8.2 . 


\subsection{Stable gradings of exceptional Lie algebras}

Here we tabulate the stable gradings for exceptional Lie algebras, along with the corresponding elliptic $\mathbb{Z}$-regular element $w \vartheta \in W \vartheta$ and the isotropy group $S_{0}$ (see section 7.3). The column labelled $A$ will be explained in section 8.3.

Table 2: The stable gradings for $E_{6}$

\begin{tabular}{|c|c|c|c|c|c|}
\hline$m$ & un-normaliz & normalized & $w$ & $S_{0}$ & $A$ \\
\hline $12=h_{\vartheta}$ & 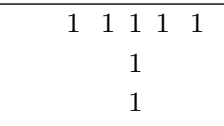 & $\begin{array}{ccccc}1 & 1 & 1 & 1 & 1 \\
& & 1 & & \\
& & & & \\
\end{array}$ & $E_{6}$ & 1 & $E_{6}$ \\
\hline 9 & 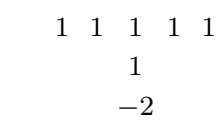 & $\begin{array}{ccccc}1 & 1 & 0 & 1 & 1 \\
& & 1 & & \\
& & & & \end{array}$ & $E_{6}\left(a_{1}\right)$ & 1 & $E_{6}\left(a_{1}\right)$ \\
\hline 6 & 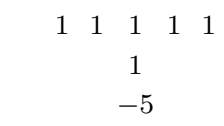 & $\begin{array}{ccccc}1 & 0 & 1 & 0 & 1 \\
& & 0 & & \\
& & 1 & & \end{array}$ & $E_{6}\left(a_{2}\right)$ & 1 & $E_{6}\left(a_{3}\right)$ \\
\hline 3 & 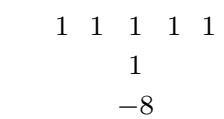 & $\begin{array}{ccccc}0 & 0 & 1 & 0 & 0 \\
& & 0 & & \end{array}$ & $3 A_{2}$ & $\boldsymbol{\mu}_{3} \times \boldsymbol{\mu}_{3}$ & - \\
\hline
\end{tabular}

Table 3: The stable gradings for ${ }^{2} E_{6}$

\begin{tabular}{ccccc}
\hline$m$ & un-normalized & normalized & $w \vartheta$ & $S_{0}$ \\
\hline $18=h_{\vartheta}$ & $111 \Leftarrow 11$ & $111 \Leftarrow 11$ & $-E_{6}\left(a_{1}\right)$ & 1 \\
12 & $-211 \Leftarrow 11$ & $110 \Leftarrow 11$ & $-E_{6}$ & 1 \\
6 & $-511 \Leftarrow 11$ & $100 \Leftarrow 10$ & $-\left(3 A_{2}\right)$ & 1 \\
4 & $-611 \Leftarrow 11$ & $000 \Leftarrow 10$ & $-D_{4}\left(a_{1}\right)$ & $\boldsymbol{\mu}_{4} \times \boldsymbol{\mu}_{4}$ \\
2 & $-711 \Leftarrow 11$ & $000 \Leftarrow 01$ & -1 & $\boldsymbol{\mu}_{2}^{6}$ \\
\hline
\end{tabular}

Table 4: The stable gradings for $E_{7}$

\begin{tabular}{|c|c|c|c|c|c|}
\hline$m$ & un-normalized & normalized & $w$ & $S_{0}$ & $A$ \\
\hline $18=h_{\vartheta}$ & $\begin{array}{lllllll}1 & 1 & 1 & 1 & 1 & 1 & 1 \\
& & & & 1 & & \\
\end{array}$ & 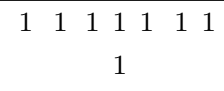 & $E_{7}$ & 1 & $E_{7}$ \\
\hline 14 & 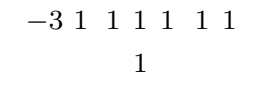 & $\begin{array}{ccccccc}1 & 1 & 1 & 0 & 1 & 1 & 1 \\
& & & & 1 & & \\
\end{array}$ & $E_{7}\left(a_{1}\right)$ & 1 & $E_{7}\left(a_{1}\right)$ \\
\hline 6 & 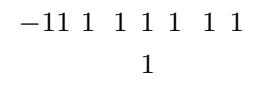 & $\begin{array}{ccccccc}1 & 0 & 0 & 1 & 0 & 0 & 1 \\
& & & & 0 & & \\
\end{array}$ & $E_{7}\left(a_{4}\right)$ & 1 & $E_{7}\left(a_{5}\right)$ \\
\hline 2 & $\begin{array}{cccccc}-1511 & 1 & 1 & 1 & 1 & 1 \\
& & & 1 & & \end{array}$ & $\begin{array}{ccccccc}0 & 0 & 0 & 0 & 0 & 0 & 0 \\
& & & & 1 & & \\
\end{array}$ & $7 A_{1}$ & $\boldsymbol{\mu}_{2}^{6}$ & - \\
\hline
\end{tabular}


Table 5: The stable gradings for $E_{8}$

\begin{tabular}{|c|c|c|c|c|c|}
\hline$m$ & un-normalized & normalized & $w$ & $S_{0}$ & $A$ \\
\hline $30=h_{\vartheta}$ & $\begin{array}{cccccccc}1 & 1 & 1 & 1 & 1 & 1 & 1 & 1 \\
& & 1 & & & & & \end{array}$ & $\begin{array}{llllllll}1 & 1 & 1 & 1 & 1 & 1 & 1 & 1 \\
& & 1 & & & & & \end{array}$ & $E_{8}$ & 1 & $E_{8}$ \\
\hline 24 & $\begin{array}{cccccc}11 & 1 & 1 & 1 & 1 & 1-5 \\
& & 1 & & & \end{array}$ & 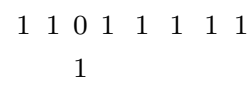 & $E_{8}\left(a_{1}\right)$ & 1 & $E_{8}\left(a_{1}\right)$ \\
\hline 20 & $\begin{array}{ccccccc}1 & 1 & 1 & 1 & 1 & 1 & 1-9 \\
& & & & & & \end{array}$ & $\begin{array}{cccccccc}1 & 1 & 0 & 1 & 0 & 1 & 1 & 1 \\
& & 1 & & & & & \end{array}$ & $E_{8}\left(a_{2}\right)$ & 1 & $E_{8}\left(a_{2}\right)$ \\
\hline 15 & $\begin{array}{ccccccc}1 & 1 & 1 & 1 & 1 & 1 & 1-14 \\
& & & & & & \end{array}$ & $\begin{array}{cccccccc}1 & 0 & 1 & 0 & 1 & 0 & 1 & 1 \\
& & 0 & & & & & \end{array}$ & $E_{8}\left(a_{5}\right)$ & 1 & $E_{8}\left(a_{4}\right)$ \\
\hline 12 & 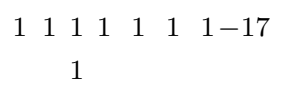 & 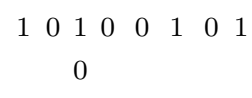 & $E_{8}\left(a_{3}\right)$ & 1 & $E_{8}\left(a_{5}\right)$ \\
\hline 10 & 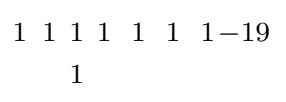 & 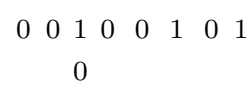 & $E_{8}\left(a_{6}\right)=-2 A_{4}$ & 1 & $E_{8}\left(a_{6}\right)$ \\
\hline 8 & $\begin{array}{ccccccc}1 & 1 & 1 & 1 & 1 & 1 & 1-21 \\
& & & & & & \\
& & \end{array}$ & $\begin{array}{cccccccc}0 & 0 & 1 & 0 & 0 & 0 & 1 & 0 \\
& & 0 & & & & & \end{array}$ & $D_{8}\left(a_{3}\right)$ & $\boldsymbol{\mu}_{2} \times \boldsymbol{\mu}_{2}$ & - \\
\hline 6 & $\begin{array}{ccccccc}1 & 1 & 1 & 1 & 1 & 1 & 1-23 \\
& & 1 & & & & \end{array}$ & $\begin{array}{cccccccc}0 & 0 & 0 & 1 & 0 & 0 & 0 & 1 \\
& & 0 & & & & & \end{array}$ & $E_{8}\left(a_{8}\right)=-4 A_{2}$ & 1 & $E_{8}\left(a_{7}\right)$ \\
\hline 5 & $\begin{array}{llllllll}1 & 1 & 1 & 1 & 1 & 1 & 1-24 \\
& & 1 & & & & & \end{array}$ & $\begin{array}{cccccccc}0 & 0 & 0 & 1 & 0 & 0 & 0 & 0 \\
& & 0 & & & & & \\
\end{array}$ & $2 A_{4}$ & $\boldsymbol{\mu}_{5} \times \boldsymbol{\mu}_{5}$ & - \\
\hline 4 & $\begin{array}{ccccccc}1 & 1 & 1 & 1 & 1 & 1 & 1-25 \\
& & & & & & \end{array}$ & $\begin{array}{cccccccc}0 & 0 & 0 & 0 & 1 & 0 & 0 & 0 \\
& 0 & & & & & & \end{array}$ & $2 D_{4}\left(a_{1}\right)$ & $\boldsymbol{\mu}_{2}^{4}$ & - \\
\hline 3 & 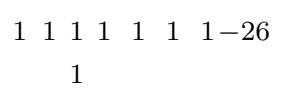 & 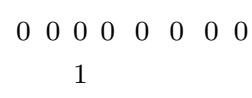 & $4 A_{2}$ & $\boldsymbol{\mu}_{3}^{4}$ & - \\
\hline 2 & 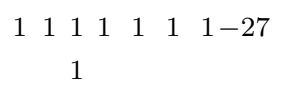 & 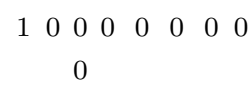 & $8 A_{1}=-1$ & $\boldsymbol{\mu}_{2}^{8}$ & - \\
\hline
\end{tabular}

Table 6: The stable gradings for $F_{4}$

\begin{tabular}{cccccc}
\hline$m$ & un-normalized & normalized & $w$ & $S_{0}$ & $A$ \\
\hline $12=h_{\vartheta}$ & $111 \Rightarrow 11$ & $111 \Rightarrow 11$ & $F_{4}$ & 1 & $F_{4}$ \\
8 & $-311 \Rightarrow 11$ & $111 \Rightarrow 01$ & $B_{4}$ & $\boldsymbol{\mu}_{2}$ & $F_{4}\left(a_{1}\right)$ \\
6 & $-511 \Rightarrow 11$ & $101 \Rightarrow 01$ & $F_{4}\left(a_{1}\right)$ & 1 & $F_{4}\left(a_{2}\right)$ \\
4 & $-711 \Rightarrow 11$ & $101 \Rightarrow 00$ & $D_{4}\left(a_{1}\right)$ & $\boldsymbol{\mu}_{2} \times \boldsymbol{\mu}_{2}$ & $F_{4}\left(a_{3}\right)$ \\
3 & $-811 \Rightarrow 11$ & $001 \Rightarrow 00$ & $A_{2}+\tilde{A}_{2}$ & $\boldsymbol{\mu}_{3} \times \boldsymbol{\mu}_{3}$ & - \\
2 & $-911 \Rightarrow 11$ & $010 \Rightarrow 00$ & $4 A_{1}$ & $\boldsymbol{\mu}_{2}^{4}$ & - \\
\hline
\end{tabular}

Table 7: The stable gradings for $G_{2}$

\begin{tabular}{cccccc}
\hline$m$ & un-normalized & normalized & $w$ & $S_{0}$ & $A$ \\
\hline $6=h_{\vartheta}$ & $11 \Rightarrow 1$ & $11 \Rightarrow 1$ & $G_{2}$ & 1 & $G_{2}$ \\
3 & $-21 \Rightarrow 1$ & $11 \Rightarrow 0$ & $A_{2}$ & $\boldsymbol{\mu}_{3}$ & $G_{2}\left(a_{1}\right)$ \\
2 & $-31 \Rightarrow 1$ & $01 \Rightarrow 0$ & $A_{1}+\tilde{A}_{1}$ & $\boldsymbol{\mu}_{2} \times \boldsymbol{\mu}_{2}$ & - \\
\hline
\end{tabular}


Table 8: The stable gradings for ${ }^{3} D_{4}$

\begin{tabular}{|c|c|c|c|c|}
\hline$m$ & un-normalized & normalized & $w \vartheta \in W\left(F_{4}\right)$ & $S_{0}$ \\
\hline $12=h_{\vartheta}$ & $11 \Leftarrow 1$ & $11 \Leftarrow 1$ & $F_{4}$ & 1 \\
\hline 6 & $-11 \Leftarrow 1$ & $10 \Leftarrow 1$ & $F_{4}\left(a_{1}\right)$ & 1 \\
\hline 3 & $-21 \Leftarrow 1$ & $00 \Leftarrow 1$ & $A_{2}+\tilde{A}_{2}$ & $\boldsymbol{\mu}_{3} \times \boldsymbol{\mu}_{3}$ \\
\hline
\end{tabular}

\subsection{Stable gradings of classical Lie algebras}

Here we tabulate the stable gradings of classical Lie algebras. For inner type $A_{n}$ the only stable grading is the Coxeter one, so we omit this case.

\subsubsection{Type ${ }^{2} A_{\ell}$}

The stable gradings in type ${ }^{2} A_{\ell}$ correspond to divisors of $\ell$ and $\ell+1$, each having odd quotient $d=m / 2$. Conjugacy classes in the symmetric group are denoted by their partitions. For example, $\left[d^{2 k+1}\right]$ consists of the products of $2 k+1$ disjoint $d$-cycles.

Table 9: The stable gradings for ${ }^{2} A_{2}$

\begin{tabular}{cccc}
\hline$m=2 d$ & Kac diagram & $w \vartheta$ & $S_{0}$ \\
\hline $6=h_{\vartheta}$ & $1 \equiv 1$ & $-1 \times[3]$ & 1 \\
2 & $1 \equiv 0$ & $-\left[1^{3}\right]$ & $\boldsymbol{\mu}_{2} \times \boldsymbol{\mu}_{2}$ \\
\hline
\end{tabular}

Table 10: The stable gradings for ${ }^{2} A_{2 n}, n \geq 2$

\begin{tabular}{|c|c|c|c|}
\hline$m=2 d$ & Kac diagram & $w \vartheta$ & $S_{0}$ \\
\hline $2(2 n+1)=h_{\vartheta}$ & $1 \Rightarrow 11 \cdots 1 \quad 1 \Rightarrow 1$ & $-1 \times[2 n+1]$ & 1 \\
\hline 2 & $1 \Rightarrow 0 \quad 0 \quad 0 \cdots 0 \quad 0 \Rightarrow 0$ & $-1 \times\left[1^{2 n+1}\right]$ & $\boldsymbol{\mu}_{2}^{2 n}$ \\
\hline$\frac{2(2 n+1)}{2 k+1}, \quad k>0$ & $1 \Rightarrow \underbrace{0 \cdots 0}_{A_{2 k}} 1 \underbrace{0 \cdots 0}_{A_{2 k}} 1 \cdots \underbrace{0 \cdots 0}_{A_{2 k}} \Rightarrow 1$ & $-1 \times\left[d^{2 k+1}\right]$ & $\boldsymbol{\mu}_{2}^{2 k}$ \\
\hline$\frac{2 n}{k}, \quad 1<\frac{n}{k}$ odd & $1 \Rightarrow \underbrace{0 \cdots 0}_{A_{2 k-1}} 1 \underbrace{0 \cdots 0}_{A_{2 k-1}} 1 \cdots 1 \underbrace{0 \cdots 0 \Rightarrow 0}_{B_{k}}$ & $-1 \times\left[d^{2 k}, 1\right]$ & $\boldsymbol{\mu}_{2}^{2 k}$ \\
\hline
\end{tabular}


Table 11: The stable gradings for ${ }^{2} A_{2 n-1}, n \geq 3$

\begin{tabular}{|c|c|c|c|c|c|c|}
\hline$m$ & & & & Kac diagram & $w \vartheta$ & $S_{0}$ \\
\hline $2(2 n-1)=h_{\vartheta}$ & & $\begin{array}{rr} & 1 \\
1 & 1\end{array}$ & & $1 \quad 1 \cdots 1 \quad 1 \Leftarrow 1$ & $-1 \times[2 n-1]$ & 1 \\
\hline $2 n \quad(n$ odd $)$ & & $\begin{array}{ll} & 1 \\
1 & 0\end{array}$ & 1 & $0 \quad 1 \cdots 1 \quad 0 \Leftarrow 1$ & $-1 \times\left[n^{2}\right]$ & 1 \\
\hline$\frac{2(2 n-1)}{2 k+1}, \quad k>0$ & $\underbrace{0}$ & $\underbrace{0}_{D_{k+1}}$ & $1 \underbrace{(}$ & $\underbrace{0 \cdots 0}_{A_{2 k}} 1 \cdots 1 \underbrace{0 \cdots 0}_{A_{2 k}} \Leftarrow 1$ & $-1 \times\left[d^{2 k+1}, 1\right]$ & $\boldsymbol{\mu}_{2}^{2 k}$ \\
\hline$\frac{2 n}{k}, \quad 1<\frac{n}{k}$ odd & $\underbrace{0}$ & $\underbrace{0}_{D_{k}}$ & $1 \underbrace{0}$ & $\underbrace{0 \cdots 0}_{A_{2 k-1}} 1 \cdots 1 \underbrace{0 \cdots 0}_{A_{2 k-1}} \Leftarrow 1$ & $-1 \times\left[d^{2 k}\right]$ & $\boldsymbol{\mu}_{2}^{2 k-2}$ \\
\hline
\end{tabular}




\subsubsection{Types $B_{n}, C_{n}$}

The stable gradings for type $B_{n}$ and $C_{n}$ correspond to divisors $k$ of $n$, with period $m=2 n / k$. The corresponding class in $W\left(B_{n}\right)=W\left(C_{n}\right)$, denoted $k B_{n / k}$, consists of the $k^{t h}$ powers of a Coxeter element.

Table 12: The stable gradings for type $B_{n}$

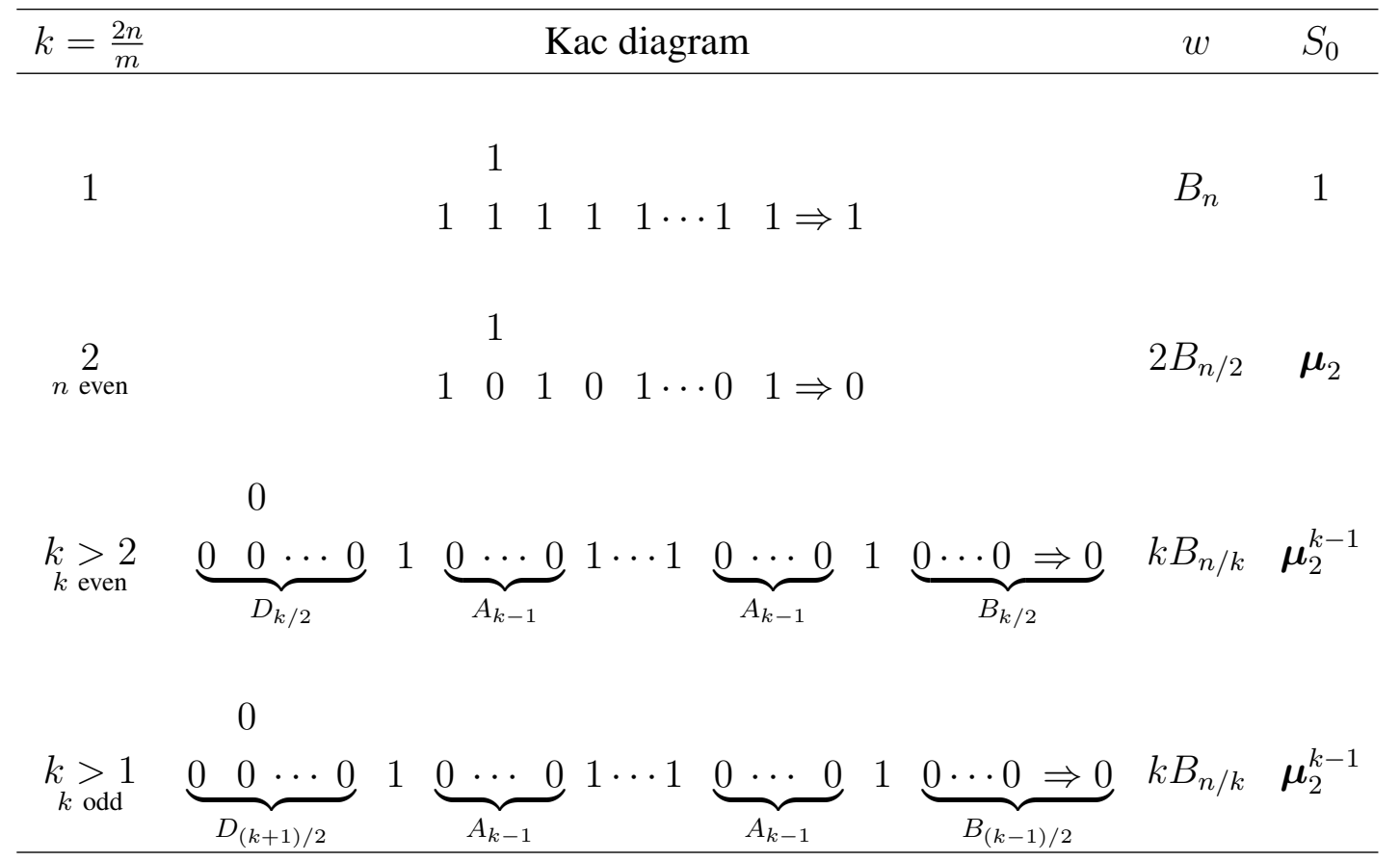

Table 13: The stable gradings for type $C_{n}$

\begin{tabular}{|c|c|c|c|}
\hline$k=\frac{2 n}{m}$ & Kac diagram & $w$ & $S_{0}$ \\
\hline 1 & $1 \Rightarrow 1 \quad 1 \cdots 1 \quad 1 \Leftarrow 1$ & $B_{n}$ & 1 \\
\hline$k>1$ & $1 \Rightarrow \underbrace{0 \cdots 0}_{A_{k-1}} 1 \underbrace{0 \cdots 0}_{A_{k-1}} 1 \cdots 1 \underbrace{0 \cdots 0}_{A_{k-1}} \Leftarrow 1$ & $k B_{n / k}$ & $\boldsymbol{\mu}_{2}^{k-1}$ \\
\hline
\end{tabular}




\subsubsection{Types $D_{n}$ and ${ }^{2} D_{n} \quad(n \geq 4)$}

The stable gradings for type $D_{n}$ correspond to even divisors $k$ of $n$ and odd divisors $\ell$ of $n-1$. The stable gradings for type ${ }^{2} D_{n}$ correspond to odd divisors $\ell$ of $n$ and even divisors $k$ of $n-1$.

Table 14: The stable gradings for type $D_{n}, n \geq 4$

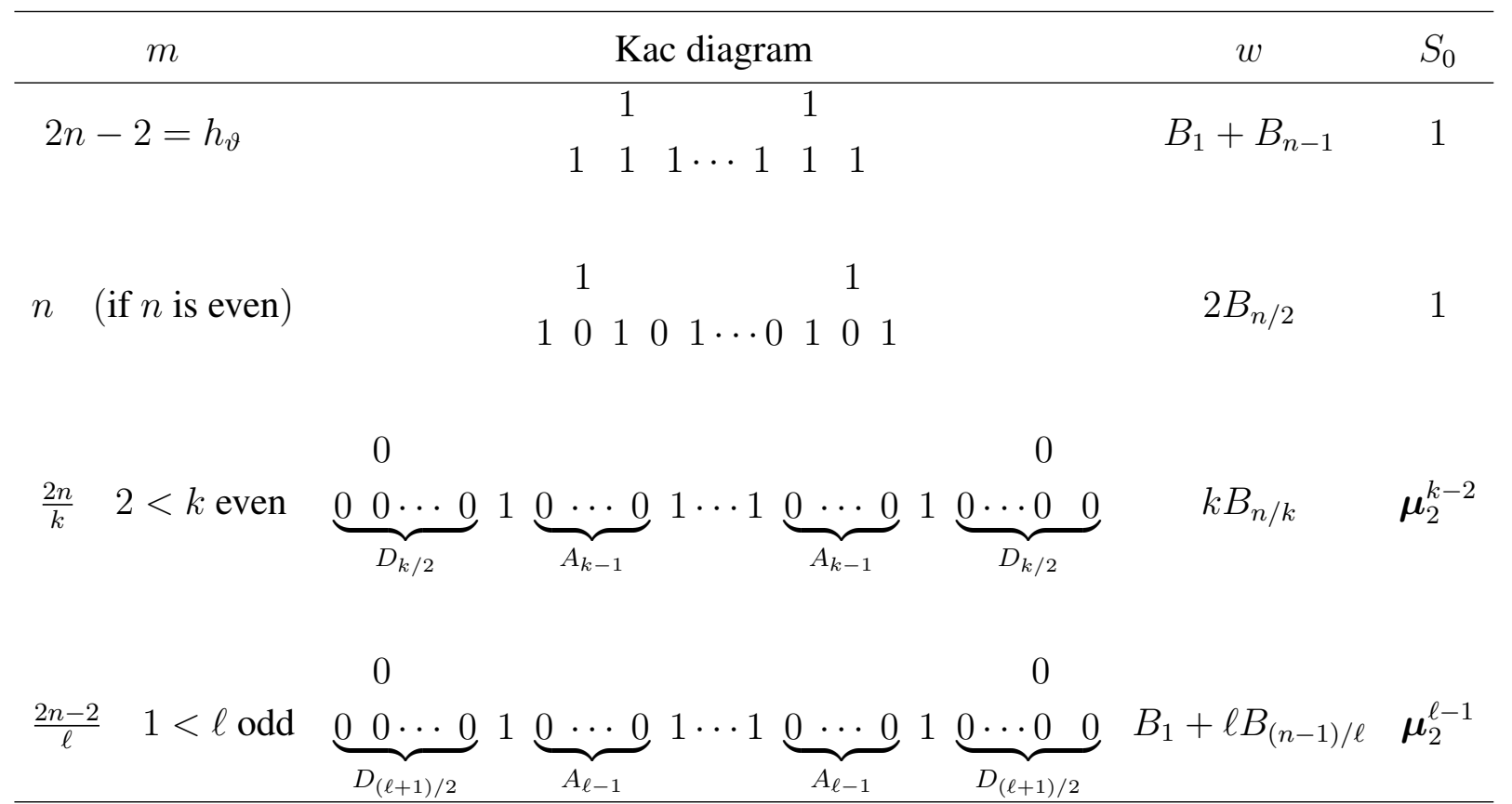

Table 15: The stable gradings for type ${ }^{2} D_{n}, n \geq 3$

\begin{tabular}{|c|c|c|c|c|}
\hline & $m$ & Kac diagram & $w$ & $S_{0}$ \\
\hline & $2 n=h_{\vartheta}$ & $1 \Leftarrow 1 \quad 1 \cdots 1 \quad 1 \Rightarrow 1$ & $B_{n}$ & 1 \\
\hline$n-1$ & (if $n$ is odd) & $0 \Leftarrow 1 \quad 0 \quad 1 \quad 0 \cdots 1 \quad 0 \quad 1 \Rightarrow 0$ & $B_{1}+2 B_{n / 2}$ & $\mu_{2} \times \mu_{2}$ \\
\hline$\frac{2 n}{\ell}$ & $2<\ell$ odd & $\underbrace{0 \Leftarrow 0 \cdots 0}_{B_{(\ell-1) / 2}} 1 \underbrace{0 \cdots 0}_{A_{\ell-1}} 1 \cdots 1 \underbrace{0 \cdots 0}_{A_{\ell-1}} 1 \underbrace{0 \cdots 0 \Rightarrow 0}_{B_{(\ell-1) / 2}}$ & $\ell B_{n / \ell}$ & $\boldsymbol{\mu}_{2}^{\ell-1}$ \\
\hline$\frac{2 n-2}{k}$ & $1<k$ even & $\underbrace{0 \Leftarrow 0 \cdots 0}_{B_{k / 2}} 1 \underbrace{0 \cdots 0}_{A_{k-1}} 1 \cdots 1 \underbrace{0 \cdots 0}_{A_{k-1}} 1 \underbrace{0 \cdots 0 \Rightarrow 0}_{B_{k / 2}}$ & $B_{1}+k B_{(n-1) / k}$ & $\boldsymbol{\mu}_{2}^{k}$ \\
\hline
\end{tabular}




\subsection{Distinguished nilpotent elements and stable gradings}

Kac coordinates of stable gradings are of two kinds, according as $s_{0}=0$ or $s_{0}=1$. Expanding on section 9 of [29], we show here that all stable gradings with $s_{0}=1$ in exceptional Lie algebras are related to distinguished nilpotent elements. For simplicity, we assume in this section only that $k$ has characteristic zero.

Let $A$ be a distinguished nilpotent element in $\mathfrak{g}$. That is, the connected centralizer $C_{G}(A)^{\circ}$ is unipotent. There is a homomorphism $\check{\lambda}: k^{\times} \rightarrow G$, such that $\operatorname{Ad}(\check{\lambda}(t)) A=t A$ for all $t \in k^{*}$. This gives a grading

$$
\mathfrak{g}=\bigoplus_{j=-a}^{a} \mathfrak{g}(j),
$$

where $\mathfrak{g}(j)=\left\{x \in \mathfrak{g}: \lambda(t) x=t^{j} \cdot x \forall t \in k^{\times}\right\}$and $a=\max \{j: \mathfrak{g}(j) \neq 0\}$. Since $A$ is distinguished the linear map $\operatorname{ad}(A): \mathfrak{g}(0) \rightarrow \mathfrak{g}(1)$ is a bijection.

Set $m=a+1$, assume this is nonzero in $k$, and choose a root of unity $\zeta \in k^{\times}$of order $m$. The inner automorphism $\theta_{A}:=\operatorname{Ad}(\check{\lambda}(\zeta)) \in \operatorname{Aut}(\mathfrak{g})^{\circ}$ has order $m$, giving rise to a $\mathbb{Z} / m$-grading

$$
\mathfrak{g}=\bigoplus_{i \in \mathbb{Z} / m} \mathfrak{g}_{i}
$$

where $\mathfrak{g}_{i}$ is the $\zeta^{i}$-eigenspace of $\theta_{A}$ in $\mathfrak{g}$. We have

$$
\mathfrak{g}_{i}=\sum_{\substack{-a \leq j \leq a \\ j \equiv i}} \mathfrak{g}(j),
$$

so that

$$
\mathfrak{g}_{0}=\mathfrak{g}(0) \quad \text { and } \quad \mathfrak{g}_{1}=\mathfrak{g}(-a) \oplus \mathfrak{g}(1)
$$

Choose a maximal torus $T$ in a Borel subgroup $B$ of $G$ such that $\check{\lambda} \in X_{*}(T)$ and $\langle\alpha, \check{\lambda}\rangle \geq 0$ for all roots $\alpha$ of $T$ in $B$. For each of the simple roots $\alpha_{1}, \ldots, \alpha_{\ell}$ we have $\left\langle\alpha_{i}, \check{\lambda}\right\rangle \in\{0,1\}$. We set $s_{i}=\left\langle\alpha_{i}, \check{\lambda}\right\rangle$, and also put $s_{0}=1$. Since $\mathfrak{g}(-a)$ contains the lowest root space, it follows that $\left(s_{0}, s_{1}, \ldots, s_{\ell}\right)$ are the normalized Kac-coordinates of $\theta_{A}$.

Proposition 8.1 The following are equivalent.

1. There exists $M \in \mathfrak{g}(-a)$ such that $M+A$ is regular semisimple.

2. There exists $M \in \mathfrak{g}(-a)$ such that $M+A$ is semisimple.

3. The automorphism $\theta_{A}$ is stable.

Proof: Implication $1 \Rightarrow 2$ is obvious.

We prove $2 \Rightarrow 3$. Since $A$ is distinguished, the centralizer $C_{G_{0}}(A)$ is finite. Since $G_{0}$ preserves each summand $\mathfrak{g}(j)$, we have $C_{G_{0}}(M+A) \subset C_{G_{0}}(A)$. Hence $C_{G_{0}}(M+A)$ is also finite, so the $G_{0}$-orbit of $M+A$ in $\mathfrak{g}_{1}$ is stable. 
The implication $3 \Rightarrow 1$ is proved in [29, 9.5]. We give Springer's argument here for completeness. Let $F$ be a $G$-invariant polynomial on $\mathfrak{g}$ such that $F(x) \neq 0$ if and only if $x$ is regular semisimple. For example, we can choose $F$ corresponding, under the Chevalley isomorphism $k[\mathfrak{t}]^{G} \stackrel{\sim}{\rightarrow} k[\mathfrak{t}]^{W}$, to the product of the roots. Now assuming that 3 holds, there are vectors $Z \in \mathfrak{g}(-a)$ and $Y_{0} \in \mathfrak{g}(1)$ such that $Z+Y_{0}$ is semisimple and has finite stabilizer in $G_{0}$. The centralizer $\mathfrak{m}=\mathfrak{z}\left(Z+Y_{0}\right)$ is then reductive, with $\mathfrak{m}^{\theta}=0$, so $\mathfrak{m}$ is a Cartan subalgebra of $\mathfrak{g}$ and $Z+Y_{0}$ is in fact regular semisimple. Hence the polynomial $F_{Z}$ on $\mathfrak{g}(1)$ given by $F_{Z}(Y):=F(Z+Y)$ does not vanish identically. Since $A$ is distinguished, the orbit $\operatorname{Ad}\left(G_{0}\right) A$ is dense in $\mathfrak{g}(1)$, so there is $g \in G_{0}$ such that $F_{Z}(\operatorname{Ad}(g) A)=$ $F\left(\operatorname{Ad}(g)^{-1} Z+A\right) \neq 0$. It follows that $\operatorname{Ad}(g)^{-1} Z+A$ is regular semisimple so 1 holds.

We say that a distinguished nilpotent element $A \in \mathfrak{g}$ is $S$-distinguished if the equivalent conditions of Prop. 8.1 hold.

A non-example: It can happen that $\mathfrak{g}(-a)+\mathfrak{g}(1)$ contains semisimple elements, but none have the form $M+A$ with $M \in \mathfrak{g}(-a)$. For example, suppose $\mathfrak{g}=\mathfrak{s p}_{6}$ and $A$ has Jordan blocks $(4,2)$. The automorphism $\theta_{A}$ has Kac coordinates

$$
1 \Rightarrow 1 \quad 0 \Leftarrow 1
$$

and has rank equal to 1 . It corresponds to $w \in W\left(C_{3}\right)$ of type $C_{2} \times C_{1}$, which is not $\mathbb{Z}$-regular, so $A$ is not $S$-distinguished.

Proposition 8.2 Assume that $\mathfrak{g}$ is of exceptional type and that $\theta \in \operatorname{Aut}(\mathfrak{g})^{\circ}$ is a stable inner automorphism whose Kac coordinates satisfy $s_{0}=1$. Then $\theta=\theta_{A}$ where $A$ is an S-distinguished nilpotent element in $\mathfrak{g}$.

Proof: In the tables of section 8.1 we have listed, for each $\theta$ with $s_{0}=1$, the conjugacy class of a nilpotent element $A$ such that $\theta_{A}$ has the normalized Kac coordinates of $\theta$.

Remark 1: For $n$ even there is a unique $S$-distinguished non-regular nilpotent class in $\mathfrak{s o}_{2 n}$ which is also $S$-distinguished in $\mathfrak{s o}_{2 n+1}$, having Jordan partitions $[2 n+1,2 n-1]$ and $[2 n+1,2 n-1,1]$, respectively. For $A$ in these classes $\theta_{A}$ has order $n$. In these and the exceptional cases, the map $A \mapsto \theta_{A}$ is a bijection from the set of $S$-distinguished nilpotent $G$-orbits in $\mathfrak{g}$ to the set of inner gradings on $\mathfrak{g}$ with $s_{0}=1$. However, Prop. 8.1 is false for $C_{n}, n \geq 2$.

Remark 2: If $A$ is $S$-distinguished then $\mathfrak{z}(M+A)$ is a canonical Cartan subalgebra for $\theta_{A}$ on which $\theta_{A}$ acts by an element of the conjugacy class in $W$ associated to $A$ via the Kazhdan-Lusztig map [13]. This follows from the argument in [13, 9.11], and confirms two entries in [28, Table 1] (for $\left.A=E_{8}\left(a_{6}\right), E_{8}\left(a_{7}\right)\right)$, listed there as conjectural.

Remark 3: There are exactly three cases where $\mathfrak{g}_{0}$ is a maximal proper Levi subalgebra in $\mathfrak{g}$. These occur in $G_{2}, F_{4}$ and $E_{8}$, for $a=2,3,5$ respectively, where $C_{G_{0}}(A)$ is a symmetric group $S_{3}, S_{4}, S_{5}$. These groups act irreducibly on the subspaces $\mathfrak{g}(-a)$ of dimensions $1,2,4$, in which the stabilizers of a vector in general position are the isotropy groups $S_{0}=\boldsymbol{\mu}_{3}, \boldsymbol{\mu}_{2} \times \boldsymbol{\mu}_{2}, 1$. These are the maximal abelian normal subgroups of $C_{G_{0}}(A)$. 


\section{Positive rank gradings for type $E_{6,7,8}$ (inner case)}

Assume now that $\mathfrak{g}$ has type $E_{n}$, for $n=6,7,8$. From Prop. 4.3 we have the following algorithm to find all inner automorphisms of $\mathfrak{g}$ having positive rank. For each $m \geq 1$ list the $W$-conjugacy classes of $m$-admissible elements in $W$. For a representative $w$ of each class, form the list $\operatorname{Kac}(w)_{\text {un }}$ and apply the normalization algorithm to each element of $\operatorname{Kac}(w)_{\text {un }}$, discarding duplicate results, to obtain the list $\operatorname{Kac}(w)$ of normalized Kac coordinates. Then by Prop. 4.3, the union of the lists $\operatorname{Kac}(w)$ over all conjugacy-classes of $m$-admissible $w$ gives all positive rank inner automorphisms of order $m$.

To find the $\operatorname{Kac}(w)_{\text {un }}$ when each $w_{i}$ is $\mathbb{Z}$-regular, we can use Prop. 5.1 to find the Kac coordinates of each $w_{i}$, which lead to those of $w$ via the normalization algorithm. It turns out that we obtain all positive rank gradings from those $m$-admissible $w$ for which each factor $w_{i}$ is not only elliptic but also $\mathbb{Z}$-regular in $W_{J_{i}}$. However, we do not have an a priori proof of this fact, so we must also compute Kac coordinates of lifts in the small number of cases where not all $w_{i}$ are $\mathbb{Z}$-regular.

These non-regular cases are handled as follows. By induction, we assume $w=w_{i}$ lies in no proper reflection subgroup and we consider the powers of $w$. To illustrate the method, take the nonregular element $w=E_{8}\left(a_{7}\right)=-A_{2} E_{6}$ of order 12. First list the 32 normalized Kac coordinates $\left(s_{i}\right)$ with $s_{i} \in\{0,1\}$ and $s_{0}+2 s_{1}+3 s_{2}+4 s_{3}+6 s_{4}+5 s_{5}+4 s_{6}+3 s_{7}+2 s_{8}=12$. We have $w^{2}$ and $w^{3}$ in the

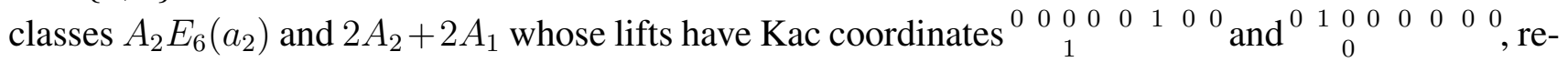

spectively. Only one of the 32 elements on the list satisfies these two conditions, namely $\left.\begin{array}{cccccccc}0 & 1 & 0 & 1 & 0 & 0 & 1 & 1\end{array}\right]$. Therefore this is the Kac diagram for the lift of $w$ in the class $E_{8}\left(a_{7}\right)$.

\subsection{A preliminary list of Kac coordinates for positive rank gradings of inner type}

For each possible order $m$ we list the $m$-admissible elements in $W\left(E_{6,7,8}\right)$, the rank $r=\operatorname{rank}(w)$. and the form of the un-normalized Kac-coordinates of the lifts of $w$ In the column $\operatorname{Kac}(w)_{\mathrm{un}}$, each $*$ is an independent variable integer ranging over a set of representatives of $\mathbb{Z} / m$ such that the order is always $m$. For each vector of $*$-values we apply the normalization algorithm to obtain the normalized $\operatorname{Kac}$ coordinates $\operatorname{Kac}(w)$ in the last column. The sets $\operatorname{Kac}(w)$ are not disjoint. In a second set of tables (section 9.2), we will select, for each $\theta$ appearing in $\cup_{w} \operatorname{Kac}(w)$, a $w$ of maximal rank for which $\operatorname{Kac}(w)$ contains $\theta$.

We use Carter's notation for conjugacy classes in $W$, augmented as follows. If $X$ is a conjugacy class and $-1 \in W$ then $-X=\{-w: w \in X\}$. This makes some classes easier to understand; for example, $E_{8}\left(a_{7}\right)=-A_{2} E_{6}$. 
Table 16: $\operatorname{Kac}(w)_{\text {un }}$ and $\operatorname{Kac}(w)$ for $m$-admissible $w$ in $W\left(E_{6}\right)$

\begin{tabular}{|c|c|c|c|c|c|}
\hline$m$ & $w$ & $r$ & & & \\
\hline 12 & $E_{6}$ & 1 & & & $\begin{array}{l}1 \\
1 \\
1\end{array}$ \\
\hline 9 & $E_{6}\left(a_{1}\right)$ & 1 & & & $\begin{array}{l}0 \\
1 \\
1\end{array}$ \\
\hline 8 & $D_{5}$ & 1 & & & $\begin{array}{l}1 \\
1 \\
1\end{array}$ \\
\hline 6 & $E_{6}\left(a_{2}\right)$ & 2 & & & $\begin{array}{l}1 \\
0 \\
1\end{array}$ \\
\hline 6 & $A_{5}$ & 1 & & & $\begin{array}{l}1 \\
* \\
*\end{array}$ \\
\hline 6 & $D_{4}$ & 1 & & & $\begin{array}{l}1 \\
1 \\
*\end{array}$ \\
\hline 5 & $A_{4}$ & 1 & & & $\begin{array}{l}1 \\
* \\
*\end{array}$ \\
\hline 4 & $D_{4}\left(a_{1}\right)$ & 2 & & & $\begin{array}{l}0 \\
1 \\
*\end{array}$ \\
\hline 4 & $A_{3}$ & 1 & & & $\begin{array}{l}1 \\
* \\
*\end{array}$ \\
\hline 3 & $3 A_{2}$ & 3 & & & $\begin{array}{l}* \\
1 \\
1\end{array}$ \\
\hline 3 & $2 A_{2}$ & 2 & & & $\begin{array}{l}* \\
* \\
*\end{array}$ \\
\hline 3 & $A_{2}$ & 1 & & & $\begin{array}{l}1 \\
1 \\
*\end{array}$ \\
\hline 2 & $4 A_{1}$ & 4 & & & $\begin{array}{l}1 \\
* \\
1\end{array}$ \\
\hline 2 & $3 A_{1}$ & 3 & & & $\begin{array}{l}1 \\
* \\
* \\
*\end{array}$ \\
\hline 2 & $2 A_{1}$ & 2 & & & $\begin{array}{l}1 \\
* \\
1\end{array}$ \\
\hline 2 & $A_{1}$ & 1 & & & \\
\hline
\end{tabular}


Table 17: $\operatorname{Kac}(w)_{\text {un }}$ and $\operatorname{Kac}(w)$ for $m$-admissible $w$ in $W\left(E_{7}\right)$

\begin{tabular}{|c|c|c|c|c|c|c|c|c|}
\hline$m$ & $w$ & $r$ & $\operatorname{Kac}(w)_{\mathrm{un}}$ & $\operatorname{Kac}(w)$ & & & & \\
\hline 18 & $E_{7}$ & 1 & $\begin{array}{|lllllll|} & 1 & 1 & 1 & 1 & 1 & 1 \\
& & & 1 & & & \\
\end{array}$ & $\begin{array}{|lllllll|}1 & 1 & 1 & 1 & 1 & 1 & 1 \\
& & & 1 & & & \\
\end{array}$ & & & & \\
\hline 14 & $E_{7}\left(a_{1}\right)$ & 1 & $\begin{array}{|lllllll|} & 1 & 1 & 0 & 1 & 1 & 1 \\
& & & 1 & & & \\
\end{array}$ & $\begin{array}{|lllllll|}1 & 1 & 1 & 0 & 1 & 1 & 1 \\
& & & 1 & & & \\
\end{array}$ & & & & \\
\hline 12 & $E_{7}\left(a_{2}\right)$ & 1 & $\begin{array}{|lllllll|}1 & 1 & 0 & 1 & 0 & 1 & 1 \\
& & & 1 & & & \\
\end{array}$ & \begin{tabular}{lllllll|}
1 & 1 & 0 & 1 & 0 & 1 & 1 \\
& & & 1 & & &
\end{tabular} & & & & \\
\hline 12 & $E_{6}$ & 1 & \begin{tabular}{|llllll}
$*$ & 1 & 1 & 1 & 1 & 1
\end{tabular} & $\begin{array}{|lllllll|}1 & 0 & 1 & 0 & 1 & 1 & 1 \\
& & & & & \\
\end{array}$ & 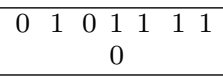 & $\begin{array}{lllllll}1 & 1 & 0 & 1 & 0 & 1 & 1 \\
& & & 1 & & & \\
\end{array}$ & & \\
\hline 10 & $D_{6}$ & 1 & \begin{tabular}{|c|cccccc|}
$* *$ & $*$ & 1 & 1 & 1 & 1 \\
& & 1 & & & \\
\end{tabular} & $\begin{array}{|llllllll|}0 & 1 & 0 & 1 & 0 & 1 & 0 \\
& & & 1 & & & \\
\end{array}$ & $\begin{array}{ccccccc}1 & 0 & 1 & 0 & 1 & 0 & 1 \\
& & & 1 & & & \\
\end{array}$ & $\begin{array}{lllllll}1 & 1 & 0 & 1 & 0 & 1 & 1 \\
& & & 0 & & & \\
\end{array}$ & & \\
\hline 9 & $E_{6}\left(a_{1}\right)$ & 1 & $\begin{array}{|cccccc|}* & 1 & 1 & 0 & 1 & 1\end{array}$ & $\begin{array}{|lllllll|}0 & 1 & 0 & 1 & 0 & 1 & 1 \\
& & & 0 & & & \\
\end{array}$ & $\begin{array}{lllllll}1 & 0 & 1 & 0 & 0 & 1 & 1 \\
& & & 1 & & & \\
\end{array}$ & & & \\
\hline 8 & $D_{5}$ & 1 & $\begin{array}{llllll}* * & * & 1 & 1 & 1 & * \\
& & 1 & & & \end{array}$ & 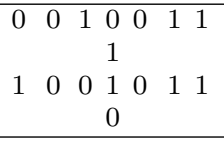 & $\begin{array}{ccccccc} & 1 & 0 & 0 & 1 & 1 & 1 \\
& & & 0 & & & \\
1 & 0 & 1 & 0 & 1 & 0 & 1 \\
& & & 0 & & & \\
\end{array}$ & $\begin{array}{ccccccc}0 & 1 & 0 & 1 & 0 & 1 & 0 \\
& & & 0 & & & \\
1 & 0 & 0 & 1 & 0 & 0 & 1 \\
& & & & 1 & & \\
\end{array}$ & 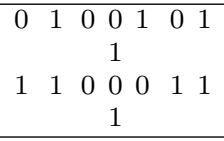 & \\
\hline 8 & $D_{6}\left(a_{1}\right)$ & 1 & $\begin{array}{|lllllll|}* & * & 1 & 0 & 1 & 1 & 1 \\
& & 1 & & & \\
\end{array}$ & $\begin{array}{|lllllll|}0 & 1 & 0 & 0 & 1 & 0 & 1 \\
& & & 1 & & & \\
\end{array}$ & $\begin{array}{lllllll}1 & 0 & 0 & 1 & 0 & 1 & 1 \\
& & & 0 & & & \\
\end{array}$ & & & \\
\hline 8 & $A_{7}$ & 1 & $\begin{array}{|lllllll|}1 & 1 & 1 & 1 & 1 & 1 & 1 \\
& & & * & & & \\
\end{array}$ & $\begin{array}{|lllllll|}0 & 1 & 0 & 1 & 0 & 1 & 0 \\
& & & 0 & & & \\
\end{array}$ & & & & \\
\hline 7 & $A_{6}$ & 1 & \begin{tabular}{ccccccc|}
$*$ & 1 & 1 & 1 & 1 & 1 & 1 \\
& & & $*$ & & &
\end{tabular} & $\begin{array}{|ccccccc|}0 & 1 & 0 & 1 & 0 & 0 & 1 \\
& & & 0 & & & \end{array}$ & & & & \\
\hline 6 & $E_{7}\left(a_{4}\right)$ & 3 & \begin{tabular}{|lllllll}
1 & 0 & 0 & 1 & 0 & 0 & 1 \\
& & & 0 & & & \\
\end{tabular} & $\begin{array}{|lllllll|}1 & 0 & 0 & 1 & 0 & 0 & 1 \\
& & & 0 & & & \\
\end{array}$ & & & & \\
\hline 6 & $D_{6}\left(a_{2}\right)$ & 2 & $\begin{array}{|lllllll|}* & * & 1 & 0 & 1 & 0 & 1 \\
& & & & & & \\
\end{array}$ & $\begin{array}{|lllllll|}0 & 1 & 0 & 0 & 0 & 1 & 0 \\
& & & 1 & & & \\
\end{array}$ & $\begin{array}{lllllll}1 & 0 & 0 & 1 & 0 & 0 & 1 \\
& & & 0 & & & \\
\end{array}$ & & & \\
\hline 6 & $E_{6}\left(a_{2}\right)$ & 2 & \begin{tabular}{|llllll}
$*$ & 1 & 0 & 1 & 0 & 1
\end{tabular} & $\begin{array}{|lllllll|}0 & 1 & 0 & 0 & 1 & 0 & 1 \\
& & & 0 & & & \\
\end{array}$ & $\begin{array}{lllllll}1 & 0 & 0 & 1 & 0 & 0 & 1 \\
& & & 0 & & & \\
\end{array}$ & & & \\
\hline 6 & $D_{4}$ & 1 & 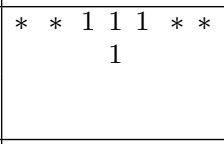 & $\begin{array}{ccccccc}1 & 0 & 0 & 0 & 1 & 0 & 0 \\
& & & 1 & & & \\
0 & 1 & 0 & 0 & 1 & 0 & 1 \\
& & & 0 & & & \\
\end{array}$ & $\begin{array}{ccccccc}0 & 0 & 0 & 0 & 1 & 1 & 1 \\
& & & 0 & & & \\
0 & 1 & 0 & 0 & 0 & 1 & 0 \\
& & & & 1 & & \\
\end{array}$ & $\begin{array}{ccccccc}0 & 0 & 1 & 0 & 0 & 1 & 1 \\
& & & 0 & & & \\
1 & 0 & 0 & 1 & 0 & 0 & 1 \\
& & & 0 & & & \\
\end{array}$ & 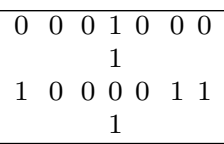 & 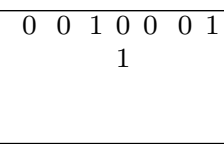 \\
\hline 6 & $A_{5}^{\prime \prime}$ & 1 & \begin{tabular}{|cccccc}
$*$ & 1 & 1 & 1 & 1 & 1
\end{tabular} & \begin{tabular}{|llllllll}
0 & 0 & 0 & 1 & 0 & 1 & 0 \\
& & 0 & & & \\
\end{tabular} & 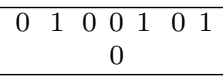 & 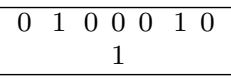 & $\begin{array}{lllllll} & 0 & 0 & 1 & 0 & 0 & 1 \\
& & & 0 & & & \\
\end{array}$ & \\
\hline 6 & $A_{5}^{\prime}$ & 1 & \begin{tabular}{|cccc}
1 & 1 & 1 & 1 \\
& & & 1
\end{tabular} & $\begin{array}{|llllllll|} & 0 & 0 & 1 & 0 & 1 & 0 & 0 \\
& & & 0 & & & \\
\end{array}$ & 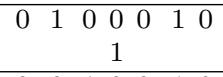 & $\begin{array}{lllllll}1 & 0 & 0 & 1 & 0 & 0 & 1 \\
& & & 0 & & & \\
\end{array}$ & $\begin{array}{lllllll}1 & 1 & 0 & 0 & 0 & 1 & 1 \\
& & & 0 & & & \\
\end{array}$ & \\
\hline 5 & $A_{4}$ & 1 & \begin{tabular}{|ccccc}
$* * * 1$ & 1 & 1 & 1 & $*$ \\
& $*$ & & & \\
\end{tabular} & \begin{tabular}{|llllllll}
0 & 0 & 0 & 1 & 0 & 0 & 1 \\
& & 0 & & & \\
\end{tabular} & 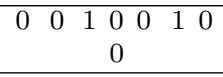 & $\begin{array}{lllllll}0 & 1 & 0 & 0 & 0 & 1 & 1 \\
& & & 0 & & & \\
\end{array}$ & 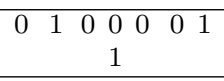 & $\begin{array}{ccccccc}1 & 0 & 0 & 0 & 1 & 0 & 1 \\
& & 0 & & & \\
\end{array}$ \\
\hline 4 & $2 A_{3}$ & 2 & \begin{tabular}{|ccccccc}
1 & 1 & 1 & $*$ & 1 & 1 & 1 \\
& & $*$ & & \\
\end{tabular} & $\begin{array}{|llllllll|}0 & 0 & 0 & 1 & 0 & 0 & 0 \\
& & 0 & & & \\
\end{array}$ & 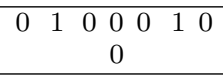 & & & \\
\hline 4 & $D_{4}\left(a_{1}\right)$ & 2 & $\begin{array}{|cccc|}* * 1 & 0 & 1 & * * \\
& 1 & & \\
\end{array}$ & \begin{tabular}{|llllllll}
0 & 0 & 0 & 0 & 1 & 0 & 1 \\
& & 0 & & & \\
\end{tabular} & 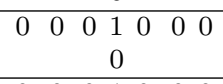 & \begin{tabular}{ccccccc}
0 & 0 & 1 & 0 & 0 & 0 & 1 \\
& \multicolumn{7}{c}{} & 0 & & \\
\end{tabular} & 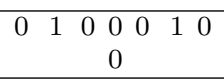 & $\begin{array}{lllllll}1 & 0 & 0 & 0 & 0 & 0 & 1 \\
& & & 1 & & \\
\end{array}$ \\
\hline 4 & $A_{3}$ & 1 & $\begin{array}{cccccc}* * & * & 1 & 1 & 1 & * \\
& & & * & & \\
\end{array}$ & \begin{tabular}{|cccccccc}
0 & 0 & 0 & 0 & 1 & 0 & 1 \\
& & & 0 & & & \\
0 & 1 & 0 & 0 & 0 & 1 & 0 \\
& & & 0 & & & \\
\end{tabular} & $\begin{array}{ccccccc}0 & 0 & 0 & 1 & 0 & 0 & 0 \\
& & & 0 & & & \\
1 & 0 & 0 & 0 & 0 & 1 & 1 \\
& & & & 0 & & \\
\end{array}$ & $\begin{array}{ccccccc}0 & 0 & 1 & 0 & 0 & 0 & 1 \\
& & & 0 & & & \\
1 & 0 & 0 & 0 & 0 & 0 & 1 \\
& & & & 1 & & \\
\end{array}$ & $\begin{array}{ccccccc}0 & 0 & 0 & 0 & 0 & 1 & 0 \\
& & & & 1 & & \\
\end{array}$ & \\
\hline 3 & $3 A_{2}$ & 3 & 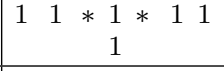 & \begin{tabular}{|lllllll}
0 & 0 & 0 & 0 & 1 & 0 & 0 \\
& & & 0 & & & \\
\end{tabular} & & & & \\
\hline 3 & $2 A_{2}$ & 2 & $\begin{array}{|ccc|}1 & 1 & * 1 * * * \\
& & 1 \\
\end{array}$ & \begin{tabular}{|llllllll}
0 & 0 & 0 & 0 & 1 & 0 & 0 \\
& & 0 & & & \\
\end{tabular} & 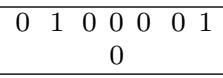 & & & \\
\hline 3 & $A_{2}$ & 1 & $\begin{array}{c}* * * 1 * * * \\
1 \\
\end{array}$ & $\begin{array}{|lllllll|}0 & 0 & 0 & 0 & 0 & 1 & 1 \\
& 0 & & & \\
\end{array}$ & 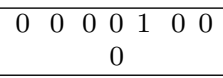 & 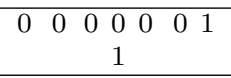 & $\begin{array}{ccccccc}0 & 1 & 0 & 0 & 0 & 0 & 1 \\
& & & 0 & & & \\
\end{array}$ & \\
\hline 2 & $7 A_{1}$ & 7 & $\begin{array}{cccccccc}0 & 0 & 0 & 0 & 0 & 0 & 0 \\
& & & 1 & & & \end{array}$ & $\begin{array}{|ccccccc|}0 & 0 & 0 & 0 & 0 & 0 & 0 \\
& & & 1 & & & \end{array}$ & & & & \\
\hline 2 & $6 A_{1}$ & 6 & 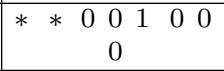 & $\begin{array}{|lllllll|} & 0 & 0 & 0 & 0 & 0 & 0 \\
& & & 1 & & & \\
\end{array}$ & & & & \\
\hline 2 & $5 A_{1}$ & 5 & 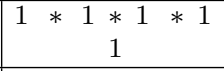 & $\begin{array}{|lllllll|}0 & 0 & 0 & 0 & 0 & 0 & 0 \\
& & & 1 & & & \\
\end{array}$ & & & & \\
\hline 2 & $4 A_{1}^{\prime}$ & 4 & 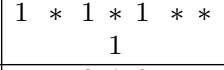 & \begin{tabular}{|lllllll}
0 & 0 & 0 & 0 & 0 & 0 & 0 \\
& & & & 1 & & \\
\end{tabular} & & & & \\
\hline 2 & $4 A_{1}^{\prime \prime}$ & 4 & $\begin{array}{lllll}* & * & 0 & 1 & 0 \\
& & 0 & * & * \\
\end{array}$ & $\begin{array}{|llllllll|} & 0 & 0 & 0 & 0 & 0 & 0 \\
& & & 1 & & & \\
\end{array}$ & 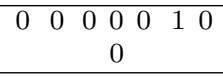 & & & \\
\hline 2 & $3 A_{1}^{\prime}$ & 3 & $\begin{array}{c}1 * 1 * * * * \\
1 \\
\end{array}$ & \begin{tabular}{|llllllll}
0 & 0 & 0 & 0 & 0 & 0 & 0 \\
& & & 1 & & & \\
\end{tabular} & $\begin{array}{ccccccc} & 0 & 0 & 0 & 0 & 0 & 1 \\
& & 0 & & & \\
\end{array}$ & & & \\
\hline 2 & $3 A_{1}^{\prime \prime}$ & 3 & $\begin{array}{c}1 * * * 1 * * \\
1 \\
\end{array}$ & \begin{tabular}{|llllllll}
0 & 0 & 0 & 0 & 0 & 0 & 0 \\
& & & 1 & & & \\
\end{tabular} & 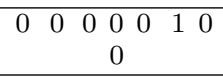 & & & \\
\hline 2 & $2 A_{1}$ & 2 & \begin{tabular}{ccc|}
$* * 1$ & $* 1$ & $* *$ \\
& $*$ & \\
\end{tabular} & $\begin{array}{|lllllll|}0 & 0 & 0 & 0 & 0 & 0 & 0 \\
& & & 1 & & & \\
\end{array}$ & $\begin{array}{cccccc} & 45^{0} & 0 & 0 & 1 & 0 \\
& 0 & & & \\
\end{array}$ & $\begin{array}{ccccccc}1 & 0 & 0 & 0 & 0 & 0 & 1 \\
& & & 0 & & & \\
\end{array}$ & & \\
\hline 2 & $A_{1}$ & 1 & $\begin{array}{c}* * * * 1 * * \\
*\end{array}$ & $\begin{array}{|llllllll|}0 & 0 & 0 & 0 & 0 & 0 & 0 \\
& & & 1 & & & \\
\end{array}$ & 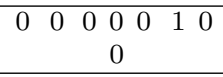 & $\begin{array}{ccccccc}1 & 0 & 0 & 0 & 0 & 0 & 1 \\
& & & 0 & & & \end{array}$ & & \\
\hline
\end{tabular}


Table 18: $\operatorname{Kac}(w)_{\text {un }}$ and $\operatorname{Kac}(w)$ for $m$-admissible $w$ in $W\left(E_{8}\right)$

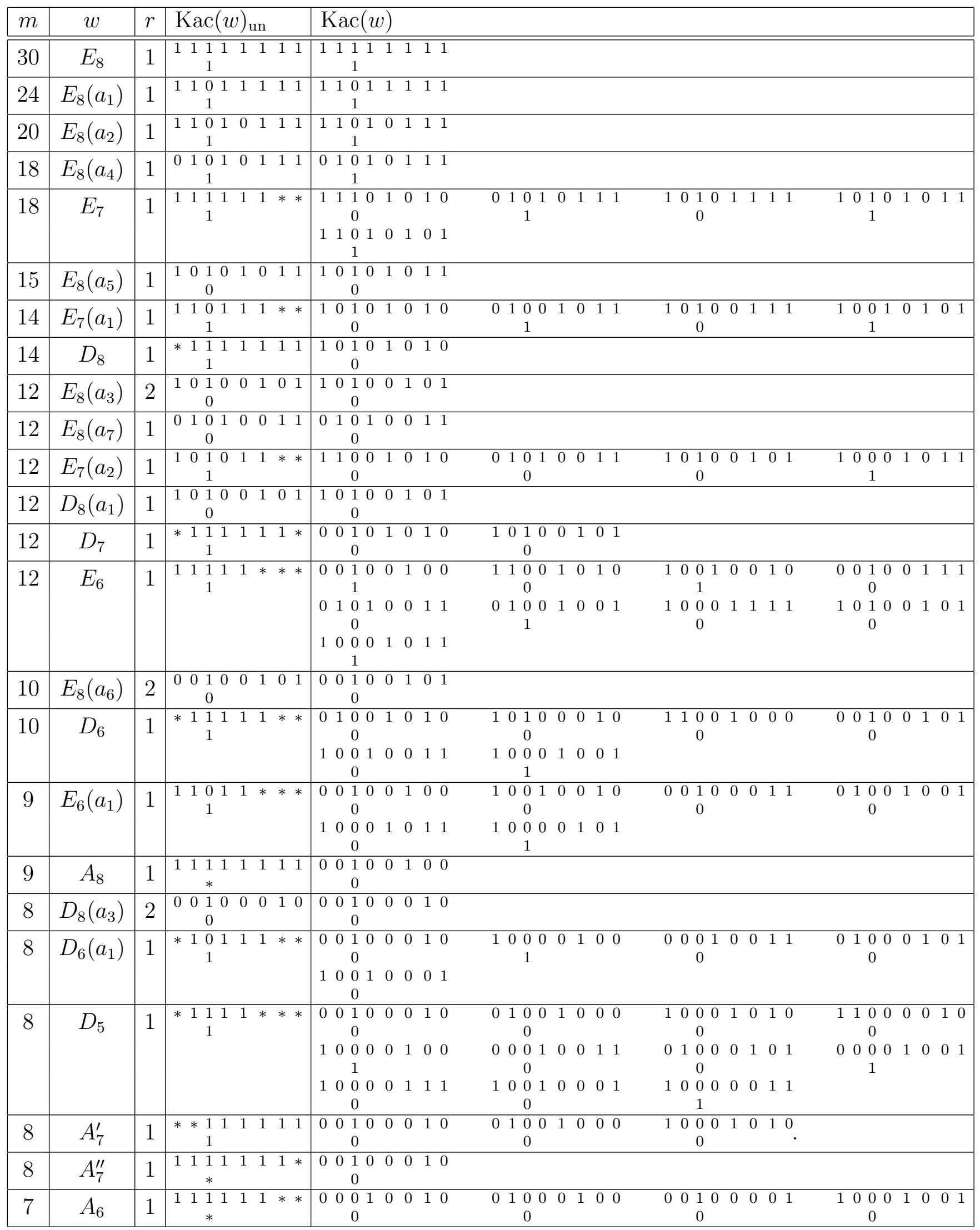


Table 18 continued: $\operatorname{Kac}(w)_{\text {un }}$ and $\operatorname{Kac}(w)$ for $m$-admissible $w$ in $W\left(E_{8}\right)$

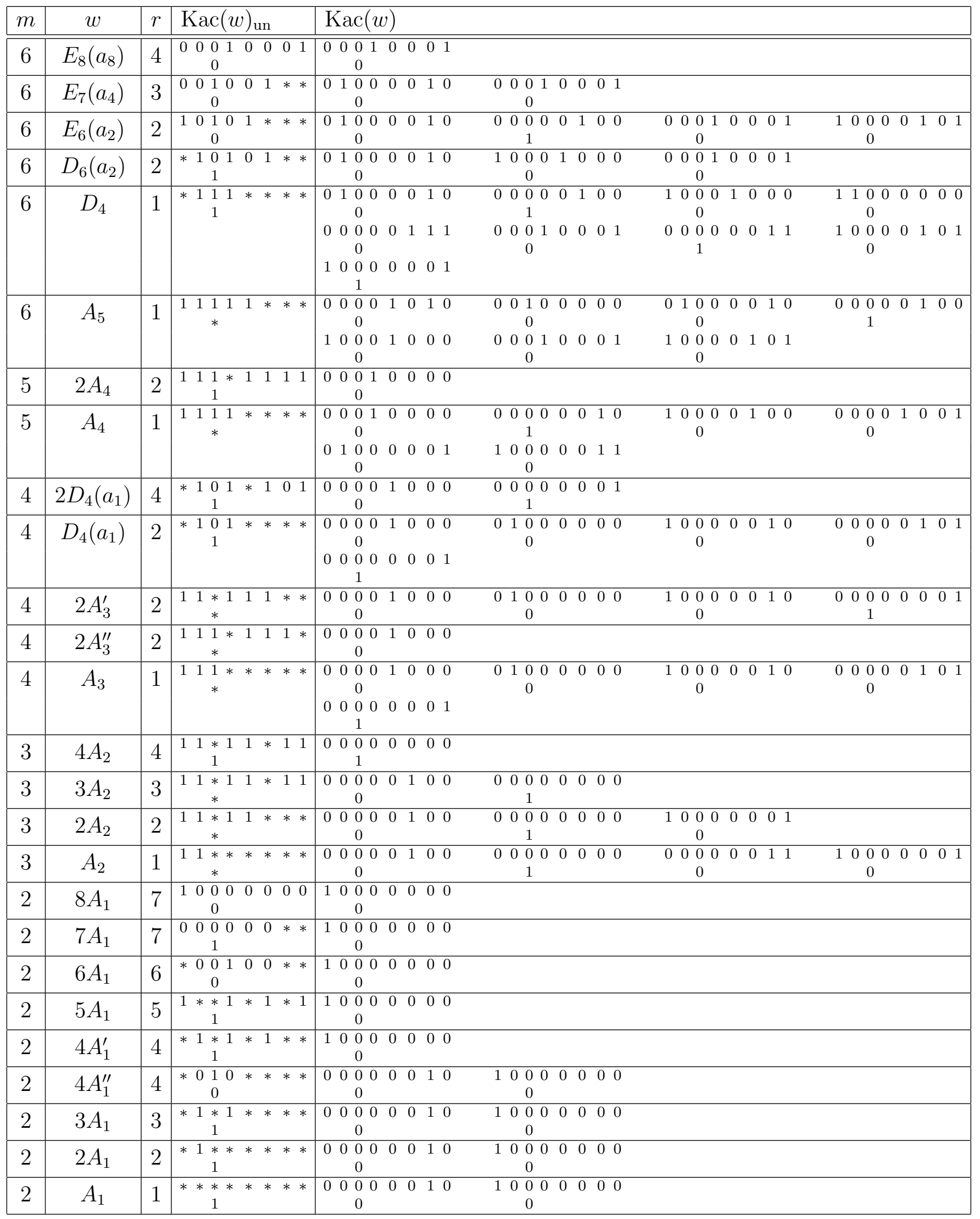




\subsection{Tables of positive rank gradings for $E_{6}, E_{7}$ and $E_{8}$}

The previous lists contain the Kac coordinates of all positive rank gradings, usually with multiple occurrences. We now discard those in each $\operatorname{Kac}(w)$ which appear in some $\operatorname{Kac}\left(w^{\prime}\right)$ with $\operatorname{rank}\left(w^{\prime}\right)>$ $\operatorname{rank}(w)$. The remaining elements of $\operatorname{Kac}(w)$ are then the Kac coordinates of automorphisms $\theta$ of order $m$ with $\operatorname{rank}(\theta)=\operatorname{rank}(w)$. For each grading $\theta$ there still may be more than one $w$ with $\operatorname{rank}(\theta)=\operatorname{rank}(w)$. It turns out that every $\theta$ of positive rank is contained in $\operatorname{Kac}(w)_{\text {un }}$ for some $m$ admissible $w$ which is a $\mathbb{Z}$-regular element in the Weyl group Levi of a Levi subgroup $L_{\theta}$ and $\theta$ is a principal inner automorphism of the Lie algebra of $L_{\theta}$. This Levi subgroup corresponds to the subset $J$ of Lemma 7.6 and is indicated in the right most column of the tables below. For example, in $E_{7}$ the Kac diagrams

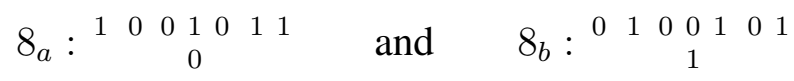

occur in $\operatorname{Kac}(w)$ for $w$ of types $D_{6}\left(a_{1}\right)$ and $D_{5}$. Since $D_{6}\left(a_{1}\right)$ is not regular in any Levi subgroup of $W\left(E_{7}\right)$ and $w=D_{5}$ is regular in the $D_{5}$ Levi subgroup, we choose $w=D_{5}$, discard $w=D_{6}\left(a_{1}\right)$, and set $L_{\theta}=D_{5}$.

Since $\theta$ is principal on the Lie algebra of $L_{\theta}$, there is a conjugate $\theta^{\prime}$ of $\theta$ whose un-normalized Kac diagram has a 1 on each node of $J$ (cf. Lemma 7.6). There may be more than one such $J$, corresponding to various conjugates $\theta^{\prime}$, and we just pick one of them.

In the tables we try to write $w$ in a form which exhibits its regularity in the Weyl group $W_{J}$. For example, in $E_{6}$ the gradings $4_{a}, 4_{b}$ have $w=D_{4}\left(a_{1}\right) .{ }^{1}$ In case $4_{a}$, which is stable, we give the alternate expression $w=E_{6}^{3}$ to make it clear that $w$ is $\mathbb{Z}$-regular in $W\left(E_{6}\right)$. In case $4_{b}$ there is no $W_{\text {aff }}(R)$ conjugate of $\theta$ with 1 's on the $E_{6}$ subdiagram. However, $w=D_{5}^{2}$ is the square of a Coxeter element in $W_{D_{5}}$, hence is $\mathbb{Z}$-regular in $W_{D_{5}}$.

The rows in our tables are ordered by decreasing $m$. The positive rank inner gradings of a given order $m$ are named $m_{a}, m_{b}, m_{c}, \ldots$, where $m_{a}$ is the unique principal grading of order $m$. The principal grading $m_{a}$ has maximal rank and minimal dimension of $\mathfrak{g}_{0}$ among all gradings of order $m$. The remaining rows of order $m$ are grouped according to $w$ and $L_{\theta}$, ordered in each group by increasing dimension of $\mathfrak{g}_{0}$.

The little Weyl groups $W(\mathfrak{c}, \theta)$ are also given, along with their degrees. These are either cyclic or given by their notation in [27]. We explain their computation in section 10 .

\footnotetext{
${ }^{1}$ cf. Panyushev, Example 4.5.
} 
Table 19: The gradings of positive rank in type $E_{6}$ (inner case)

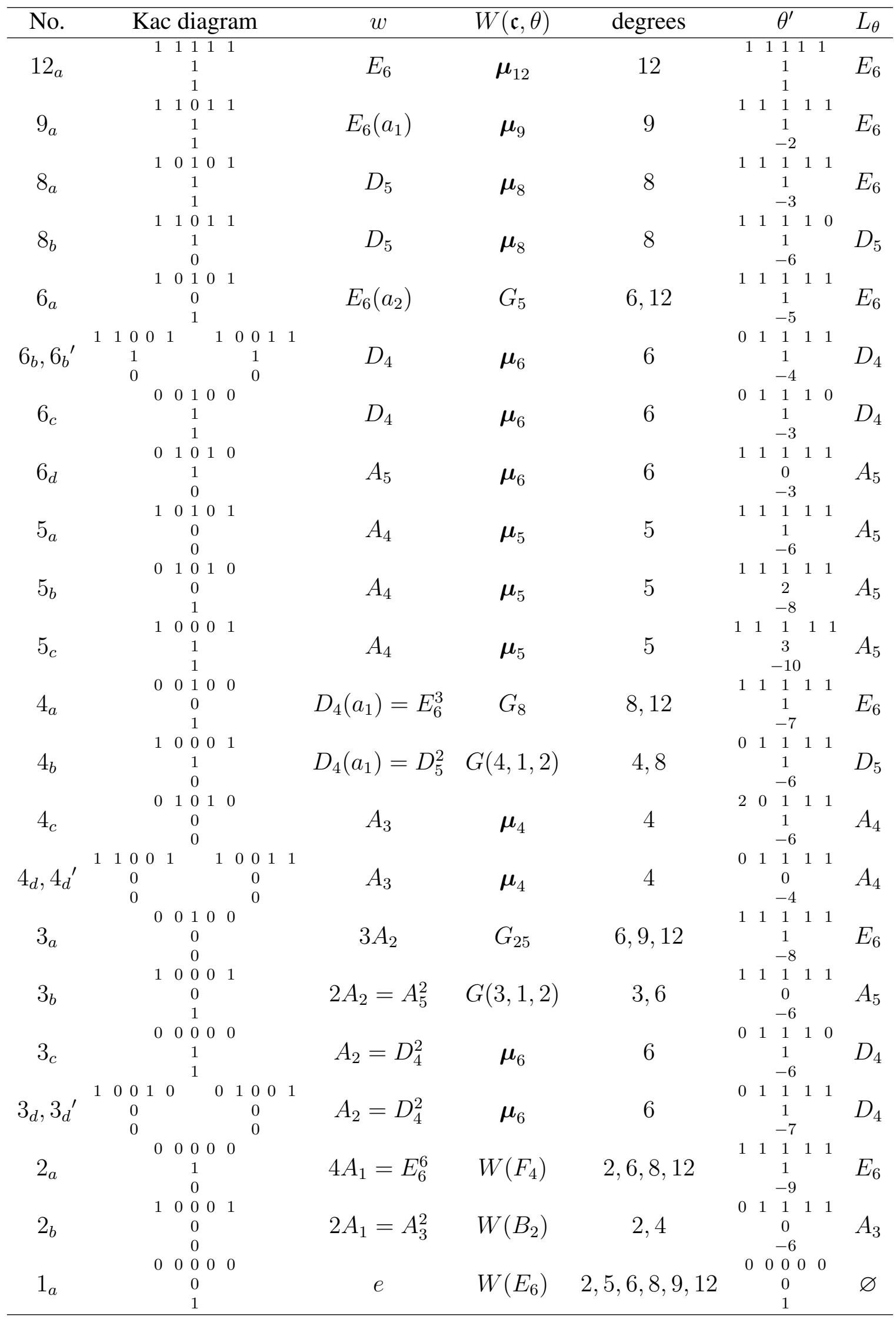


Table 20: The gradings of positive rank in type $E_{7}$

\begin{tabular}{|c|c|c|c|c|c|c|}
\hline No. & Kac diagram & $w$ & $W(\mathfrak{c}, \theta)$ & degrees & $\theta^{\prime}$ & $L_{\theta}$ \\
\hline $18_{a}$ & 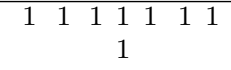 & $E_{7}$ & $\boldsymbol{\mu}_{18}$ & 18 & $\begin{array}{lllllll} & 1 & 1 & 1 & 1 & 1 & 1 \\
& & & 1 & & & \\
\end{array}$ & $E_{7}$ \\
\hline $14_{a}$ & $\begin{array}{lllllll}1 & 1 & 1 & 0 & 1 & 1 & 1\end{array}$ & $E_{7}\left(a_{1}\right)=-A_{6}$ & $\boldsymbol{\mu}_{14}$ & 14 & $\begin{array}{lllllll}-3 & 1 & 1 & 1 & 1 & 1 & 1 \\
& & & 1 & & & \end{array}$ & $E_{7}$ \\
\hline $12_{a}$ & $\begin{array}{lllllll}1 & 1 & 1 & 0 & 1 & 0 & 1 \\
& & & 1 & & & \end{array}$ & $E_{6}$ & $\boldsymbol{\mu}_{12}$ & 12 & $\begin{array}{lllllll}-5 & 1 & 1 & 1 & 1 & 1 & 1 \\
& & & 1 & & & \end{array}$ & $E_{7}$ \\
\hline $12_{b}$ & $\begin{array}{lllllll}1 & 1 & 0 & 1 & 0 & 1 & 1 \\
& & & 1 & & & \end{array}$ & $E_{7}\left(a_{2}\right)=-E_{6}$ & $\boldsymbol{\mu}_{12}$ & 12 & $\begin{array}{llllll}-6 & 1 & 1 & 1 & 1 & 1\end{array}$ & $E_{6}$ \\
\hline $12_{c}$ & $\begin{array}{lllllll}0 & 1 & 0 & 1 & 1 & 1 & 1\end{array}$ & $E_{6}$ & $\boldsymbol{\mu}_{12}$ & 12 & $\begin{array}{lllllll}-4 & 1 & 1 & 1 & 1 & 1 & 0 \\
& & & 1 & & & \end{array}$ & $E_{6}$ \\
\hline $10_{a}$ & $\begin{array}{ccccccc}1 & 0 & 1 & 0 & 1 & 0 & 1 \\
& & & 1 & & & \end{array}$ & $D_{6}$ & $\boldsymbol{\mu}_{10}$ & 10 & 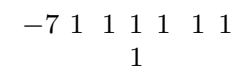 & $D_{6}$ \\
\hline $10_{b}$ & $\begin{array}{lllllll}1 & 1 & 0 & 1 & 0 & 1 & 1\end{array}$ & $D_{6}$ & $\boldsymbol{\mu}_{10}$ & 10 & 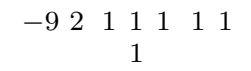 & $D_{6}$ \\
\hline $10_{c}$ & $\begin{array}{llllllll}0 & 1 & 0 & 1 & 0 & 1 & 0 \\
& & & & 1 & & & \end{array}$ & $D_{6}$ & $\boldsymbol{\mu}_{10}$ & 10 & 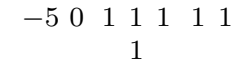 & $D_{6}$ \\
\hline $9_{a}$ & $\begin{array}{lllllll}0 & 1 & 0 & 1 & 0 & 1 & 1\end{array}$ & $E_{6}\left(a_{1}\right)=E_{7}^{2}$ & $\boldsymbol{\mu}_{18}$ & 18 & 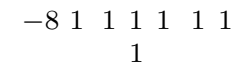 & $E_{7}$ \\
\hline $9_{b}$ & $\begin{array}{ccccccc}1 & 0 & 1 & 0 & 0 & 1 & 1 \\
& & & 1 & & & \end{array}$ & $E_{6}\left(a_{1}\right)$ & $\mu_{9}$ & 9 & $\begin{array}{lllllll}-7 & 1 & 1 & 1 & 1 & 1 & 0 \\
& & 1 & & \end{array}$ & $E_{6}$ \\
\hline $8_{a}$ & $\begin{array}{lllllll}1 & 0 & 0 & 1 & 0 & 1 & 1\end{array}$ & $D_{5}$ & $\boldsymbol{\mu}_{8}$ & 8 & $\begin{array}{lllllll}-9 & 1 & 1 & 1 & 1 & 1 & 1 \\
& & & 1 & & & \end{array}$ & $D_{5}$ \\
\hline $8_{b}$ & $\begin{array}{lllllll}0 & 1 & 0 & 0 & 1 & 0 & 1\end{array}$ & $D_{5}$ & $\boldsymbol{\mu}_{8}$ & 8 & $\begin{array}{lllllll}-11 & 2 & 1 & 1 & 1 & 1 & 1 \\
& & & 1 & & & \end{array}$ & $D_{5}$ \\
\hline $8_{c}$ & $\begin{array}{ccccccc}0 & 1 & 0 & 1 & 0 & 1 & 0 \\
& & & 0 & & & \end{array}$ & $D_{5}$ & $\mu_{8}$ & 8 & $\begin{array}{llllll}-12 & 0 & 1 & 1 & 1 & 1\end{array} 6$ & $D_{5}$ \\
\hline $8_{d}$ & $\begin{array}{ccccccc}1 & 0 & 1 & 0 & 1 & 0 & 1\end{array}$ & $D_{5}$ & $\boldsymbol{\mu}_{8}$ & 8 & $\begin{array}{llllll}-10 & 1 & 1 & 1 & 1 & 1\end{array}$ & $D_{5}$ \\
\hline $8_{e}$ & $\begin{array}{lllllll}1 & 1 & 0 & 0 & 0 & 1 & 1 \\
& & & 1 & & & \end{array}$ & $D_{5}$ & $\boldsymbol{\mu}_{8}$ & 8 & $\begin{array}{lllllll}-8 & 0 & 1 & 1 & 1 & 1 & 2 \\
& & & 1 & & & \end{array}$ & $D_{5}$ \\
\hline $8_{f}$ & $\begin{array}{lllllll}1 & 0 & 0 & 1 & 0 & 0 & 1 \\
& & & 1 & & & \end{array}$ & $D_{5}$ & $\boldsymbol{\mu}_{8}$ & 8 & 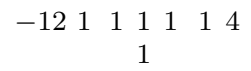 & $D_{5}$ \\
\hline $8_{g}$ & $\begin{array}{ccccccc}0 & 0 & 1 & 0 & 0 & 1 & 1 \\
& & & 1 & & & \end{array}$ & $D_{5}$ & $\boldsymbol{\mu}_{8}$ & 8 & $\begin{array}{lllllll}-6 & 0 & 1 & 1 & 1 & 1 & 0 \\
& & 1 & & & \end{array}$ & $D_{5}$ \\
\hline $8_{h}$ & $\begin{array}{ccccccc}0 & 1 & 0 & 0 & 1 & 1 & 1 \\
& 0 & & & \end{array}$ & $D_{5}$ & $\boldsymbol{\mu}_{8}$ & 8 & 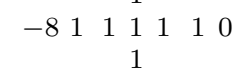 & $D_{5}$ \\
\hline $7_{a}$ & $\begin{array}{lllllll}0 & 1 & 0 & 1 & 0 & 0 & 1\end{array}$ & $A_{6}=E_{7}\left(a_{1}\right)^{2}$ & $\boldsymbol{\mu}_{14}$ & 14 & $\begin{array}{llllll}-10 & 1 & 1 & 1 & 1 & 1\end{array}$ & $E_{7}$ \\
\hline $6_{a}$ & 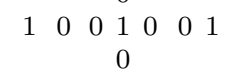 & $E_{7}\left(a_{4}\right)=E_{7}^{3}=-3 A_{2}$ & $G_{26}$ & $6,12,18$ & 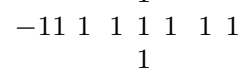 & $E_{7}$ \\
\hline $6_{b}$ & $\begin{array}{ccccccc}0 & 1 & 0 & 0 & 1 & 0 & 1\end{array}$ & $E_{6}\left(a_{2}\right)=E_{6}^{2}$ & $G_{5}$ & 6,12 & 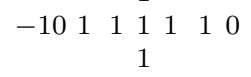 & $E_{6}$ \\
\hline $6_{c}$ & $\begin{array}{llllllll}0 & 1 & 0 & 0 & 0 & 1 & 0 \\
& & & 1 & & & \end{array}$ & $D_{6}\left(a_{2}\right)$ & $G(6,2,2)$ & 6,6 & $\begin{array}{lllllll}-9 & 0 & 1 & 1 & 1 & 1 & 1\end{array}$ & $D_{6}$ \\
\hline $6_{d}$ & $\begin{array}{ccccccc}1 & 0 & 0 & 0 & 0 & 1 & 1 \\
& & & 1 & & & \end{array}$ & $D_{4}$ & $\boldsymbol{\mu}_{6}$ & 6 & $\begin{array}{lllllll}-12 & 0 & 1 & 1 & 1 & 1 & 2\end{array}$ & $D_{4}$ \\
\hline $6{ }_{e}$ & $\begin{array}{ccccccc}0 & 0 & 1 & 0 & 0 & 0 & 1 \\
& & & 1 & & & \end{array}$ & $D_{4}$ & $\boldsymbol{\mu}_{6}$ & 6 & $\begin{array}{llllll}-10 & 0 & 1 & 1 & 1 & 1\end{array}$ & $D_{4}$ \\
\hline $6_{f}$ & $\begin{array}{ccccccc}0 & 0 & 1 & 0 & 0 & 1 & 1 \\
& 0 & & & \end{array}$ & $D_{4}$ & $\mu_{6}$ & 6 & 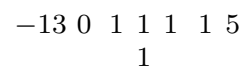 & $D_{4}$ \\
\hline $6_{g}$ & $\begin{array}{lllllll}0 & 0 & 0 & 1 & 0 & 0 & 0 \\
& & & 1 & & & \end{array}$ & $D_{4}$ & $\boldsymbol{\mu}_{6}$ & 6 & 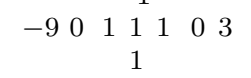 & $D_{4}$ \\
\hline $6_{h}$ & $\begin{array}{ccccccc}0 & 0 & 0 & 0 & 1 & 1 & 1\end{array}$ & $D_{4}$ & $\mu_{6}$ & 6 & $\begin{array}{ccccccc}-6 & 0 & 1 & 1 & 1 & 0 & 0 \\
& 1 & & \end{array}$ & $D_{4}$ \\
\hline $6_{i}$ & 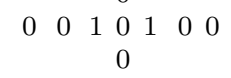 & $A_{5}^{\prime}$ & $\boldsymbol{\mu}_{6}$ & 6 & 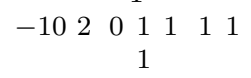 & $A_{5}^{\prime}$ \\
\hline $6_{j}$ & $\begin{array}{llllllll}0 & 0 & 0 & 1 & 0 & 1 & 0\end{array}$ & $A_{5}^{\prime \prime}$ & $\mu_{6}$ & 6 & 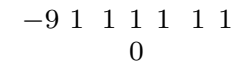 & $A_{5}^{\prime \prime}$ \\
\hline $6_{k}$ & $\begin{array}{lllllll}1 & 1 & 0 & 0 & 0 & 1 & 1 \\
& & & 0 & & & \end{array}$ & $A_{5}^{\prime}$ & $\mu_{6}$ & 6 & $\begin{array}{ccccccc}-6 & 0 & 0 & 1 & 1 & 1 & 1 \\
& & 1 & & & \end{array}$ & $A_{5}^{\prime}$ \\
\hline
\end{tabular}


Table 20 continued: The gradings of positive rank in type $E_{7}$

\begin{tabular}{|c|c|c|c|c|c|c|c|}
\hline No. & Kac diagr & ram & $w$ & $\overline{W(\mathfrak{c}, \theta)}$ & degrees & $\overline{\theta^{\prime}}$ & $L_{\theta}$ \\
\hline $5_{a}$ & $\begin{array}{lllll}0 & 0 & 0 & 1 & 0 \\
& & & 0\end{array}$ & 01 & $A_{4}=D_{6}^{2}$ & $\boldsymbol{\mu}_{10}$ & 10 & $\begin{array}{lllllll}-12 & 1 & 1 & 1 & 1 & 1 & 1 \\
& & & 1 & & & \end{array}$ & $D_{6}$ \\
\hline $5_{b}$ & $\begin{array}{ccccc}0 & 0 & 1 & 0 & 0 \\
& & 0\end{array}$ & 10 & $A_{4}=D_{6}^{2}$ & $\mu_{10}$ & 10 & 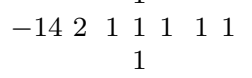 & $D_{6}$ \\
\hline $5_{c}$ & $\begin{array}{ccccc}0 & 1 & 0 & 0 & 0 \\
& & 0\end{array}$ & 11 & $A_{4}=D_{6}^{2}$ & $\mu_{10}$ & 10 & $\begin{array}{lllllll}-10 & 0 & 1 & 1 & 1 & 1 & 1 \\
& & & 1 & & & \end{array}$ & $D_{6}$ \\
\hline $5_{d}$ & $\begin{array}{ccccc}1 & 0 & 0 & 0 & 1 \\
& & & 0\end{array}$ & 01 & $A_{4}$ & $\mu_{5}$ & 5 & $\begin{array}{lllllll}-11 & 1 & 1 & 1 & 1 & 1 & 0 \\
& & 0 & & & \end{array}$ & $A_{4}$ \\
\hline $5_{e}$ & $\begin{array}{lllll}0 & 1 & 0 & 0 & 0 \\
& & & 1\end{array}$ & 01 & $A_{4}$ & $\mu_{5}$ & 5 & $\begin{array}{lllllll}-1 & 1 & 1 & 1 & 1 & 0 & 2 \\
& & & 1 & & \end{array}$ & $A_{4}$ \\
\hline $4_{a}$ & $\begin{array}{ccccc}0 & 0 & 1 & 0 & 0 \\
& 0\end{array}$ & 01 & $D_{4}\left(a_{1}\right)=E_{6}^{3}$ & $G_{8}$ & 8,12 & $\begin{array}{lllllll}-13 & 1 & 1 & 1 & 1 & 1 & 1 \\
& & & 1 & & & \end{array}$ & $E_{6}$ \\
\hline $4_{b}$ & $\begin{array}{ccccc}0 & 0 & 0 & 1 & 0 \\
& & 0\end{array}$ & 00 & $D_{4}\left(a_{1}\right)=E_{6}^{3}$ & $G_{8}$ & 8,12 & 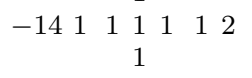 & $E_{6}$ \\
\hline $4_{c}$ & $\begin{array}{ccccc}0 & 0 & 0 & 0 & 1 \\
& & 0\end{array}$ & 01 & $D_{4}\left(a_{1}\right)=E_{6}^{3}$ & $G_{8}$ & 8,12 & $\begin{array}{lllllll}-12 & 1 & 1 & 1 & 1 & 1 & 0 \\
& & 1 & & & \end{array}$ & $E_{6}$ \\
\hline $4_{d}$ & $\begin{array}{ccccc}0 & 1 & 0 & 0 & 0 \\
& & 0 & 0\end{array}$ & 10 & $D_{4}\left(a_{1}\right)$ & $G(4,1,2)$ & 4,8 & $\begin{array}{lllllll}-12 & 0 & 1 & 1 & 1 & 1 & 2 \\
& & & 1 & & & \end{array}$ & $D_{5}$ \\
\hline $4_{e}$ & $\begin{array}{ccccc}1 & 0 & 0 & 0 & 0 \\
& & & 1\end{array}$ & 01 & $D_{4}\left(a_{1}\right)$ & $G(4,1,2)$ & 4,8 & $\begin{array}{lllllll}-12 & 1 & 1 & 1 & 1 & 0 & 2 \\
& & 1 & & \end{array}$ & $D_{5}$ \\
\hline $4_{f}$ & $\begin{array}{ccccc}0 & 0 & 0 & 0 & 0 \\
& & & 1\end{array}$ & 10 & $A_{3}$ & $\boldsymbol{\mu}_{4}$ & 4 & 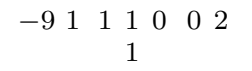 & $A_{4}$ \\
\hline $4_{g}$ & $\begin{array}{ccccc}1 & 0 & 0 & 0 & 0 \\
& & 0\end{array}$ & 11 & $A_{3}$ & $\boldsymbol{\mu}_{4}$ & 4 & $\begin{array}{lllllll}-7 & 1 & 1 & 1 & 0 & 0 & 0 \\
& & 1 & & & \end{array}$ & $A_{4}$ \\
\hline $3_{a}$ & $\begin{array}{ccccc}0 & 0 & 0 & 0 & 1 \\
& 0\end{array}$ & 00 & $3 A_{2}=E_{7}^{6}$ & $G_{26}$ & $6,12,18$ & 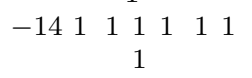 & $E_{7}$ \\
\hline $3_{b}$ & $\begin{array}{ccccc}0 & 1 & 0 & 0 & 0 \\
& & & 0\end{array}$ & 01 & $2 A_{2}$ & $G(6,2,2)$ & 6,6 & $\begin{array}{ccccccc}-12 & 0 & 1 & 1 & 1 & 1 & 1 \\
& 1 & & & \end{array}$ & $D_{6}$ \\
\hline $3_{c}$ & $\begin{array}{ccccc}0 & 0 & 0 & 0 & 0 \\
& & & 1\end{array}$ & 01 & $A_{2}=D_{4}^{2}$ & $\boldsymbol{\mu}_{6}$ & 6 & $\begin{array}{lllllll}-13 & 0 & 1 & 1 & 1 & 1 & 2 \\
& & & 1 & & & \end{array}$ & $D_{4}$ \\
\hline $3 d$ & $\begin{array}{ccccc}0 & 0 & 0 & 0 & 0 \\
& 0\end{array}$ & 11 & $A_{2}=D_{4}^{2}$ & $\boldsymbol{\mu}_{6}$ & 6 & $\begin{array}{lllllll}-9 & 0 & 1 & 1 & 1 & 0 & 0 \\
& & 1 & & & \end{array}$ & $D_{4}$ \\
\hline $2_{a}$ & $\begin{array}{ccccc}0 & 0 & 0 & 0 & 0 \\
& & & 1\end{array}$ & 00 & $7 A_{1}$ & $W\left(E_{7}\right)$ & $2,6,8,10,12,14,18$ & 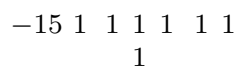 & $E_{7}$ \\
\hline $22_{b}$ & $\begin{array}{ccccc}0 & 0 & 0 & 0 & 0 \\
& 0\end{array}$ & 10 & $4 A_{1}^{\prime \prime}$ & $W\left(F_{4}\right)$ & $2,6,8,12$ & 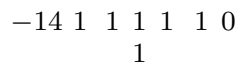 & $E_{6}$ \\
\hline $2_{c}$ & $\begin{array}{ccccc}1 & 0 & 0 & 0 & 0 \\
& 0\end{array}$ & 01 & $3 A_{1}^{\prime}$ & $W\left(B_{3}\right)$ & $2,4,6$ & $\begin{array}{ccccccc}-10 & 0 & 1 & 1 & 1 & 1 & 1\end{array}$ & $A_{5}^{\prime}$ \\
\hline $1_{a}$ & 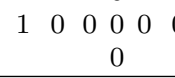 & 00 & $e$ & $W\left(E_{7}\right)$ & $2,6,8,10,12,14,18$ & $\begin{array}{cccccccc}1 & 0 & 0 & 0 & 0 & 0 & 0 \\
& 0 & & & \end{array}$ & $E_{7}$ \\
\hline
\end{tabular}


Table 21: The gradings of positive rank in type $E_{8}$

\begin{tabular}{|c|c|c|c|c|c|c|}
\hline No. & Kac diagram & $w$ & $W(\mathfrak{c}, \theta)$ & degrees & $\theta^{\prime}$ & $L_{\theta}$ \\
\hline $30_{a}$ & $\begin{array}{cccccccc}1 & 1 & 1 & 1 & 1 & 1 & 1 & 1 \\
& & 1 & & & & & \end{array}$ & $E_{8}$ & $\boldsymbol{\mu}_{30}$ & 30 & 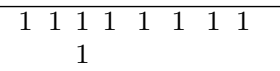 & $E_{8}$ \\
\hline $24_{a}$ & 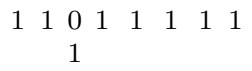 & $E_{8}\left(a_{1}\right)$ & $\boldsymbol{\mu}_{24}$ & 24 & $\begin{array}{lllllll}1 & 1 & 1 & 1 & 1 & 1 & 1-5\end{array}$ & $E_{8}$ \\
\hline $20_{a}$ & $\begin{array}{cccccccc}1 & 1 & 0 & 1 & 0 & 1 & 1 & 1 \\
& 1 & & & & & \end{array}$ & $E_{8}\left(a_{2}\right)$ & $\boldsymbol{\mu}_{20}$ & 20 & $\begin{array}{ccccccc}1 & 1 & 1 & 1 & 1 & 1 & 1-9 \\
& & 1 & & & \end{array}$ & $E_{8}$ \\
\hline $18_{a}$ & 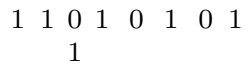 & $E_{7}$ & $\boldsymbol{\mu}_{18}$ & 18 & $\begin{array}{llllllll} & 1 & 1 & 1 & 1 & 1 & 1 & 1-11 \\
& & 1 & & & & \end{array}$ & $E_{7}$ \\
\hline $18_{b}$ & $\begin{array}{cccccccc}1 & 0 & 1 & 0 & 1 & 0 & 1 & 1 \\
& & 1 & & & & & \end{array}$ & $E_{7}$ & $\boldsymbol{\mu}_{18}$ & 18 & 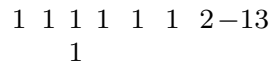 & $E_{7}$ \\
\hline $18_{c}$ & 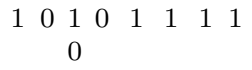 & $E_{7}$ & $\boldsymbol{\mu}_{18}$ & 18 & 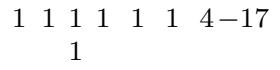 & $E_{7}$ \\
\hline $18_{d}$ & $\begin{array}{cccccccc}0 & 1 & 0 & 1 & 0 & 1 & 1 & 1 \\
& & 1 & & & & & \end{array}$ & $E_{7}$ & $\boldsymbol{\mu}_{18}$ & 18 & $\begin{array}{ccccccc}1 & 1 & 1 & 1 & 1 & 1 & 3\end{array}$ & $E_{7}$ \\
\hline $18_{e}$ & 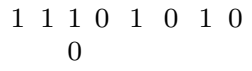 & $E_{7}$ & $\boldsymbol{\mu}_{18}$ & 18 & $\begin{array}{lllllll}1 & 1 & 1 & 1 & 1 & 1 & 0\end{array}$ & $E_{7}$ \\
\hline $15_{a}$ & 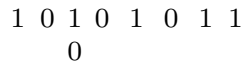 & $E_{8}\left(a_{5}\right)$ & $\boldsymbol{\mu}_{30}$ & 30 & $\begin{array}{ccccccc}1 & 1 & 1 & 1 & 1 & 1 & 1-14 \\
& & 1 & & & & \end{array}$ & $E_{8}$ \\
\hline $14_{a}$ & 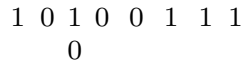 & $E_{7}\left(a_{1}\right)$ & $\boldsymbol{\mu}_{14}$ & 14 & $\begin{array}{lllllll}1 & 1 & 1 & 1 & 1 & 1 & 1-15 \\
& & 1 & & & & \end{array}$ & $E_{7}$ \\
\hline $14_{b}$ & $\begin{array}{cccccccc}1 & 0 & 0 & 1 & 0 & 1 & 0 & 1 \\
& & 1 & & & & & \end{array}$ & $E_{7}\left(a_{1}\right)$ & $\boldsymbol{\mu}_{14}$ & 14 & 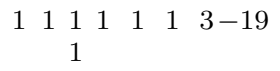 & $E_{7}$ \\
\hline $14_{c}$ & $\begin{array}{cccccccc}0 & 1 & 0 & 0 & 1 & 0 & 1 & 1 \\
& & 1 & & & & & \end{array}$ & $E_{7}\left(a_{1}\right)$ & $\boldsymbol{\mu}_{14}$ & 14 & 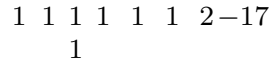 & $E_{7}$ \\
\hline $14_{d}$ & $\begin{array}{llllllll}1 & 0 & 1 & 0 & 1 & 0 & 1 & 0 \\
& 0 & & & & & \end{array}$ & $E_{7}\left(a_{1}\right)$ & $\boldsymbol{\mu}_{14}$ & 14 & $\begin{array}{lllllll}1 & 1 & 1 & 1 & 1 & 1 & 4-21 \\
& & 1 & & & & \end{array}$ & $E_{7}$ \\
\hline $12_{a}$ & $\begin{array}{cccccccc}1 & 0 & 1 & 0 & 0 & 1 & 0 & 1 \\
& 0 & & & & & \end{array}$ & $E_{8}\left(a_{3}\right)$ & $G_{10}$ & 12,24 & $\begin{array}{llllllll} & 1 & 1 & 1 & 1 & 1 & 1 & 1-17 \\
& & 1 & & & & \end{array}$ & $E_{8}$ \\
\hline $12_{b}$ & 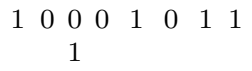 & $E_{6}$ & $\boldsymbol{\mu}_{12}$ & 12 & 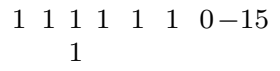 & $E_{6}$ \\
\hline $12_{c}$ & $\begin{array}{cccccccc}0 & 1 & 0 & 0 & 1 & 0 & 0 & 1 \\
& & 1 & & & & & \end{array}$ & $E_{6}$ & $\boldsymbol{\mu}_{12}$ & 12 & $\begin{array}{ccccccc}1 & 1 & 1 & 1 & 1 & 1 & 3\end{array}$ & $E_{6}$ \\
\hline $12_{d}$ & $\begin{array}{cccccccc}0 & 1 & 0 & 1 & 0 & 0 & 1 & 1 \\
& & 0 & & & & & \end{array}$ & $E_{6}$ & $\boldsymbol{\mu}_{12}$ & 12 & 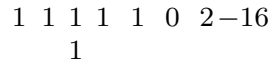 & $E_{6}$ \\
\hline $12_{e}$ & $\begin{array}{cccccccc}0 & 0 & 1 & 0 & 0 & 1 & 1 & 1 \\
& 0 & & & & & \end{array}$ & $E_{6}$ & $\boldsymbol{\mu}_{12}$ & 12 & $\begin{array}{ccccccc}1 & 1 & 1 & 1 & 1 & 0 & 1-14 \\
& & 1 & & & & \end{array}$ & $E_{6}$ \\
\hline $12_{f}$ & 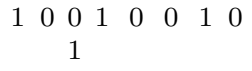 & $E_{6}$ & $\boldsymbol{\mu}_{12}$ & 12 & 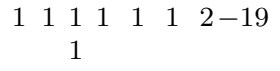 & $E_{6}$ \\
\hline $12_{g}$ & 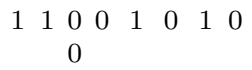 & $E_{6}$ & $\boldsymbol{\mu}_{12}$ & 12 & 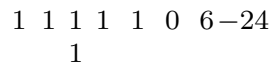 & $E_{6}$ \\
\hline $12_{h}$ & $\begin{array}{cccccccc}0 & 0 & 1 & 0 & 0 & 1 & 0 & 0 \\
& & 1 & & & & & \end{array}$ & $E_{6}$ & $\boldsymbol{\mu}_{12}$ & 12 & $\begin{array}{ccccccc}1 & 1 & 1 & 1 & 1 & 0 & 4-20 \\
& & 1 & & & & \end{array}$ & $E_{6}$ \\
\hline $12_{i}$ & 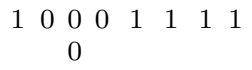 & $E_{6}$ & $\boldsymbol{\mu}_{12}$ & 12 & 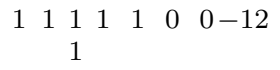 & $E_{6}$ \\
\hline $12_{j}$ & 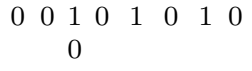 & $D_{7}$ & $\boldsymbol{\mu}_{12}$ & 12 & $\begin{array}{llllllll}0 & 1 & 1 & 1 & 1 & 1 & 1-15 \\
& & 1 & & & & & \end{array}$ & $D_{7}$ \\
\hline $10_{a}$ & $\begin{array}{llllllll}0 & 0 & 1 & 0 & 0 & 1 & 0 & 1 \\
& & 0 & & & & & \end{array}$ & $E_{8}\left(a_{6}\right)=-2 A_{4}$ & $G_{16}$ & 20,30 & 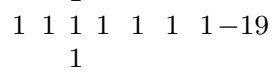 & $E_{8}$ \\
\hline $10_{b}$ & $\begin{array}{cccccccc}1 & 0 & 0 & 0 & 1 & 0 & 0 & 1 \\
& 1 & & & & & \end{array}$ & $D_{6}$ & $\boldsymbol{\mu}_{10}$ & 10 & 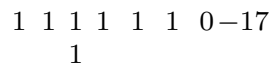 & $D_{6}$ \\
\hline $10_{c}$ & 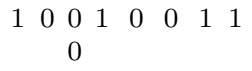 & $D_{6}$ & $\boldsymbol{\mu}_{10}$ & 10 & $\begin{array}{ccccccc}2 & 1 & 1 & 1 & 1 & 1 & 1-21 \\
& & 1 & & & & \end{array}$ & $D_{6}$ \\
\hline $10_{d}$ & $\begin{array}{cccccccc}1 & 0 & 1 & 0 & 0 & 0 & 1 & 0 \\
& 0 & & & & & \end{array}$ & $D_{6}$ & $\boldsymbol{\mu}_{10}$ & 10 & $\begin{array}{llllllll}0 & 1 & 1 & 1 & 1 & 1 & 1-17 \\
& & 1 & & & & & \end{array}$ & $D_{6}$ \\
\hline $10_{e}$ & $\begin{array}{llllllll}0 & 1 & 0 & 0 & 1 & 0 & 1 & 0 \\
& & 0 & & & & & \end{array}$ & $D_{6}$ & $\boldsymbol{\mu}_{10}$ & 10 & 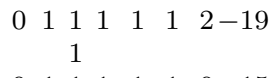 & $D_{6}$ \\
\hline $10_{f}$ & 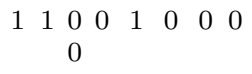 & $D_{6}$ & $\boldsymbol{\mu}_{10}$ & 10 & $\begin{array}{lllllll}0 & 1 & 1 & 1 & 1 & 1 & 0\end{array}$ & $D_{6}$ \\
\hline
\end{tabular}


Table 21 continued: The gradings of positive rank in type $E_{8}$

\begin{tabular}{|c|c|c|c|c|c|c|}
\hline No. & Kac diagram & $w$ & $W(\mathfrak{c}, \theta)$ & degrees & $\theta^{\prime}$ & $L_{\theta}$ \\
\hline $9_{a}$ & $\begin{array}{llllllll}0 & 0 & 1 & 0 & 0 & 0 & 1 & 1 \\
& & 0 & & & & & \end{array}$ & $E_{6}\left(a_{1}\right)=E_{7}^{2}$ & $\boldsymbol{\mu}_{18}$ & 18 & $\begin{array}{llllllll} & 1 & 1 & 1 & 1 & 1 & 1 & 1-20 \\
& & 1 & & & & \end{array}$ & $E_{7}$ \\
\hline $9_{b}$ & 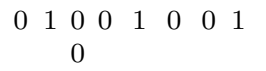 & $E_{6}\left(a_{1}\right)=E_{7}^{2}$ & $\boldsymbol{\mu}_{18}$ & 18 & 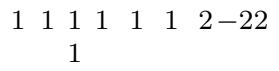 & $E_{7}$ \\
\hline $9_{c}$ & $\begin{array}{cccccccc}1 & 0 & 0 & 1 & 0 & 0 & 1 & 0 \\
& 0 & & & & & \end{array}$ & $E_{6}\left(a_{1}\right)=E_{7}^{2}$ & $\boldsymbol{\mu}_{18}$ & 18 & 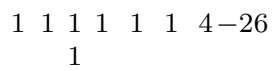 & $E_{7}$ \\
\hline $9_{d}$ & $\begin{array}{cccccccc}0 & 0 & 1 & 0 & 0 & 1 & 0 & 0 \\
& 0 & & & & & \end{array}$ & $E_{6}\left(a_{1}\right)=E_{7}^{2}$ & $\boldsymbol{\mu}_{18}$ & 18 & 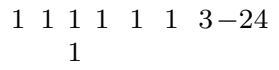 & $E_{7}$ \\
\hline $9_{e}$ & 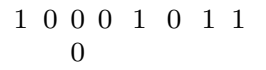 & $E_{6}\left(a_{1}\right)=E_{7}^{2}$ & $\boldsymbol{\mu}_{18}$ & 18 & 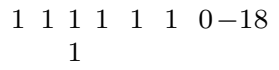 & $E_{7}$ \\
\hline $9_{f}$ & 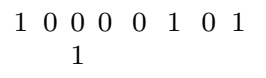 & $E_{6}\left(a_{1}\right)$ & $\boldsymbol{\mu}_{9}$ & 9 & 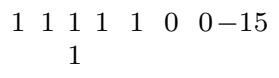 & $E_{6}$ \\
\hline $8_{a}$ & $\begin{array}{llllllll}0 & 0 & 1 & 0 & 0 & 0 & 1 & 0 \\
& 0 & & & & & \end{array}$ & $D_{8}\left(a_{3}\right)$ & $G_{9}$ & 8,24 & 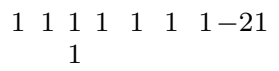 & $E_{8}$ \\
\hline $8_{b}$ & 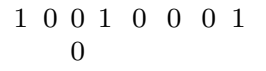 & $D_{5}$ & $\boldsymbol{\mu}_{8}$ & 8 & $\begin{array}{llllllll}1 & 1 & 1 & 1 & 1 & 1 & 2 & -23 \\
& & 1 & & & & & \end{array}$ & $D_{5}$ \\
\hline $8_{c}$ & $\begin{array}{cccccccc}0 & 1 & 0 & 0 & 0 & 1 & 0 & 1 \\
& & 0 & & & & & \end{array}$ & $D_{5}$ & $\boldsymbol{\mu}_{8}$ & 8 & 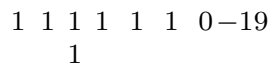 & $D_{5}$ \\
\hline $8_{d}$ & 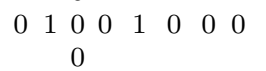 & $D_{5}$ & $\boldsymbol{\mu}_{8}$ & 8 & 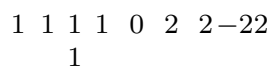 & $D_{5}$ \\
\hline $8_{e}$ & $\begin{array}{cccccccc}0 & 0 & 0 & 0 & 1 & 0 & 0 & 1 \\
& 1 & & & & & \end{array}$ & $D_{5}$ & $\mu_{8}$ & 8 & 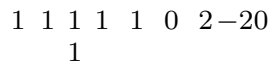 & $D_{5}$ \\
\hline $8_{f}$ & $\begin{array}{cccccccc}0 & 0 & 0 & 1 & 0 & 0 & 1 & 1 \\
& 0 & & & & & \end{array}$ & $D_{5}$ & $\mu_{8}$ & 8 & 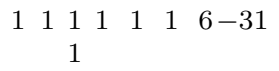 & $D_{5}$ \\
\hline $8_{g}$ & 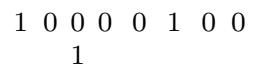 & $D_{5}$ & $\boldsymbol{\mu}_{8}$ & 8 & 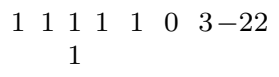 & $D_{5}$ \\
\hline $8_{h}$ & $\begin{array}{cccccccc}1 & 0 & 0 & 0 & 1 & 0 & 1 & 0 \\
& 0 & & & & & \end{array}$ & $D_{5}$ & $\boldsymbol{\mu}_{8}$ & 8 & 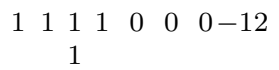 & $D_{5}$ \\
\hline $8_{i}$ & 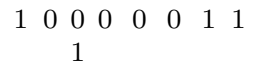 & $D_{5}$ & $\boldsymbol{\mu}_{8}$ & 8 & 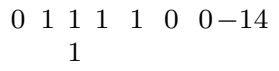 & $D_{5}$ \\
\hline $8_{j}$ & 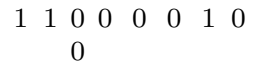 & $D_{5}$ & $\boldsymbol{\mu}_{8}$ & 8 & $\begin{array}{lllllll}1 & 1 & 1 & 1 & 1 & 0 & 4\end{array}$ & $D_{5}$ \\
\hline $8_{k}$ & 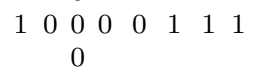 & $D_{5}$ & $\mu_{8}$ & 8 & 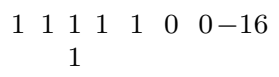 & $D_{5}$ \\
\hline $7_{a}$ & $\begin{array}{cccccccc}0 & 0 & 0 & 1 & 0 & 0 & 1 & 0 \\
& 0 & & & & & \end{array}$ & $A_{6}=E_{7}\left(a_{1}\right)^{2}$ & $\boldsymbol{\mu}_{14}$ & 14 & $\begin{array}{ccccccc}1 & 1 & 1 & 1 & 1 & 1 & 1-22 \\
& & 1 & & & & \end{array}$ & $E_{7}$ \\
\hline $7_{b}$ & 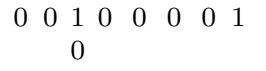 & $A_{6}=E_{7}\left(a_{1}\right)^{2}$ & $\boldsymbol{\mu}_{14}$ & 14 & 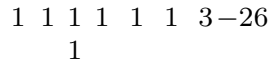 & $E_{7}$ \\
\hline $7_{c}$ & 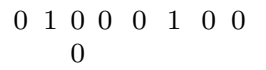 & $A_{6}=E_{7}\left(a_{1}\right)^{2}$ & $\boldsymbol{\mu}_{14}$ & 14 & 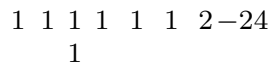 & $E_{7}$ \\
\hline $7_{d}$ & $\begin{array}{cccccccc}1 & 0 & 0 & 0 & 1 & 0 & 0 & 1 \\
& 0 & & & & & \end{array}$ & $A_{6}=E_{7}\left(a_{1}\right)^{2}$ & $\boldsymbol{\mu}_{14}$ & 14 & 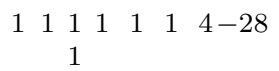 & $E_{7}$ \\
\hline $6_{a}$ & $\begin{array}{cccccccc}0 & 0 & 0 & 1 & 0 & 0 & 0 & 1 \\
& 0 & & & & & \end{array}$ & $E_{8}\left(a_{8}\right)=-4 A_{2}$ & $G_{32}$ & $12,18,24,30$ & $\begin{array}{llllllll} & 1 & 1 & 1 & 1 & 1 & 1 & 1-23 \\
& & 1 & & & & \end{array}$ & $E_{8}$ \\
\hline $6_{b}$ & $\begin{array}{cccccccc}0 & 1 & 0 & 0 & 0 & 0 & 1 & 0 \\
& 0 & & & & \end{array}$ & $E_{7}\left(a_{4}\right)=E_{7}^{3}$ & $G_{26}$ & $6,12,18$ & 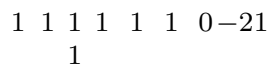 & $E_{7}$ \\
\hline $6_{c}$ & 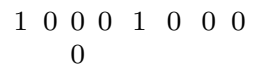 & $D_{6}\left(a_{2}\right)$ & $G(6,1,2)$ & 6,12 & $\begin{array}{lllllll}0 & 1 & 1 & 1 & 1 & 1 & 1-21 \\
& & 1 & & & & \end{array}$ & $D_{7}$ \\
\hline $6_{d}$ & $\begin{array}{cccccccc}0 & 0 & 0 & 0 & 0 & 1 & 0 & 0 \\
& & 1 & & & & & \end{array}$ & $E_{6}\left(a_{2}\right)$ & $G_{5}$ & 6,12 & 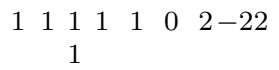 & $E_{6}$ \\
\hline $6_{e}$ & 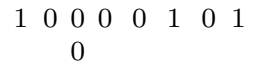 & $E_{6}\left(a_{2}\right)$ & $G_{5}$ & 6,12 & 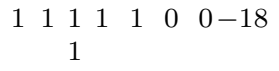 & $E_{6}$ \\
\hline $6_{f}$ & 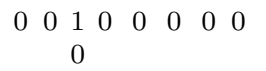 & $A_{5}$ & $\boldsymbol{\mu}_{6}$ & 6 & 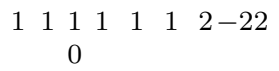 & $A_{5}$ \\
\hline $6 g$ & $\begin{array}{cccccccc}0 & 0 & 0 & 0 & 1 & 0 & 1 & 0 \\
& 0 & & & & & \end{array}$ & $A_{5}$ & $\boldsymbol{\mu}_{6}$ & 6 & 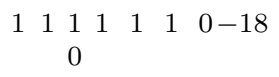 & $A_{5}$ \\
\hline $6_{h}$ & 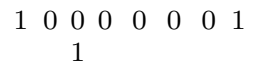 & $D_{4}$ & $\mu_{6}$ & 6 & 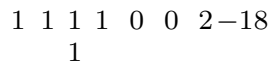 & $D_{4}$ \\
\hline $6_{i}$ & $\begin{array}{cccccccc}0 & 0 & 0 & 0 & 0 & 0 & 1 & 1 \\
& & 1 & & & & & \end{array}$ & $D_{4}$ & $\boldsymbol{\mu}_{6}$ & 6 & 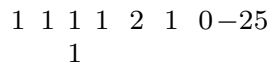 & $D_{4}$ \\
\hline $6_{j}$ & $\begin{array}{cccccccc}0 & 0 & 0 & 0 & 0 & 1 & 1 & 1 \\
& 0 & & & & & \end{array}$ & $D_{4}$ & $\boldsymbol{\mu}_{6}$ & 6 & 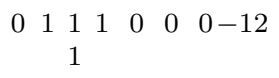 & $D_{4}$ \\
\hline $6_{k}$ & 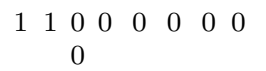 & $D_{4}$ & $\boldsymbol{\mu}_{6}$ & 6 & $\begin{array}{lllllll}0 & 1 & 1 & 1 & 0 & 0 & 3-18 \\
& & 1 & & & & \end{array}$ & $D_{4}$ \\
\hline
\end{tabular}


Table 21 continued: The gradings of positive rank in type $E_{8}$

\begin{tabular}{|c|c|c|c|c|c|c|}
\hline No. & Kac diagram & $w$ & $W(\mathfrak{c}, \theta)$ & degrees & $\theta^{\prime}$ & $L_{\theta}$ \\
\hline $5_{a}$ & 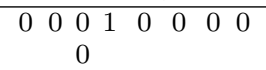 & $2 A_{4}=E_{8}^{6}$ & $G_{16}$ & 20,30 & $\begin{array}{llllllll} & 1 & 1 & 1 & 1 & 1 & 1-24 \\
& & 1 & & & & & \end{array}$ & $E_{8}$ \\
\hline $5_{b}$ & $\begin{array}{cccccccc}0 & 1 & 0 & 0 & 0 & 0 & 0 & 1 \\
& 0 & & & & \end{array}$ & $A_{4}$ & $\boldsymbol{\mu}_{10}$ & 10 & $\begin{array}{ccccccc}1 & 1 & 1 & 1 & 1 & 1 & 0-22 \\
& 1 & & & & \end{array}$ & $D_{6}$ \\
\hline $5_{c}$ & $\begin{array}{llllllll}0 & 0 & 0 & 0 & 0 & 0 & 1 & 0 \\
& & 1 & & & & & \end{array}$ & $A_{4}$ & $\boldsymbol{\mu}_{10}$ & 10 & $\begin{array}{ccccccc}2 & 1 & 1 & 1 & 1 & 1 & 1-26 \\
& & 1 & & & & \end{array}$ & $D_{6}$ \\
\hline $5_{d}$ & $\begin{array}{llllllll}0 & 0 & 0 & 0 & 1 & 0 & 0 & 1 \\
& & 0 & & & & & \end{array}$ & $A_{4}$ & $\boldsymbol{\mu}_{10}$ & 10 & $\begin{array}{ccccccc}1 & 1 & 1 & 1 & 1 & 1 & 4\end{array}$ & $D_{6}$ \\
\hline $5_{e}$ & 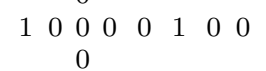 & $A_{4}$ & $\boldsymbol{\mu}_{10}$ & 10 & $\begin{array}{ccccccc}0 & 1 & 1 & 1 & 1 & 1 & 2-24 \\
& 1 & & & & & \end{array}$ & $D_{6}$ \\
\hline $5_{f}$ & $\begin{array}{cccccccc}1 & 0 & 0 & 0 & 0 & 0 & 1 & 1 \\
& 0 & & & & & \end{array}$ & $A_{4}$ & $\boldsymbol{\mu}_{10}$ & 10 & $\begin{array}{cccccccc}0 & 1 & 1 & 1 & 1 & 1 & 0 & -20 \\
& & 1 & & & & & \end{array}$ & $D_{6}$ \\
\hline $4_{a}$ & $\begin{array}{cccccccc}0 & 0 & 0 & 0 & 1 & 0 & 0 & 0 \\
& 0 & & & & & \end{array}$ & $2 D_{4}\left(a_{1}\right)$ & $G_{31}$ & $8,12,20,24$ & $\begin{array}{ccccccc}1 & 1 & 1 & 1 & 1 & 1 & 1-25 \\
& & 1 & & & & \end{array}$ & $E_{8}$ \\
\hline $4_{b}$ & $\begin{array}{llllllll}0 & 0 & 0 & 0 & 0 & 0 & 0 & 1 \\
& & 1 & & & & & \end{array}$ & $D_{4}\left(a_{1}\right)=E_{6}^{3}$ & $G_{8}$ & 8,12 & 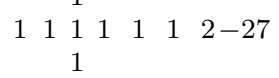 & $E_{6}$ \\
\hline $4_{c}$ & $\begin{array}{llllllll}0 & 1 & 0 & 0 & 0 & 0 & 0 & 0 \\
& 0 & & & & \end{array}$ & $D_{4}\left(a_{1}\right)=E_{6}^{3}$ & $G_{8}$ & 8,12 & $\begin{array}{lllllll}1 & 1 & 1 & 1 & 1 & 0 & 2-24 \\
& 1 & & & & \end{array}$ & $E_{6}$ \\
\hline $4_{d}$ & $\begin{array}{cccccccc}0 & 0 & 0 & 0 & 0 & 1 & 0 & 1 \\
& 0 & & & & & \end{array}$ & $D_{4}\left(a_{1}\right)=E_{6}^{3}$ & $G_{8}$ & 8,12 & $\begin{array}{ccccccc}1 & 1 & 1 & 1 & 1 & 0 & 0-20 \\
& 1 & & & & \end{array}$ & $E_{6}$ \\
\hline $4_{e}$ & 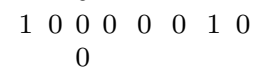 & $D_{4}\left(a_{1}\right)=D_{5}^{2}$ & $G(4,1,2)$ & 4,8 & $\begin{array}{ccccccc}1 & 1 & 1 & 1 & 0 & 0 & 0 \\
& 1 & & & & & \end{array}$ & $D_{5}$ \\
\hline $3_{a}$ & $\begin{array}{cccccccc}0 & 0 & 0 & 0 & 0 & 0 & 0 & 0 \\
& 1 & & & & \end{array}$ & $4 A_{2}=E_{8}^{10}$ & $G_{32}$ & $12,18,24,30$ & $\begin{array}{ccccccc}1 & 1 & 1 & 1 & 1 & 1 & 1-26 \\
& 1 & & & \end{array}$ & $E_{8}$ \\
\hline $3_{b}$ & $\begin{array}{cccccccc}0 & 0 & 0 & 0 & 0 & 1 & 0 & 0 \\
& 0 & & & & & \end{array}$ & $3 A_{2}=E_{7}^{6}$ & $G_{26}$ & $6,12,18$ & $\begin{array}{ccccccc}1 & 1 & 1 & 1 & 1 & 1 & 0-24 \\
& & & & & & \end{array}$ & $E_{7}$ \\
\hline $3_{c}$ & 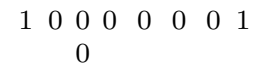 & $2 A_{2}=D_{7}^{4}$ & $G(6,1,2)$ & 6,12 & $\begin{array}{cccccccc}0 & 1 & 1 & 1 & 1 & 1 & 1-24 \\
& & 1 & & & & & \end{array}$ & $D_{7}$ \\
\hline $3_{d}$ & $\begin{array}{cccccccc}0 & 0 & 0 & 0 & 0 & 0 & 1 & 1 \\
& 0 & & & & & \end{array}$ & $A_{2}=D_{4}^{2}$ & $\boldsymbol{\mu}_{6}$ & 6 & 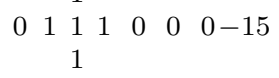 & $D_{4}$ \\
\hline $2_{a}$ & 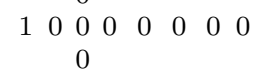 & $8 A_{1}=-1$ & $W\left(E_{8}\right)$ & $2,8,12,14,18,20,24,30$ & $\begin{array}{lllllll}1 & 1 & 1 & 1 & 1 & 1 & 1-27\end{array}$ & $E_{8}$ \\
\hline $2_{b}$ & 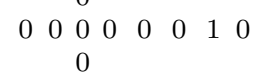 & $4 A_{1}^{\prime \prime}=E_{6}^{6}$ & $W\left(F_{4}\right)$ & $2,6,8,12$ & $\begin{array}{ccccccc}1 & 1 & 1 & 1 & 1 & 0 & 0 \\
& 1 & & & & -22\end{array}$ & $E_{6}$ \\
\hline $1_{a}$ & $\begin{array}{cccccccc}0 & 0 & 0 & 0 & 0 & 0 & 0 & 1 \\
& 0 & & & & & \end{array}$ & 1 & $W\left(E_{8}\right)$ & $2,8,12,14,18,20,24,30$ & $\begin{array}{cccccccc}0 & 0 & 0 & 0 & 0 & 0 & 0 & 1 \\
& 0 & & & & & \end{array}$ & $E_{8}$ \\
\hline
\end{tabular}

\section{Little Weyl groups for inner type $E$ and Kostant sections}

In this section we compute the little Weyl groups $W(\mathfrak{c}, \theta)$ and their degrees when $\theta$ is inner of positive rank in type $E$. As a byproduct we show that every positive rank inner automorphism is principal in a Levi subgroup. This leads to a verification of Popov's conjecture on the existence of Kostant sections, and gives a characterization of the orders of positive-rank automorphisms.

\subsection{The Levi subgroup $L_{\theta}$}

In tables 19-21 above we have indicated a Levi subgroup $L_{\theta}$ whose corresponding subset $J \subset\{1, \ldots, \ell\}$ satisfies the conditions of Lemma 7.6, giving an embedding

$$
C_{W_{J}}(w) \hookrightarrow W(\mathfrak{c}, \theta) .
$$

In each case, the embedding (14) turns out to be an isomorphism. It follows that the degrees of $W(\mathfrak{c}, \theta)$ are those degrees of $W_{J}$ which are divisible by $m$. 
We verify that (14) is an isomorphism as follows. Let $U_{J} \subset W$ be the subgroup acting trivially on the span of the roots $\alpha_{j}$ for $j \in J$ and set $c_{J}(w)=\left|C_{W}(w)\right| /\left|U_{J}\right|$. Lemma 7.5 shows that $|W(\mathfrak{c}, \theta)|$ divides $c_{J}(w)$. The subgroup $U_{J}$ can be found in the tables of [7] (it is denoted there by $W_{2}$ ). In all but eight cases we find that

$$
\left|C_{W_{J}}(w)\right|=c_{J}(w)
$$

showing that $C_{W_{J}}(w)=W(\mathfrak{c}, \theta)$.

We list the exceptional cases for which $\left|C_{W_{J}}(w)\right|<c_{J}(w)$. We write $\left|C_{W_{J}}(w)\right|$ as the product of degrees divisible by $m$.

\begin{tabular}{cccccc}
\hline$G$ & no. & $w$ & $J$ & $\left|C_{W_{J}}(w)\right|$ & $c_{J}(w)$ \\
\hline$E_{6}$ & $4_{b}$ & $D_{4}\left(a_{1}\right)$ & $D_{5}$ & $4 \cdot 8$ & $8 \cdot 12$ \\
$E_{7}$ & $9_{b}$ & $E_{6}\left(a_{1}\right)$ & $E_{6}$ & 9 & 18 \\
$E_{7}$ & $5_{d}, 5_{e}$ & $A_{4}$ & $A_{4}$ & 5 & 10 \\
$E_{7}$ & $4_{d}, 4_{e}$ & $D_{4}\left(a_{1}\right)$ & $D_{5}$ & $4 \cdot 8$ & $8 \cdot 12$ \\
$E_{8}$ & $9_{f}$ & $E_{6}\left(a_{1}\right)$ & $E_{6}$ & 9 & 18 \\
$E_{8}$ & $4_{e}$ & $D_{4}\left(a_{1}\right)$ & $D_{5}$ & $4 \cdot 8$ & $8 \cdot 12$ \\
\hline
\end{tabular}

To show that $W(\mathfrak{c}, \theta)=C_{W_{J}}(w)$ in all of these cases as well, it suffices to show that $G_{0}$ has an invariant polynomial of degree $d=4,9,5,4,9,4$ for the respective rows. If $k$ has characteristic zero this can be done using the computer algebra system $\mathrm{LiE}$ to find the dimension of the $G_{0}$-invariants in $\operatorname{Sym}^{d}\left(\mathfrak{g}_{1}^{*}\right)$. In fact we did this for all of the positive rank cases in exceptional groups, as a confirmation of our tables. If $k$ has positive characteristic $p$ (not dividing $m$ ) the desired invariant is provided by the following result which is apparently standard, but we could not find a reference.

Lemma 10.1 Let $\rho: H \rightarrow \mathrm{GL}(V)$ be a rational representation of a reductive algebraic group $H$ over the ring $\mathbb{Z}[\zeta]$, where $\zeta \in \overline{\mathbb{Q}}$ is a primitive $m^{\text {th }}$-root of unity. Assume that $H(\overline{\mathbb{Q}})$ has a nonzero invariant vector in $V(\overline{\mathbb{Q}})$ with multiplicity one. Then $H(k)$ has a nonzero invariant in $V(k)$ for any algebraically-closed field $k$ of characteristic $p$ not dividing $m$.

Proof: Let $W(k)$ be the ring of Witt vectors of $k$, let $K$ be the quotient field of $W(k)$ and let $L$ be an algebraic closure of $K$. Our assumption implies, via complete reducibility, that $\operatorname{dim}_{L} V(L)^{H(L)}=$ $\operatorname{dim}_{\overline{\mathbb{Q}}} V(\overline{\mathbb{Q}})^{H(\overline{\mathbb{Q}})}=1$. Let $f \in V(L)$ be a generator of $V(L)^{H(L)}$. The line $L \cdot f$ is preserved by $\operatorname{Gal}(L / K)$, so Hilbert's theorem 90 implies that $L \cdot f \cap V(K)$ is nonzero. We may therefore assume that $f \in V(K)$. Clearing denominators, we may further assume that $f \in V(W(k))$ and is nonzero modulo the maximal ideal $M$ of $W(k)$. The reduction of $f$ modulo $M$ gives a nonzero invariant of $H(k)$ in $V(k)$.

As illustrated in the following examples, we can often compute the desired invariant by hand. 


\subsubsection{Example: $E_{6}$ no. $4_{b}$}

We label the affine diagram of $E_{6}$ and write the Kac diagram of $\theta$ respectively as as shown:

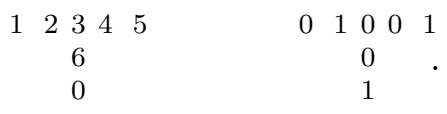

We view $\mathfrak{g}_{1}$ as a representation of $\mathrm{SL}_{2} \times \mathrm{SL}_{4} \times T_{2}$, where $T_{2}$ is the two dimensional torus whose cocharacter group has basis $\left\{\breve{\omega}_{2}, \breve{\omega}_{5}\right\}$, where $\check{\omega}_{i}$ are the fundamental co-weights of $E_{6}$. Each node $i$ labelled 1 in the Kac diagram gives a summand $V_{i}$ of $\mathfrak{g}_{1}^{*}$ whose highest weight is the fundamental weight on each node adjacent to $i$ and with central character $\alpha_{i}$ restricted to $T_{2}$. Thus, we have

$$
\begin{aligned}
& \mathfrak{g}_{1}^{*} \simeq(2 \otimes 6) \quad(\quad(1 \otimes \check{4}) \oplus \quad(1 \otimes 4) \\
& \check{\omega}_{2}=\begin{array}{lll}
1 & -2 & 0
\end{array} \\
& \check{\omega}_{5}=\begin{array}{lll}
0 & -1 & 1
\end{array}
\end{aligned}
$$

Here 2 and 4 are the standard representations of $\mathrm{SL}_{2}$ and $\mathrm{SL}_{4}, \check{4}$ is the dual of 4 and $6=\Lambda^{2} \mathbf{4}$. It follows that the symmetric algebra of $\mathfrak{g}_{1}^{*}$ can have $G_{0}$-invariants only in tri-degrees $(2 k, k, k)$. To find the expected invariant of degree four, we must find an $\mathrm{SL}_{2} \times \mathrm{SL}_{4}$-invariant in the summand for $k=1$ :

$$
\operatorname{Sym}^{2}(\mathbf{2} \otimes \mathbf{6}) \otimes(\mathbf{1} \otimes \check{4}) \otimes(\mathbf{1} \otimes \mathbf{4})=\operatorname{Sym}^{2}(\mathbf{2} \otimes \mathbf{6}) \otimes(\mathbf{1} \otimes \operatorname{End}(\mathbf{4})) .
$$

Since $m=4$ we have $p \neq 2$, so $\operatorname{End}(4)=\mathbf{1} \oplus \mathfrak{s l}_{4}$. Since 2 and $\mathbf{6}$ are self-dual, affording alternating and symmetric forms, respectively, our invariant must be given by an $\mathrm{SL}_{2} \times \mathrm{SL}_{4}$-equivariant mapping $\operatorname{Sym}^{2}(\mathbf{2} \otimes \mathbf{6}) \rightarrow \mathbf{1} \otimes \mathfrak{s l}_{4}$. Indeed, wedging in both factors gives a map

$$
\operatorname{Sym}^{2}(2 \otimes 6) \longrightarrow \Lambda^{2} \mathbf{2} \otimes \Lambda^{2} \mathbf{6}=1 \otimes \mathfrak{s o}_{6} \simeq 1 \otimes \mathfrak{s l}_{4},
$$

exhibiting the desired invariant of degree four.

\subsubsection{Example: $E_{7}$ no. $5_{d}$}

The Kac diagram is

$$
\begin{array}{llllllll}
0 & 1 & 0 & 0 & 0 & 0 & 1
\end{array}
$$

with $G_{0}^{s c}=\mathrm{SL}_{2} \times \mathrm{SL}_{5}$ and

$$
\mathfrak{g}_{1}^{*}=(2 \otimes 5) \oplus(1 \otimes \check{5}) \oplus\left(1 \otimes \Lambda^{2} 5\right) .
$$

The center of $G_{0}$ has invariants in tri-degrees $(2 k, k, 2 k)$, leading us to seek an $\mathrm{SL}_{5}$-equivariant mapping

$$
\mathbf{5} \otimes \operatorname{Sym}^{2}\left(\Lambda^{2} \check{\mathbf{5}}\right) \longrightarrow \operatorname{Sym}^{2}(2 \otimes \mathbf{5})^{\mathrm{SL}_{2}} .
$$

Let $U$ and $V$ be $k$-vector spaces of dimensions 2 and arbitrary $n<\infty$, respectively. Let $P_{2}(\operatorname{Hom}(V, U))$ be the space of degree two-polynomials on $\operatorname{Hom}(V, U)$, with the natural $S L(V) \times \mathrm{SL}(U)$-action. Then we have a nonzero (hence injective) mapping

$$
\varphi: \Lambda^{2}(V) \longrightarrow P_{2}(\operatorname{Hom}(V, U))^{\mathrm{SL}(U)}, \quad \omega \mapsto \varphi_{\omega}
$$


given by $\varphi_{\omega}(f)=f_{*}(\omega)$, where $f_{*}: \Lambda^{2}(V) \rightarrow \Lambda^{2}(U) \simeq k$ is the map induced by $f$. One checks that $\operatorname{dim} P_{2}(\operatorname{Hom}(V, U))^{\operatorname{SL}(U)}=\left(\begin{array}{l}n \\ 2\end{array}\right)$, so that $\varphi$ is an isomorphism of $\operatorname{SL}(V)$-modules

$$
\Lambda^{2}(V) \simeq P_{2}(\operatorname{Hom}(V, U))^{\mathrm{SL}(U)} .
$$

Returning to our task, we now must find an $\mathrm{SL}_{5}$-equivariant mapping

$$
\mathbf{5} \otimes \operatorname{Sym}^{2}\left(\Lambda^{2} \check{5}\right) \longrightarrow \Lambda^{2} \mathbf{5} .
$$

The contraction mapping

$$
\mathbf{5} \otimes \Lambda^{2} \check{\mathbf{5}} \longrightarrow \check{\mathbf{5}}, \quad v \otimes \omega \mapsto c_{v}(\omega),
$$

where $c_{v}(\lambda \wedge \mu)=\langle\lambda, v\rangle \mu-\langle\mu, v) \lambda$, extends to a mapping

$$
\mathbf{5} \otimes \operatorname{Sym}^{2}\left(\Lambda^{2} \check{\mathbf{5}}\right) \longrightarrow \Lambda^{3} \check{\mathbf{5}}, \quad v \otimes(\omega \cdot \eta) \mapsto c_{v}(\omega) \wedge \eta+c_{v}(\eta) \wedge \omega .
$$

Since $\Lambda^{3} \check{\mathbf{5}} \simeq \Lambda^{2} \mathbf{5}$ as $\mathrm{SL}_{5}$-modules, we have the desired invariant.

\subsubsection{Example: $E_{7}$ no. $4_{d}$}

The Kac diagram is

$$
\begin{array}{lllllll}
1 & 0 & 0 & 0 & 0 & 0 & 1 \\
& & & 1 & &
\end{array}
$$

and $G_{0}^{s c}=\mathrm{SL}_{6}$ with $\mathfrak{g}_{1}=\mathbf{6} \oplus \check{\mathbf{6}} \oplus \Lambda^{3} \mathbf{6}$. The action of the center leads us to seek an $\mathrm{SL}_{6}$-invariant in

$$
\mathbf{6} \otimes \check{\mathbf{6}} \otimes \operatorname{Sym}^{2}\left(\Lambda^{3} \mathbf{6}\right)
$$

If $V$ is a $k$-vector space of even dimension $2 m$, we have a nonzero $\mathrm{SL}(V)$-equivariant mapping

$$
\varphi: \operatorname{End}(V) \longrightarrow P_{2}\left(\Lambda^{m} V\right), \quad A \mapsto \varphi_{A},
$$

given by $\varphi_{A}(\omega)=\omega \wedge A_{*} \omega$. Since the $\operatorname{SL}(V)$-module $\Lambda^{m} V$ is self-dual this may be viewed as a nonzero mapping $\operatorname{End}(V) \rightarrow \operatorname{Sym}^{2}\left(\Lambda^{m} V\right)$. Taking $m=3$ gives the desired invariant.

\subsubsection{Example: $E_{7}$ no. $4_{e}$}

The Kac diagram is

$$
\begin{array}{lllllll}
0 & 1 & 0 & 0 & 0 & 1 & 0
\end{array}
$$

with $G_{0}^{s c}=H_{1} \times \operatorname{Spin}_{8} \times H_{2}$, where $H_{1} \simeq H_{2} \simeq \mathrm{SL}_{2}$, and $\mathfrak{g}_{1}^{*}=(\mathbf{2} \otimes \mathbf{8} \otimes \mathbf{1}) \oplus\left(\mathbf{1} \otimes \mathbf{8}^{\prime} \otimes \mathbf{2}\right)$, where 8 and $\mathbf{8}^{\prime}$ are non-isomorphic eight dimensional irreducible representations of $\operatorname{Spin}_{8}$. The action of the center leads us to seek an invariant in

$$
\operatorname{Sym}^{2}(2 \otimes 8 \otimes 1) \otimes \operatorname{Sym}^{2}\left(1 \otimes 8^{\prime} \otimes 2\right) .
$$

Since every representation in sight is self-dual, we require a $\operatorname{Spin}_{8}$-equivariant mapping from the $H_{1^{-}}$ coinvariants to the $\mathrm{H}_{2}$-invariants:

$$
\operatorname{Sym}^{2}(2 \otimes 8 \otimes 1)_{H_{1}} \longrightarrow \operatorname{Sym}^{2}\left(1 \otimes 8^{\prime} \otimes 2\right)^{H_{2}} .
$$


Since $m=4$, the characteristic of $k$ is not two, so for a $k$-vector space $V$ of arbitrary finite dimension $n$ the symmetric square

$$
\operatorname{Sym}^{2}(V \otimes \mathbf{2})=\operatorname{Sym}^{2}\left(\mathbf{2}^{\oplus n}\right)=n \cdot \operatorname{Sym}^{2}(\mathbf{2}) \oplus\left(\begin{array}{l}
n \\
2
\end{array}\right)(\mathbf{2} \otimes \mathbf{2})
$$

is completely reducible as an $\mathrm{SL}_{2}$-module. Hence the canonical map

$$
\operatorname{Sym}^{2}(V \otimes \mathbf{2})^{\mathrm{SL}_{2}} \longrightarrow \operatorname{Sym}^{2}(V \otimes \mathbf{2})_{\mathrm{SL}_{2}}
$$

from the invariants to the coinvariants, is an isomorphism of $\mathrm{SL}(V)$-modules. From (15), both modules are isomorphic to $\Lambda^{2} V$.

Returning to our task, we now require a $\operatorname{Spin}_{8}$-equivariant mapping

$$
\Lambda^{2} \mathbf{8} \rightarrow \Lambda^{2} \mathbf{8}^{\prime}
$$

But both of these exterior squares are isomorphic to the adjoint representation of $\mathrm{Spin}_{8}$, whence the desired invariant.

\subsubsection{Example: $E_{8}$ no. $4_{e}$}

The Kac diagram is

$$
\begin{array}{llllllll}
1 & 0 & 0 & 0 & 0 & 0 & 1 & 0
\end{array}
$$

0

with $G_{0}^{s c}=\operatorname{Spin}(12) \times \mathrm{SL}_{2}$ and

$$
\mathfrak{g}_{1}^{*}=(32 \otimes 1) \oplus(12 \otimes 2),
$$

where 32 is one of the half-spin representations of $\operatorname{Spin}_{12}$, which is symplectic since $6 \equiv 2 \bmod 4$. The action of the center of $G_{0}$ leads us to seek an invariant in

$$
\operatorname{Sym}^{2}(32 \otimes 1) \otimes \operatorname{Sym}^{2}(12 \otimes 2) .
$$

We must therefore find a $\operatorname{Spin}_{12} \times \mathrm{SL}_{2}$-equivariant mapping

$$
\operatorname{Sym}^{2}(12 \otimes 2) \longrightarrow \operatorname{Sym}^{2}(32 \otimes 1) .
$$

From (15) and (16) this is equivalent to a $\operatorname{Spin}_{12}$-equivariant mapping

$$
\Lambda^{2} 12 \longrightarrow \operatorname{Sym}^{2}(32)
$$

But $\Lambda^{2} \mathbf{1 2} \simeq \mathfrak{s o}_{12}$ and $\operatorname{Sym}^{2}(\mathbf{3 2}) \simeq \mathfrak{s p}_{32}$. The desired mapping $\mathfrak{s o}_{12} \rightarrow \mathfrak{s p}_{32}$ is simply the representation of $\mathfrak{s o}_{12}$ on the symplectic half-spin representation 32 . 


\subsection{A remark on saturation}

Let

$$
W^{*}(\mathfrak{c}, \theta):=N_{G^{\theta}}(\mathfrak{c}) / C_{G^{\theta}}(\mathfrak{c}) .
$$

Clearly $W(\mathfrak{c}, \theta) \subset W^{*}(\mathfrak{c}, \theta) \subset W_{1}^{\theta}$ (see (12)). We say that $\theta$ is saturated if $W(\mathfrak{c}, \theta)=W^{*}(\mathfrak{c}, \theta)$. (For the adjoint group $G$ this is equivalent to the definition given in section 5 of [35].) Clearly $\theta$ is saturated if $G^{\vartheta}=G_{0}$. As remarked in section 7.3 this holds whenever the group $\Omega_{\vartheta}(x)$ is trivial. In particular, saturation holds in types $G_{2},{ }^{3} D_{4}, F_{4}, E_{8},{ }^{2} E_{6}$, where $\Omega_{\vartheta}$ itself is trivial. It is known ([35], [19]) that all gradings on classical Lie algebras are saturated except for certain outer automorphisms of order divisible by 4 in type $D_{n}$. It remains to consider only those inner automorphisms of $E_{6}$ and $E_{7}$ where the Kac diagram is invariant under the symmetries of the affine Dynkin diagram and we have $W(\theta, \mathfrak{c}) \neq W_{1}^{\theta}$. The latter implies that $\left|C_{W_{J}}(w)\right|<c_{J}(w)$. The only cases not thus eliminated are $4_{d}$ and $4_{e}$ in type $E_{7}$. But in these two cases we have $c_{J}(w) /|W(\theta, \mathfrak{c})|=3$, while $\left[G^{\theta}: G_{0}\right]=2$, so saturation holds in these cases as well. We conclude that all gradings on exceptional Lie algebras are saturated.

\subsection{Kostant sections and the Levi subgroup $L_{\theta}$}

A Kostant section ${ }^{2}$ for the grading $\mathfrak{g}=\oplus_{i \in \mathbb{Z} / m} \mathfrak{g}_{i}$ is an affine subspace $\mathfrak{v} \subset \mathfrak{g}_{1}$ such that the embedding $\mathfrak{v} \hookrightarrow \mathfrak{g}_{1}$ induces an isomorphism of affine varieties $\mathfrak{v} \stackrel{\sim}{\longrightarrow} \mathfrak{g}_{1} / / G_{0}$, or equivalently, if the restriction map $k\left[\mathfrak{g}_{1}\right]^{G_{0}} \longrightarrow k[\mathfrak{v}]$ is bijective.

Recall that we have fixed a pinning $\left(X, R, \check{X}, \check{R},\left\{E_{i}\right\}\right)$ in $G$, which determines the co-character $\check{\rho} \in$ $X_{*}(T)$ and principal nilpotent element $E=\sum E_{i}$, such that $\check{\rho}(t) \cdot E=t E$. From [23, Thm.3.5] and $[19$, Prop.5.2] we have the following existence result for Kostant sections.

Theorem 10.2 Assume the characteristic of $k$ is not a torsion prime for $G$, that $m$ nonzero in $k$. Then the grading $\mathfrak{g}=\oplus_{i \in \mathbb{Z} / m} \mathfrak{g}_{i}$ associated to the principal automorphism $\theta_{m}=\check{\rho}(\zeta) \vartheta$ has a Kostant section $E+\mathfrak{u}$, where $\mathfrak{u}$ is any vector space complement to $\left[\mathfrak{g}_{0}, E\right]$ in $\mathfrak{g}_{1}$ such that $\mathfrak{u}$ is stable under $\check{\rho}\left(k^{\times}\right)$.

We have seen that for each positive-rank torsion inner automorphism in type $E_{6,7,8}$ there exists a subset $J \subseteq\{1,2, \ldots, \ell\}$ such that $W(\mathfrak{c}, \theta)=C_{W_{J}}(w)$. This can also be checked for the classical groups and types $F_{4}, G_{2}$. Thus, we have a case-by-case proof of the following theorem.

Theorem 10.3 Let $\theta$ be an inner automorphism of $\mathfrak{g}$ whose order $m$ is nonzero in $k$ and let $\mathfrak{c}$ be a Cartan subspace of $\mathfrak{g}_{1}$. Then there exists a $\theta$-stable Levi subgroup $L=L_{\theta}$ whose Lie algebra $\mathfrak{l}$ contains $\mathfrak{c}$ in its derived subalgebra, such that the following hold:

1. $\left.\theta\right|_{\mathfrak{l}}=\operatorname{Ad}\left(\check{\rho}_{L}(\zeta)\right)$.

\footnotetext{
${ }^{2}$ In the literature, this is also called a "Kostant-Weierstrass" or "KW" section because in the case of the non-pinned outer triality automorphism of $\mathfrak{s o}_{8}$ such a section is equivalent to the Weierstrass-normal form of a nonsingular homogeneous cubic polynomial in three variables.
} 
2. The inclusion of little Weyl groups $W_{L}(\mathfrak{c}, \theta) \hookrightarrow W(\mathfrak{c}, \theta)$ is a bijection. In particular, the degrees of $W(\mathfrak{c}, \theta)$ are precisely the degrees of $W_{L}$ which are divisible by $m$.

3. The restriction map $k\left[\mathfrak{g}_{1}\right]^{G_{0}} \longrightarrow k\left[\mathfrak{l}_{1}\right]^{L_{0}}$ is a bijection.

In view of Thm. 10.2, we conclude:

Corollary 10.4 Every positive-rank torsion inner automorphism in type $E_{6,7,8}$ has a Kostant section contained in the Levi subalgebra $\mathfrak{l}$ of the previous theorem.

We also observe:

Corollary 10.5 A positive integer $m$ is the order of a torsion inner automorphism of positive rank precisely if $m$ is the order of a $\mathbb{Z}$-regular element in the Weyl group of a Levi subgroup of $G$.

\section{Outer gradings of positive rank in type $E_{6}$}

We realize the outer pinned automorphism of $E_{6}$ as the restriction of an affine pinned automorphism of $E_{7}$, as in section 6 .

\subsection{Root systems of type $E_{7}$ and ${ }^{2} E_{6}$}

Let $(Y, R, \check{Y}, \check{R})$ be a root datum of adjoint type $E_{7}$ and fix a base $\Delta=\left\{\alpha_{1}, \ldots, \alpha_{7}\right\} \subset R$ with lowest root $\alpha_{0}$, according to the numbering

$$
\begin{array}{lllllll}
0 & 1 & 2 & 3 & 5 & 6 & 7
\end{array} .
$$

The set $\Pi:=\left\{\alpha_{0}\right\} \cup \Delta$ has stabilizer $W_{\Pi}=\{1, \vartheta\}$ of order two, where $\vartheta=r_{1} r_{2} r_{3}$ is a product of reflections about mutually orthogonal roots $\gamma_{1}, \gamma_{2}, \gamma_{3}$ in which the coefficients of simple roots $\left\{\alpha_{1}, \ldots, \alpha_{7}\right\}$ are given by

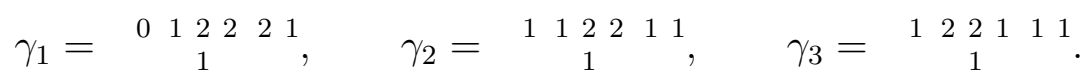

The sum

$$
\check{\gamma}_{1}+\check{\gamma}_{2}+\check{\gamma}_{3}=2 \check{\mu},
$$

where $\check{\mu}=\check{\omega}_{7}$ is the nontrivial minuscule co-weight.

Regard the vector space $V=\mathbb{R} \otimes \check{Y}$ as an affine space with 0 as basepoint. Each linear functional $\lambda: V \rightarrow \mathbb{R}$ is then regarded as an affine function on $V$ vanishing at 0 , we have the affine root system

$$
\Phi=\{\alpha+n: \alpha \in R, n \in \mathbb{Z}\}
$$

with basis $\left\{\phi_{0}, \phi_{1}, \ldots, \phi_{7}\right\}$ where $\phi_{0}=1+\alpha_{0}, \phi_{1}=\alpha_{1}, \ldots, \phi_{7}=\alpha_{7}$ satisfy the relation

$$
\phi_{0}+2 \phi_{1}+3 \phi_{2}+4 \phi_{3}+2 \phi_{4}+3 \phi_{5}+2 \phi_{6}+\phi_{7}=1 \text {. }
$$


A point $x \in V_{\mathbb{Q}}$ of order $m$ has Kac diagram

$$
\begin{array}{lllllll}
s_{0} & s_{1} & s_{2} & s_{3} & s_{5} & s_{6} & s_{7}
\end{array},
$$

where $s_{i} / m=\phi_{i}(x)$.

The affine transformation $\widetilde{\vartheta}: V \rightarrow V$ given by

$$
\widetilde{\vartheta}(x)=\check{\mu}+\vartheta \cdot x
$$

permutes the simple affine roots $\left\{\phi_{0}, \ldots, \phi_{7}\right\}$ according to the nontrivial symmetry of the affine diagram of $E_{7}$. The fixed-point space of $\widetilde{\vartheta}$ in $V$ is given by

$$
\mathcal{A}^{\vartheta}:=V^{\vartheta}+\frac{1}{2} \check{\mu}
$$

which is an affine space under the vector space $V^{\vartheta}=\mathbb{R} \otimes \check{Y}_{\vartheta}$, with basepoint $\frac{1}{2} \check{\mu}$. The rational points in $\mathcal{A}^{\vartheta}$ are precisely those points $x \in V_{\mathbb{Q}}$ whose Kac diagram has the symmetric form

$$
\begin{array}{lllllll}
s_{0} & s_{1} & s_{2} & s_{3} & s_{2} & s_{1} & s_{0}
\end{array},
$$

in which case equation (18) implies that

$$
s_{0}+2 s_{1}+3 s_{2}+2 s_{3}+s_{4}=m / 2
$$

where $m$ is the order of $x$.

The automorphism $\vartheta$ permutes the roots $\alpha_{1}, \ldots, \alpha_{6}$ which generate a root subsystem $R^{\prime}$ of type $E_{6}$. The co-weight lattice $\check{X}=\operatorname{Hom}\left(\mathbb{Z} R^{\prime}, \mathbb{Z}\right)$ has dual basis $\left\{\check{\omega}_{1}, \ldots, \check{\omega}_{6}\right\}$ and we have

$$
\check{X}^{\vartheta}=\check{Y}^{\vartheta}
$$

Hence $\mathcal{A}^{\vartheta}$ is also an affine space under $\mathbb{R} \otimes \check{X}^{\vartheta}$ and we may construct the affine root system $\Psi\left(R^{\prime}, \vartheta\right)$ as in section 2.1, using the point $x_{0}=\frac{1}{2} \check{\mu}$. We have $\ell_{\vartheta}=4$ and $\Psi\left(R^{\prime}, \vartheta\right)$ has basis $\psi_{0}, \ldots, \psi_{4}$, where $\psi_{i}=\left.\alpha_{i}\right|_{\mathcal{A}^{\vartheta}}$ for $1 \leq i \leq 4$ and

$$
\psi_{0}+2 \psi_{1}+3 \psi_{2}+2 \psi_{3}+\psi_{4}=1 / 2
$$

A rational point $x \in \mathcal{A}_{\mathbb{Q}}^{\vartheta}$ with $E_{7}$ Kac-diagram (20) has ${ }^{2} E_{6}$ Kac-diagram

$$
s_{0} s_{1} s_{2} \Leftarrow s_{3} s_{4} .
$$

This is clear for $s_{1}, \ldots, s_{4}$ since $\psi_{i}$ is the restriction of $\phi_{i}$, and follows for $s_{0}$ by comparing the relations (18) and (22). 


\subsection{Lie algebras of type $E_{7}$ and ${ }^{2} E_{6}$}

Let $k$ be an algebraically closed field of characteristic $\neq 2,3$ and let $\mathfrak{g}$ be a simple Lie algebra over $k$ of type $E_{7}$ with automorphism group $G=\operatorname{Aut}(\mathfrak{g})$. We fix a maximal torus $T \subset G$ with Lie algebra $\mathfrak{t}$ and we choose an affine pinning $\widetilde{\Pi}=\left\{E_{0}, \ldots, E_{7}\right\}$ for $T$ in $\mathfrak{g}$, numbered as in (17). As above we let $\vartheta=r_{1} r_{2} r_{3} \in W_{\Pi}$ be the unique involution acting on $\Pi$ via the permutation $(07)(16)(25)$. Recall from section 6 that $\vartheta$ has a lift $n \in N$ of order two defined via the homomorphism $\varphi: \mathrm{SL}_{2} \rightarrow G$ as in equation (11).

Let $S=\left(T^{\vartheta}\right)^{\circ}$ be the identity component of the group of fixed-points of $\vartheta$ in $T$. The co-weight group of $S$ is $\check{X}^{\vartheta}$ and we have

$$
T^{\vartheta}=S \times\langle\check{\mu}(-1)\rangle,
$$

where $\check{\mu}=\check{\omega}_{7}$ is the nontrivial minuscule co-weight. The automorphism $\varepsilon:=\operatorname{Ad}(\check{\mu}(-1))$ has order two; its fixed-point subalgebra $\mathfrak{g}^{\varepsilon}$ decomposes as

$$
\mathfrak{g}^{\varepsilon}=\mathfrak{h} \oplus \mathfrak{z}
$$

where $\mathfrak{z}=d \check{\mu}(k)$ and $\mathfrak{h}=\left[\mathfrak{g}^{\varepsilon}, \mathfrak{g}^{\varepsilon}\right]$, the derived subalgebra of $\mathfrak{g}^{\varepsilon}$, has type $E_{6}$ and is generated by the root spaces $\mathfrak{g}_{\alpha}$ for $\alpha \in \pm\left\{\alpha_{1}, \ldots, \alpha_{6}\right\}$. Note that $\varepsilon$ and $n$ both lie in the subgroup $\varphi\left(\mathrm{SL}_{2}\right)$ and are conjugate therein.

The centralizer $C_{G}(\varepsilon)$ is the normalizer in $G$ of $\mathfrak{h}$, surjecting onto $\operatorname{Aut}(\mathfrak{h})$, and is also the normalizer of in $G$ of $\mathfrak{z}$. The centralizer $C_{G}(\mathfrak{z})$ of $\mathfrak{z}$ is the identity component of $C_{G}(\varepsilon)$, and the image of $C_{G}(\mathfrak{z})$ in $\operatorname{Aut}(\mathfrak{h})$ is the group

$$
H:=\operatorname{Aut}(\mathfrak{h})^{\circ}
$$

of inner automorphisms of $\mathfrak{h}$. It follows that we have an exact sequence

$$
1 \longrightarrow \mu\left(k^{\times}\right) \longrightarrow C_{G}(\mathfrak{z}) \longrightarrow H \longrightarrow 1
$$

Proposition 11.1 Let $\theta \in \operatorname{Aut}(\mathfrak{g})$ be a torsion automorphism whose order $m$ is nonzero in $k$. Then the centralizer $G^{\theta}$ has at most two components, and the following are equivalent.

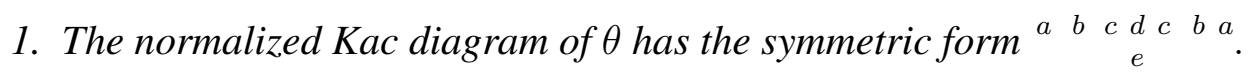

2. The G-conjugacy class of $\theta$ meets $S n$.

3. The centralizer $G^{\theta}$ has two components and $n$ lies in the non-identity component.

Proof: After conjugating by $G$, we may assume $\theta=\operatorname{Ad}(t)$, where $t=\check{\lambda}(\zeta)$, for some $\check{\lambda} \in \check{X}$ and $\zeta \in k^{\times}$of order $m$. We set $x=\frac{1}{m} \check{\lambda}$.

Over $\mathbb{C}$, the equivalence $1 \Leftrightarrow 3$ follows from [24, Prop. 2.1], whose proof, once we replace $\exp (x)$ by $\check{\lambda}(\zeta)$, is also valid over $k$.

We prove $1 \Leftrightarrow 2$. From the previous section the Kac coordinates of $\theta$ are symmetric precisely if

$$
x=\check{\mu}+\vartheta \cdot x .
$$


This is equivalent to having $\check{\lambda}-\frac{m}{2} \check{\mu} \in \check{X}^{\vartheta}$. Evaluating at $\zeta$ this is in turn equivalent to having $t \varepsilon \in S$, or $t \in S \varepsilon$. Since $n$ and $\varepsilon$ are conjugate in $\varphi\left(\mathrm{SL}_{2}\right)$ which centralizes $S$ (see Lemma 6.3) we can replace $\varepsilon$ by $n$.

Proposition 11.2 Let $s \in S$ and suppose sn has order $m$ invertible in $k$ and let $\theta=\operatorname{Ad}(s n)$ have

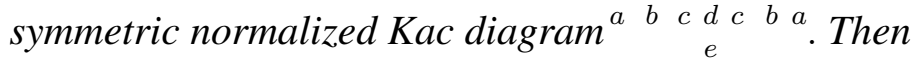

1. $\theta$ normalizes $\mathfrak{h}$ and $\left.\theta\right|_{\mathfrak{h}}$ is an outer automorphism of $\mathfrak{h}$ with Kac diagram

$$
a b c \Leftarrow d e .
$$

2. Every torsion outer automorphism of $\mathfrak{h}$ is conjugate to $\left.\theta\right|_{\mathfrak{h}}$, where $\theta=\operatorname{Ad}($ sn $)$ for some $s \in S$.

3. We have $\operatorname{rank}\left(\left.\theta\right|_{\mathfrak{h}}\right) \leq \operatorname{rank}(\theta)$.

Proof: Since $\operatorname{Ad}(n)=\vartheta$ normalizes $\mathfrak{h}$, acting there via a pinned automorphism, and $s \in S \subset H$, we have that $\left.\theta\right|_{\mathfrak{h}}$ is an outer automorphism of $\mathfrak{h}$. The relation between the Kac diagrams of $\theta$ and $\left.\theta\right|_{\mathfrak{h}}$ follows from the discussion in section 11.1.

Assertion 2 is now clear, since every Kac diagram $s_{0} s_{1} s_{2} \Leftarrow s_{3} s_{4}$ corresponds to $\left.\operatorname{Ad}(s n)\right|_{\mathfrak{h}}$ for some $s \in S$. We can also prove assertion 2 directly, as follows: Since $\vartheta$ preserves the maximal torus $T \cap H$ of $H$, and permutes the simple roots $\left\{\alpha_{1}, \ldots, \alpha_{6}\right\}$, every torsion outer automorphism of $\mathfrak{h}$ is $H$-conjugate to one of the form $\operatorname{Ad}(s) \vartheta$ for some $s \in(T \cap H)^{\vartheta}$ (see [24, Lemma 3.2], whose proof is valid for $k$ ). We must therefore show that $(T \cap H)^{\vartheta}=S$. Since the Lie algebra of $S$ is $\mathfrak{t}^{\vartheta}$ which is contained in $(\mathfrak{t} \cap \mathfrak{h})^{\vartheta}$, it suffices to show that $\mathfrak{t}^{\vartheta} \subset \mathfrak{h}$. But $\mathfrak{t}^{\vartheta}$ has dimension four and is spanned by $d \check{\alpha}_{i}(1)+d \check{\alpha}_{7-i}(1)$ for $1 \leq i \leq 4$, and these vectors lie in $\mathfrak{h}$.

Finally, a Cartan subspace for $\left.\theta\right|_{\mathfrak{h}}$ is contained in a Cartan subspace for $\theta$, so assertion 3 is obvious.

Prop. 11.2 implies that the Kac diagram of any outer positive rank automorphism of $\mathfrak{h}$ must have the form $a b c \Leftarrow d e$, where $\begin{array}{ccc}a b & c & b \\ e & \end{array}$ is a positive rank diagram for $E_{7}$ appearing in section 9.2.

For example, there are two outer automorphisms of $\mathfrak{h}$ having order $m=2$, namely the restrictions to $\mathfrak{h}$ of $\vartheta=\operatorname{Ad}(n)$ and $\vartheta_{0}=\operatorname{Ad}\left(n_{0}\right)$ where $n_{0}$ is a lift of $-1 \in W\left(E_{7}\right)$. These are the involutions in $E_{7}$ numbered $2_{c}$ and $2_{a}$ respectively Table 20 of section 9.2. The Kac diagrams in $E_{7}$ and ${ }^{2} E_{6}$ are shown:

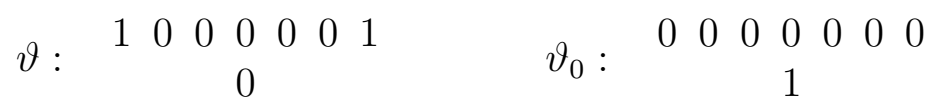

$$
\begin{aligned}
& \left.\vartheta\right|_{\mathfrak{h}}:\left.\quad 100 \Leftarrow 00 \quad \vartheta_{0}\right|_{\mathfrak{h}}: \quad 000 \Leftarrow 01 .
\end{aligned}
$$

Both $\vartheta$ and $\vartheta_{0}$ act by -1 on $\mathfrak{z}$. It follows that their ranks in $E_{6}$ are one less than their ranks in $E_{7}$, namely

$$
\operatorname{rank}\left(\left.\vartheta\right|_{\mathfrak{h}}\right)=2, \quad \operatorname{rank}\left(\left.\vartheta_{0}\right|_{\mathfrak{h}}\right)=6
$$




\subsection{Positive rank gradings on $E_{6}$ (outer case)}

From Props. 11.1 and 11.2 we know that the Kac diagrams for positive rank gradings in outer type $E_{6}$ are obtained from symmetric positive-rank diagrams for $E_{7}$. We now adapt our methods for the inner case to complete the classification of positive rank outer gradings of $E_{6}$.

We regard $W\left(E_{6}\right)$ as the subgroup of $W\left(E_{7}\right)$ generated by the reflections for the roots $\alpha_{1}, \ldots, \alpha_{6}$. Equivalently, $W\left(E_{6}\right)$ is the centralizer of $\mathfrak{z}$ in $W\left(E_{7}\right)$. The coset $-W\left(E_{6}\right)=\left\{w \vartheta_{0}: w \in W\left(E_{6}\right)\right\}$ consists of the elements in $W\left(E_{7}\right)$ acting by -1 on $\mathfrak{z}$ and contains both $\vartheta$ and $\vartheta_{0}$.

Lemma 11.3 Let $n_{w} \in N_{G}(\mathfrak{t})$ be a lift of an element $w \in-W\left(E_{6}\right)$. Then $\operatorname{Ad}\left(n_{w}\right)$ normalizes $\mathfrak{h}$ and acts on $\mathfrak{h}$ as an outer automorphism.

Proof: Since $w$ permutes the root spaces in $\mathfrak{h}$ it follows that $n_{w}$ normalizes $\mathfrak{h}$. Let $n \in N_{G}(\mathfrak{t})$ be the lift of $\vartheta$ constructed above. Both $n$ and $n_{w}$ act by -1 on $\mathfrak{z}$, so $n \cdot n_{w}$ lies in the connected subgroup $C_{G}(\mathfrak{z})$ and the image of $n \cdot n_{w}$ in $\operatorname{Aut}(\mathfrak{h})$ lies in the subgroup $\operatorname{Aut}(\mathfrak{h})^{\circ}$ of inner automorphisms. Since $\operatorname{Ad}(n)=\vartheta$ is outer on $\mathfrak{h}$, it follows that $\operatorname{Ad}\left(n_{w}\right)$ is outer on $\mathfrak{h}$ as well.

Let $w \in W\left(E_{7}\right)$ be any element whose order $m$ is invertible in $k$ and such that $w$ has an eigenvalue $\zeta$ of order $m$ on $\mathfrak{t}$. Recall that $\operatorname{Kac}(w)$ is the set of normalized Kac diagrams of torsion automorphisms $\theta \in \operatorname{Aut}(\mathfrak{g})$ of order $m$ such that $\theta$ normalizes $\mathfrak{t}$ and acts on $\mathfrak{t}$ via $w$.

Let $\tau \in \operatorname{Aut}(\mathfrak{h})$ be a torsion outer automorphism with Kac coordinates $a b c \Leftarrow d e$. We write

$$
\tau \rightsquigarrow w
$$

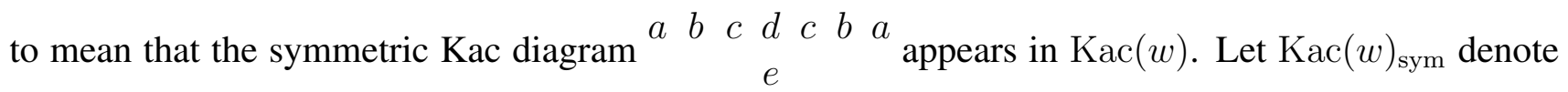
the set of symmetric diagrams in $\operatorname{Kac}(w)$.

Proposition 11.4 Let $\tau \in \operatorname{Aut}(\mathfrak{h})$ be a torsion outer automorphism whose order $m>2$ is invertible in $k$. Assume that $\operatorname{rank}(\tau)>0$. Then there exists $w \in-W\left(E_{6}\right)$ such $\tau \rightsquigarrow w$. Moreover, we have

$$
\operatorname{rank}(\tau)=\max \left\{\operatorname{rank}(w): w \in-W\left(E_{6}\right), \tau \rightsquigarrow w\right\}
$$

Proof: Let $\mathfrak{c} \subset \mathfrak{h}(\tau, \zeta)$ be a Cartan subspace. Then $\mathfrak{c}$ is contained in a $\tau$-stable Cartan subalgebra $\mathfrak{t}^{\prime}$ of $\mathfrak{h}$ so that $\mathfrak{c}=\mathfrak{t}^{\prime}(\tau, \zeta)$. Conjugating by $H$, we may assume that $\mathfrak{t}^{\prime} \subset \mathfrak{t}$ and therefore $\mathfrak{t}=\mathfrak{t}^{\prime} \oplus \mathfrak{z}$.

We have $\tau=\left.\theta\right|_{\mathfrak{h}}$ for some $\theta \in \operatorname{Aut}(\mathfrak{g})$ normalizing $\mathfrak{h}$. Then $\theta$ also normalizes the centralizer $\mathfrak{z}$ of $\mathfrak{h}$. Since $\left.\theta\right|_{\mathfrak{h}}$ is outer but $\left.\theta^{2}\right|_{\mathfrak{h}}$ is inner, it follows that $\theta$ acts by -1 on $\mathfrak{z}$.

Since $\theta$ normalizes $\mathfrak{t}$, it projects to an element $w \in W\left(E_{7}\right)$. The subgroup of $W\left(E_{7}\right)$ normalizing $\mathfrak{z}$ is $\{ \pm 1\} \times W\left(E_{6}\right)$ and $W\left(E_{6}\right)$ is the subgroup centralizing $\mathfrak{z}$. It follows that $w \in-W\left(E_{6}\right)$.

Since the normalized $\operatorname{Kac}$ diagram of $\theta$ belongs to $\operatorname{Kac}(w)$ and $\tau=\left.\theta\right|_{\mathfrak{h}}$, we have $\tau \rightsquigarrow w$. We also have

$$
\operatorname{rank}(w)=\mathfrak{t}(w, \zeta)=\mathfrak{t}^{\prime}(w, \zeta)
$$


Suppose now that $w \in-W\left(E_{6}\right)$ is any element for which $\tau \rightsquigarrow w$. Let $a b c \Leftarrow d e$ be the normalized Kac coordinates for $\tau$. Since $\tau \rightsquigarrow w$ there is a lift $n_{w} \in N_{G}(\mathfrak{t})$ such that $\operatorname{Ad}\left(n_{w}\right)$ has normalized $\operatorname{Kac}$ diagram $\begin{array}{ccccccc}a & b & c & d & c & b & a \\ & e & & & & \end{array}$.

By Lemma (11.3), we have that $\operatorname{Ad}\left(n_{w}\right)$ is an outer automorphism of $\mathfrak{h}$. Hence there is $s \in S$ such that $\left.\operatorname{Ad}\left(n_{w}\right)\right|_{\mathfrak{h}}$ is $H$-conjugate to $\left.\operatorname{Ad}(s n)\right|_{\mathfrak{h}}$. From the exact sequence (23) there are $g \in C_{G}(\mathfrak{z})$ and $z \in Z$ such that

$$
g n_{w} z g^{-1}=s n \text {. }
$$

But $n_{w}$ is $Z$-conjugate to $n_{w} z$, since $w=-1$ on $\mathfrak{z}$. Therefore $n_{w}$ and $s n$ are conjugate under $C_{G}(\mathfrak{z})$, so

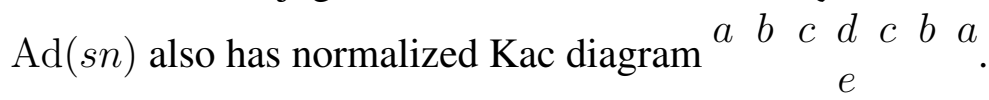

By Prop. 11.2, $\left.\operatorname{Ad}(s n)\right|_{\mathfrak{h}}$ has Kac diagram $a b c \Leftarrow d e$, and therefore $\left.\operatorname{Ad}(s n)\right|_{\mathfrak{h}}$ is $H$-conjugate to $\tau$. But

$$
\left.\operatorname{Ad}(s n)\right|_{\mathfrak{h}}=\left.\operatorname{Ad}\left(g n_{w} z g^{-1}\right)\right|_{\mathfrak{h}}=\left.\operatorname{Ad}\left(g n_{w} g^{-1}\right)\right|_{\mathfrak{h}}
$$

is conjugate to $\left.\operatorname{Ad}\left(n_{w}\right)\right|_{\mathfrak{h}}$, via the element $h=\left.\operatorname{Ad}(g)\right|_{\mathfrak{h}} \in H$. Thus, $\tau$ and $\left.\operatorname{Ad}\left(n_{w}\right)\right|_{\mathfrak{h}}$ are $H$-conjugate. Since $\mathfrak{t}(w, \zeta) \subset \mathfrak{h}$, an $H$-conjugate of $\mathfrak{t}(w, \zeta)$ is contained in a Cartan subspace of $\tau$, so $\operatorname{rank}(w) \leq$ $\operatorname{rank}(\tau)$. This completes the proof.

The Kac diagrams of positive rank for ${ }^{2} E_{6}$ are obtained from symmetric positive rank diagrams for $E_{7}$, of which there are 20 (see Table 20).

Three of these $\left(14_{a}, 8_{d}, 8_{e}\right)$ have rank zero for ${ }^{2} E_{6}$ as will be explained. Two more have order $m=2$ and are easily handled by known results. The ranks for the remaining 15 are found as follows. Using Prop. 11.4, it is enough to extract the symmetric diagrams from the preliminary table for $E_{7}$ in section 9.1. The results are shown below, where $r$ is the rank of $\tau$ in ${ }^{2} E_{6}$.

\begin{tabular}{|c|c|c|c|c|c|c|c|}
\hline$m$ & $w \in-W\left(E_{6}\right)$ & $w \in W\left(E_{7}\right)$ & $r$ & $\operatorname{Kac}(w)_{\text {un }}$ & $\operatorname{Kac}(w)_{\mathrm{sym}}$ & & \\
\hline 18 & $-E_{6}\left(a_{1}\right)$ & $E_{7}$ & 1 & $\begin{array}{llllllll}1 & 1 & 1 & 1 & 1 & 1 & 1 \\
& & & 1 & & & \end{array}$ & \begin{tabular}{|llllllll} 
& 1 & 1 & 1 & 1 & 1 & 1 & 1 \\
& & & 1 & & &
\end{tabular} & & \\
\hline 12 & $-E_{6}$ & $E_{7}\left(a_{2}\right)$ & 1 & $\begin{array}{ccccccc}1 & 1 & 0 & 1 & 0 & 1 & 1 \\
& & & 1 & & & \end{array}$ & $\begin{array}{lllllll}1 & 1 & 0 & 1 & 0 & 1 & 1 \\
& & & 1 & & & \end{array}$ & & \\
\hline 10 & $-\left(A_{4}+A_{1}\right)$ & $D_{6}$ & 1 & $\begin{array}{ccccccc}* & * & 1 & 1 & 1 & 1 & 1 \\
& & 1 & & & \end{array}$ & $\begin{array}{llllllll} & 0 & 1 & 0 & 1 & 0 & 1 & 0 \\
& & & 1 & & & \end{array}$ & 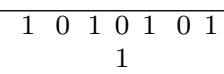 & $\begin{array}{lllllll} & 1 & 0 & 1 & 0 & 1 & 1\end{array}$ \\
\hline 8 & $-D_{5}$ & $D_{5}+A_{1}$ & 1 & $\begin{array}{cccccc}1 & * & 1 & 1 & 1 & 1\end{array}$ & \begin{tabular}{|llllllll}
0 & 1 & 0 & 1 & 0 & 1 & 0 \\
& & & 0 & & & \\
\end{tabular} & $\begin{array}{lllllll}1 & 0 & 0 & 1 & 0 & 0 & 1 \\
& & & 1 & & & \end{array}$ & \\
\hline 6 & $-\left(3 A_{2}\right)$ & $E_{7}\left(a_{4}\right)$ & 3 & $\begin{array}{ccccccc}1 & 0 & 0 & 1 & 0 & 0 & 1 \\
& & & 0 & & & \\
\end{array}$ & \begin{tabular}{|lllllll}
1 & 0 & 0 & 1 & 0 & 0 & 1 \\
& & & 0 & & & \\
\end{tabular} & & \\
\hline 6 & $-\left(2 A_{2}\right)$ & $A_{1}+D_{6}\left(a_{2}\right)$ & 2 & $\begin{array}{ccccccc}1 & * & 1 & 0 & 1 & 0 & 1 \\
& & & & \end{array}$ & $\begin{array}{lllllllll}0 & 1 & 0 & 0 & 0 & 1 & 0 \\
& & & 1 & & & \end{array}$ & $\begin{array}{lllllll}1 & 0 & 0 & 1 & 0 & 0 & 1\end{array}$ & \\
\hline 6 & $-A_{2}$ & $3 A_{1}+D_{4}$ & 1 & $\begin{array}{ccccccc}1 & * & 1 & 1 & 1 & * & * \\
1 & & & \end{array}$ & $\begin{array}{llllllll}0 & 0 & 0 & 1 & 0 & 0 & 0 \\
& & & 1 & & & \end{array}$ & & \\
\hline 6 & $-\left(A_{1}+A_{5}^{\prime \prime}\right)$ & $A_{5}^{\prime}$ & 1 & $\begin{array}{cccccc}* & 1 & 1 & 1 & 1 & 1\end{array}$ & \begin{tabular}{|ccccccc}
0 & 0 & 1 & 0 & 1 & 0 & 0 \\
& & & 0 & & & \\
1 & 0 & 0 & 1 & 0 & 0 & 1 \\
& & & 0 & & & \\
\end{tabular} & $\begin{array}{lllllll}1 & 1 & 0 & 0 & 0 & 1 & 1 \\
& & & 0 & & & \end{array}$ & 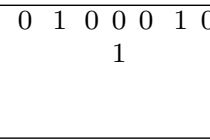 \\
\hline 4 & $-D_{4}\left(a_{1}\right)$ & $A_{1}+2 A_{3}$ & 2 & 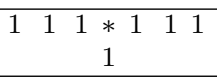 & \begin{tabular}{|lllllll}
0 & 0 & 0 & 1 & 0 & 0 & 0 \\
& & & 0 & & & \\
\end{tabular} & & \\
\hline 4 & $-A_{3}+2 A_{1}$ & $\left(A_{1}+A_{3}\right)^{\prime \prime}$ & 1 & 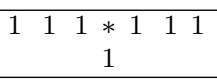 & 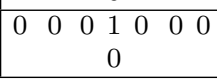 & 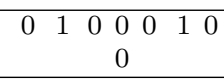 & $\begin{array}{ccccccc}1 & 0 & 0 & 0 & 0 & 0 & 1 \\
& & & & 1 & & \\
\end{array}$ \\
\hline
\end{tabular}

Table 22: $\operatorname{Kac}(w)_{\mathrm{sym}}$ for certain $w$ in $-W\left(E_{6}\right)$ 
Case $14_{a}$ has rank zero since there are no elements of order 7 or 14 in $W\left(E_{6}\right)$. Cases $8_{d}$ and $8_{e}$ have rank zero since $D_{5}$ is the only element of order 8 in $W\left(E_{6}\right)$ and the Kac diagrams for $8_{d, e}$ do not appear in the row for $w=-D_{5}$ in Table 22 above.

\subsection{Little Weyl groups for ${ }^{2} E_{6}$}

The little Weyl groups $W_{H}(\mathfrak{c}, \tau)$ and their degrees are determined as follows.

Cases $18_{a}, 12_{b}, 6_{a}, 4_{b}, 2_{a}$ : These cases are stable, hence by Cor. 7.4 we have $W_{H}(\mathfrak{c}, \tau)=W\left(\mathfrak{t}^{\prime}\right)^{\theta}$, where $\mathfrak{t}^{\prime}$ is the unique Cartan subalgebra of $\mathfrak{h}$ containing $\mathfrak{c}$. Then $W\left(\mathfrak{t}^{\prime}\right)^{\theta}$ and its degrees are determined from [29].

Lemma 11.5 If $\operatorname{dim} \mathfrak{c}=1$ then $W_{H}(\mathfrak{c}, \tau) \simeq \boldsymbol{\mu}_{d}$ for some integer d divisible by $m / 2$.

Proof: Since $\operatorname{dim} \mathfrak{c}=1$ we have $W_{H}(\mathfrak{c}, \tau) \simeq \boldsymbol{\mu}_{d}$ for some integer $d$. We may assume $\tau=\left.\operatorname{Ad}\left(n_{w}\right)\right|_{\mathfrak{h}}$, where $n_{w} \in N_{G}(\mathfrak{t})$ has image $w \in-W\left(E_{6}\right)$. Then $n_{w}^{2} \in H_{0}$ has eigenvalue $\zeta^{2}$ on $\mathfrak{c}$, where $\zeta \in k^{\times}$has order $m$ equal to the order of $\tau$. It follows that so $m / 2$ divides $d$.

Cases $10_{a}, 10_{b}, 10_{c}$ : In these cases we have $m=10$ and $\operatorname{dim} \mathfrak{c}=1$ so $\boldsymbol{\mu}_{5} \leq W_{H}(\mathfrak{c}, \tau)$, by Lemma 11.5. And $W_{H}(\mathfrak{c}, \tau) \leq W_{H}\left(\mathfrak{c}, \tau^{2}\right)$. Now $w^{2}$ has type $A_{4}$ in $E_{6}$, and all lifts of this type have little Weyl group $\boldsymbol{\mu}_{5}$, from Table 19. ${ }^{3}$ So And $W_{H}(\mathfrak{c}, \tau) \leq W_{H}\left(\mathfrak{c}, \tau^{2}\right) \simeq \boldsymbol{\mu}_{5}$.

Cases $8_{c}, 8_{f}:$ In these cases we have $m=8$ and $\operatorname{dim} \mathfrak{c}=1$ so $\boldsymbol{\mu}_{4} \leq W_{H}(\mathfrak{c}, \tau) \leq \boldsymbol{\mu}_{8}$, by Lemma 11.5.

In case $8_{f}$ the diagram for $\theta^{\prime}$ in Table 20 shows that $\tau$ is principle in $\operatorname{Aut}(\mathfrak{h})$. Hence $W_{H}(\mathfrak{c}, \tau)=$ $N_{W_{H}}(\mathfrak{c}) / Z_{W_{H}}(\mathfrak{c})$, by Prop. 7.2. The element $w$ has type $-D_{5}$ and $\mathfrak{c}$ may be chosen to be the $-\zeta-$ eigenspace for $y=-w$ in $\mathfrak{t}$. Since $\langle y\rangle$ acts faithfully on $\mathbb{C}$, there is a copy of $\boldsymbol{\mu}_{8}$ in $W_{H}(\mathfrak{c}, \tau)$.

In case $8_{c}$ we rule out $\boldsymbol{\mu}_{4}$ using invariant theory, as in section 10. A degree-four invariant in $\mathfrak{h}_{1}$ would correspond to an element of

$$
\operatorname{Hom}_{M}\left(\operatorname{Sym}^{2}(\mathbf{2} \otimes \mathbf{2})^{L}, \operatorname{Sym}^{2}(\mathbf{3} \otimes \mathbf{2})^{R}\right),
$$

arising from the action of $L \times M \times R=\mathrm{SL}_{2} \times \mathrm{SL}_{2} \times \mathrm{SL}_{2}$ on

$$
\mathfrak{h}_{1} \simeq 2 \otimes 2 \otimes 1 \oplus 1 \otimes 3 \otimes 2
$$

But $\operatorname{Sym}^{2}(2 \otimes \mathbf{2})^{L}$ is the trivial representation of $M$ and $\operatorname{Sym}^{2}(\mathbf{3} \otimes \mathbf{2})^{R}$ is the adjoint representation of $M$, which is irreducible since $p>2$. Hence the vector space (24) is zero.

Case $6_{c}$ : Here the centralizer of $w=-2 A_{2}$ in $W\left(E_{6}\right)$ has order 108 and contains a subgroup $W\left(A_{2}\right)$ acting trivially on the root subsystem spanned by the $2 A_{2}$. It follows that $\left|W_{H}(\tau, \mathfrak{c})\right| \leq 18$. Results in the next section show that $W_{H}(\tau, \mathfrak{c})$ contains the centralizer of a [33]-cycle in the symmetric group $S_{6}$, which has order 18 . 19.

${ }^{3}$ In fact, using Kac diagrams one can check that classes $10_{a, b, c}$ in Table 20 square to classes $5_{a, b, c}$, respectively, in Table 
Case $6_{g}$ : Here $\operatorname{dim} \mathfrak{c}=1$ and $w^{2}$ has type $A_{2}$, of which all lifts in $H$ have little Weyl group $\mu_{6}$. Hence $\mu_{3} \leq W_{H}(\mathfrak{c}, \tau) \leq \mu_{6}$. One checks that an $H_{0}$-invariant in degree 3 in $\mathfrak{h}_{1}$ is a quadratic form on $S^{2} \check{\mathbf{4}}$, which must be trivial. Hence $W_{H}(\mathfrak{c}, \tau) \simeq \mu_{6}$.

Cases $6_{i}, 6_{k}$ : These cases have $m=6$ and $\operatorname{dim} \mathfrak{c}=1$ so $\mu_{3} \leq W_{H}(\mathfrak{c}, \tau)$, by Lemma 11.5. We show this is equality by finding an $H_{0}$-invariant of degree 3 on $\mathfrak{h}_{1}$.

In case $6_{i}, \mathfrak{h}_{1}$ is the respresentation $3 \otimes \check{\mathbf{3}}=\operatorname{End}(\mathbf{3})$ of $\mathrm{SL}_{3} \times \mathrm{SL}_{3}$, and the determinant is a cubic invariant.

In case $6_{k}, \mathfrak{h}_{1}$ is the respresentation $1 \oplus \mathbf{8}$ of $\operatorname{Spin}_{7}$, where $\mathbf{8}$ is the Spin representation, which affords an invariant quadratic form $q$. The map $(x, v) \mapsto x \cdot q(v)$ is a cubic invariant.

Cases $4_{e}, 4_{d}$ : These cases have $m=4$ and $\operatorname{dim} \mathfrak{c}=1$ so $\mu_{2} \leq W_{H}(\mathfrak{c}, \tau)$, by Lemma 11.5. We show that in both cases there is a quartic invariant but no quadratic invariant.

In case $4_{e}, \mathfrak{h}_{1}$ is the representation $\Lambda^{3}(\mathbf{6})=\mathbf{6} \oplus \mathbf{1 4}$ of $\operatorname{Sp}_{6} \times T_{1}$, where $t \in T_{1}$ acts by $t, t^{-1}$ on the respective summands. Since $p>2$ both summands are irreducible so there is no invariant in bidegree $(1,1)$. In characteristic zero one computes that $\operatorname{Sym}^{2}(\mathbf{6})$ appears in $\operatorname{Sym}^{2}(\mathbf{1 4})$, giving a nonzero $H_{0}$ quartic invariant, which persists in positive characteristic by Lemma 10.1.

In case $4_{d}, \mathfrak{h}_{1}$ is the representation $2 \otimes 8$ of $\mathrm{SL}_{2} \times \mathrm{Spin}_{7}$. Since this representation is irreducible and symplectic there is no quadratic invariant. To find a quartic invariant we may assume the characteristic of $k$ is zero. Write

$$
\mathfrak{h}_{1}=\mathbf{8}_{+} \oplus \mathbf{8}_{-},
$$

according to the characters $t \mapsto t^{ \pm 1}$ of the maximal torus of $\mathrm{SL}_{2}$. One checks that

$$
\operatorname{dim}\left[\operatorname{Sym}^{4-i}\left(\mathbf{8}_{+}\right) \otimes \operatorname{Sym}^{i}\left(\mathbf{8}_{-}\right)\right]^{\operatorname{Spin}_{7}}= \begin{cases}1 & \text { for } i \neq 2 \\ 2 & \text { for } i=2\end{cases}
$$

Since this summand affords the character $t^{4-2 i}$ of the maximal torus of $S L_{2}$, it follows that there is a one-dimensional space of quartic invariants in $\mathfrak{h}_{1}$ for $\mathrm{SL}_{2} \times \mathrm{Spin}_{7}$.

\subsection{Standard subalgebras and Kostant sections}

Fix a torsion automorphism $\theta=\operatorname{Ad}(s) \vartheta$ of $\mathfrak{h}=\mathfrak{e}_{6}$, with $s \in S=\left(T^{\vartheta}\right)^{\circ}$, and let $\tau \in \operatorname{Aut}(\mathfrak{h})$ be another torsion automorphism of the form $\tau=\operatorname{Ad}(t)$ (inner case) or $\tau=\operatorname{Ad}(t) \vartheta$ (outer case), for some $t \in S$. We call the fixed-point subalgebra $\mathfrak{h}^{\tau}$ a standard subalgebra. The standard subalgebras $\mathfrak{h}^{\tau}$ for inner automorphisms $\tau=\operatorname{Ad}(t)$ are in bijection with proper subdiagrams of the affine diagram of type $E_{6}$; these subalgebras all contain $\mathfrak{t}$ as a Cartan subalgebra. The standard subalgebras $\mathfrak{h}^{\tau}$ for outer automorphisms $\tau=\operatorname{Ad}(t) \vartheta$ are in bijection with proper subdiagrams of the affine diagram of type ${ }^{2} E_{6}$; these subalgebras all contain $\mathfrak{t}^{\vartheta}$ as a Cartan subalgebra.

The automorphisms $\theta$ and $\tau$ commute, so $\theta$ acts on the standard subalgebra $\mathfrak{k}:=\mathfrak{g}^{\tau}$. If $\tau$ is inner and $\vartheta$ acts nontrivially on the subdiagram for $\mathfrak{k}$ then $\left.\theta\right|_{\mathfrak{k}}$ is outer, because $\theta$ permutes a basis of the root-system of $\mathfrak{t}$ in $\mathfrak{k}$. And if $\tau$ is outer then $\left.\theta\right|_{\mathfrak{k}}$ must be inner, because $\theta$ acts trivially on the Cartan subalgebra $\mathfrak{t}^{\vartheta}$ of $\mathfrak{k}$. 
Suppose now that $\operatorname{rank}\left(\left.\theta\right|_{\mathfrak{k}}\right)=\operatorname{rank}(\theta)$, so that there is a Cartan subspace $\mathfrak{c}$ for $\theta$ such that $\mathfrak{c} \subset \mathfrak{k}$. Let $K=\operatorname{Aut}(\mathfrak{k})^{\circ}$ and let $\widetilde{K}$ be the connected subgroup of $H$ corresponding to $\mathfrak{k}$. These groups are normalized by $\theta$ and the natural map $\widetilde{K} \rightarrow K$ restricts to a surjection

$$
\widetilde{K}_{0}:=\left(\widetilde{K}^{\theta}\right)^{\circ} \longrightarrow\left(K^{\theta}\right)^{\circ}=: K_{0}
$$

which induces an isomorphism

$$
N_{\widetilde{K}_{0}}(\mathfrak{c}) / Z_{\widetilde{K}_{0}}(\mathfrak{c}) \simeq N_{K_{0}}(\mathfrak{c}) / Z_{K_{0}}(\mathfrak{c})
$$

It follows that we have an embedding of little Weyl groups

$$
W_{K}\left(\mathfrak{c},\left.\theta\right|_{\mathfrak{k}}\right) \hookrightarrow W_{H}(\mathfrak{c}, \theta)
$$

With the exception of number $2_{c}$, the next-to-right-most column of Table 23 below gives the Kac diagram of an $H$-conjugate $\theta^{\prime}$ of $\theta$ such that the subdiagram of $1^{\prime} s$ determines a standard subalgebra $\mathfrak{k}$ (given in the last column) such that

$$
\operatorname{rank}\left(\left.\theta\right|_{\mathfrak{k}}\right)=\operatorname{rank}(\theta) \quad \text { and } \quad W_{K}\left(\mathfrak{c},\left.\theta\right|_{\mathfrak{k}}\right)=W_{H}(\mathfrak{c}, \theta)
$$

and such that $\left.\theta\right|_{\mathfrak{k}}$ satisfies the conditions of Lemma 7.4. From [19, Prop. 5.2] it follows that $\theta$ admits a Kostant section contained in $\mathfrak{k}$.

In the table below we indicate $\mathfrak{k}=\mathfrak{h}^{\tau}$ as the subdiagram of $1^{\prime} s$ in a Kac diagram of type $E_{6}$ or ${ }^{2} E_{6}$ according to whether $\tau$ is inner or outer. Recall that $\left.\theta\right|_{\mathfrak{k}}$ is then outer or inner, respectively. The superscript ${ }^{2} X$ means that $\left.\theta\right|_{\mathfrak{k}}$ is outer. The notation ${ }^{2}\left(2 A_{2}\right)$ indicates that $\mathfrak{k} \simeq \mathfrak{s l}_{3} \oplus \mathfrak{s l}_{3}$ and $\theta$ swaps the two factors.

In the exceptional case $2_{c}$, previous work on involutions [16, Prop. 23] (for $k=\mathbb{C}$ ) and [18, 6.3] (for $p \neq 2$ ) shows that there is a $\theta$-stable subalgebra $\mathfrak{k} \simeq \mathfrak{s l}_{3}$ containing $\mathfrak{c}$ as a Cartan subalgebra, and $W_{H}(\mathfrak{c}, \theta)$ is just the ordinary Weyl group of $\mathfrak{c}$ in $\mathfrak{k}$. In this case $\theta$ is the unique (up to conjugacy) pinned involution of $\mathfrak{s l}_{3}$, which is known to have a Kostant section. 
Table 23: The gradings of positive rank in type $E_{6}$ (outer case)

\begin{tabular}{cccccccc}
\hline No. & $\left.\theta\right|_{\mathfrak{h}}$ & $w \in-W\left(E_{6}\right)$ & $w \in W\left(E_{7}\right)$ & $W_{H}\left(\mathfrak{c},\left.\theta\right|_{\mathfrak{h}}\right)$ & degrees & $\left.\theta^{\prime}\right|_{\mathfrak{h}}$ & $\mathfrak{k}$ \\
\hline $18_{a}$ & $111 \Leftarrow 11$ & $-E_{6}\left(a_{1}\right)$ & $E_{7}$ & $\boldsymbol{\mu}_{9}$ & 9 & $111 \Leftarrow 11$ & ${ }^{2} E_{6}$ \\
$12_{b}$ & $110 \Leftarrow 11$ & $-E_{6}$ & $E_{7}\left(a_{2}\right)$ & $\boldsymbol{\mu}_{12}$ & 12 & $-211 \Leftarrow 11$ & ${ }^{2} E_{6}$ \\
$10_{b}$ & $110 \Leftarrow 10$ & $-\left(A_{4}+A_{1}\right)$ & $D_{6}$ & $\boldsymbol{\mu}_{5}$ & 5 & $-311 \Leftarrow 11$ & ${ }^{2} E_{6}$ \\
$10_{a}$ & $101 \Leftarrow 01$ & $-\left(A_{4}+A_{1}\right)$ & $D_{6}$ & $\boldsymbol{\mu}_{5}$ & 5 & $-111 \Leftarrow 1-1$ & ${ }^{2} A_{5}$ \\
$10_{c}$ & $010 \Leftarrow 11$ & $-\left(A_{4}+A_{1}\right)$ & $D_{6}$ & $\boldsymbol{\mu}_{5}$ & 5 & $9-51 \Leftarrow 11$ & ${ }^{2} D_{5}$ \\
$8_{f}$ & $100 \Leftarrow 11$ & $-D_{5}$ & $D_{5}+A_{1}$ & $\boldsymbol{\mu}_{8}$ & 8 & $-411 \Leftarrow 11$ & ${ }^{2} E_{6}$ \\
$8_{c}$ & $010 \Leftarrow 10$ & $-D_{5}$ & $D_{5}+A_{1}$ & $\boldsymbol{\mu}_{8}$ & 8 & $111 \Leftarrow 1-4$ & $C_{4}$ \\
$6_{a}$ & $100 \Leftarrow 10$ & $-\left(3 A_{2}\right)$ & $E_{7}\left(a_{4}\right)$ & $G_{25}$ & $6,9,12$ & $-511 \Leftarrow 11$ & ${ }^{2} E_{6}$ \\
$6_{c}$ & $010 \Leftarrow 01$ & $-\left(2 A_{2}\right)$ & $D_{6}\left(a_{2}\right)+A_{1}$ & $G(3,1,2)$ & 3,6 & $211 \Leftarrow 1-6$ & ${ }^{2} A_{5}$ \\
$6_{g}$ & $000 \Leftarrow 11$ & $-A_{2}$ & $D_{4}+3 A_{1}$ & $\boldsymbol{\mu}_{6}$ & 6 & $-301 \Leftarrow 11$ & $B_{3}$ \\
$6_{i}$ & $001 \Leftarrow 00$ & $-\left(A_{5}+A_{1}\right)$ & $A_{5}^{\prime}$ & $\boldsymbol{\mu}_{3}$ & 3 & $011 \Leftarrow 0-2$ & ${ }^{2}\left(2 A_{2}\right)$ \\
$6_{k}$ & $110 \Leftarrow 00$ & $-\left(A_{5}+A_{1}\right)$ & $A_{5}^{\prime}$ & $\boldsymbol{\mu}_{3}$ & 3 & $011 \Leftarrow 2-6$ & ${ }^{2}\left(2 A_{2}\right)$ \\
$4_{b}$ & $000 \Leftarrow 10$ & $-D_{4}\left(a_{1}\right)$ & $2 A_{3}+A_{1}$ & $G_{8}$ & 8,12 & $-611 \Leftarrow 11$ & ${ }^{2} E_{6}$ \\
$4_{d}$ & $010 \Leftarrow 00$ & $-\left(A_{3}+2 A_{1}\right)$ & $\left(A_{3}+A_{1}\right)^{\prime \prime}$ & $\boldsymbol{\mu}_{4}$ & 4 & $111 \Leftarrow-20$ & $A_{3}$ \\
$4_{e}$ & $100 \Leftarrow 01$ & $-\left(A_{3}+2 A_{1}\right)$ & $\left(A_{3}+A_{1}\right)^{\prime \prime}$ & $\boldsymbol{\mu}_{4}$ & 4 & $-1-11 \Leftarrow 10$ & $B_{2}$ \\
$2_{a}$ & $000 \Leftarrow 01$ & -1 & $7 A_{1}$ & $W\left(E_{6}\right)$ & $2,5,6,8,9,12$ & $-711 \Leftarrow 11$ & ${ }^{2} E_{6}$ \\
$2_{c}$ & $100 \Leftarrow 00$ & $-\left(4 A_{1}\right)$ & $\left(3 A_{1}\right)^{\prime}$ & $W\left(A_{2}\right)$ & 2,3 & --- & ${ }^{2} A_{2}$ \\
\hline & & & & & \\
\end{tabular}

\section{References}

[1] M. Bhargava, A. Shankar, Binary quartic forms having bounded invariants, and the boundedness of the average rank of elliptic curves, ArXiv: 1006.1002 (2010).

[2] __ Ternary cubic forms having bounded invariants and the existence of a positive proportion of elliptic curves having rank 0, ArXiv: 1007.0052 (2010).

[3] A. Borel, Properties and linear representations of Chevalley groups, Seminar in Algebraic Groups and related finite groups, Lecture Notes in Math. 131, Springer-Verlag (1970), pp. 1-55.

[4] _ Automorphic L-functions, Proc. Symp. Pure Math., vol. 33, part 2, Amer. Math. Soc., Providence, RI (1979), pp. 27-61.

[5] _ Linear algebraic groups, Graduate Texts in Mathematics, vol. 126, Springer-Verlag New York, 1991.

[6] N. Bourbaki, Lie groups and Lie algebras, Chap. 4-6, Springer-Verlag, Berlin, 2002.

[7] R. Carter, Conjugacy classes in the Weyl group, Compositio Math., 25 (1972), pp. 1-59. 
[8] W. de Graaf, Computing representatives of nilpotent orbits of $\theta$-groups, J. Symbolic Comput., 46 (2011), pp. 438-458.

[9] W. de Graaf, O. Yakimova, Good index behaviour of $\theta$-representations, Algebr. Represent. Theory, to appear.

[10] B. Gross, On Bhargava's representations and Vinberg's invariant theory, preprint 2011.

[11] B. Gross, M. Reeder, Arithmetic invariants of discrete Langlands parameters, Duke Math. Jour., 154 (2010), pp. 431-508.

[12] V. Kac Infinite dimensional Lie algebras, 3rd ed., Cambridge, 1995.

[13] D. Kazhdan and G. Lusztig, Fixed point varieties on affine flag manifolds, Israel Jour. Math., 62 (1988), pp. 129-168.

[14] B. Kostant, The principal three-dimensional subgroup and the Betti numbers of a complex simple Lie group, Amer. J. Math., 81 (1959), pp. 973-1032.

[15] _ Groups over $\mathbb{Z}$, Proc. Symp. Pure Math., vol. 9, Amer. Math. Soc., Providence, RI (1966), pp. 71-83.

[16] B. Kostant, S. Rallis, Orbits and representations associated with symmetric spaces, Amer. J. Math., 93 (1971), pp. 753-809.

[17] S. Kumar, G. Lusztig, D. Prasad, Characters of simplylaced nonconnected groups verus characters of nonsimplylaced connected groups, Contemp. Math., 478 (2009), pp. 99-101.

[18] P. Levy, Involutions of reductive Lie algebras in positive characteristic, Adv. Math., 210, no. 2, (2007), pp. 505-559.

[19] _ Vinberg's $\theta$-groups in positive characteristic and Kostant-Weierstrass slices, Transform. Groups, 14, no. 2, (2009), pp. 417-461.

[20] _ $K W$-sections for exceptional type Vinberg's $\theta$-groups, preprint.

[21] I.G. Macdonald, Affine Hecke Algebras and Orthogonal Polynomials, Camb. Univ. Press 2005.

[22] D. Mumford, Stability of projective varieties, L'Enseignement Math., 23(1) (1977), pp. 39-110.

[23] D. Panyushev, On invariant theory of -groups, Jour. Algebra, 283 (2005), pp. 655-670.

[24] M. Reeder, Torsion automorphisms of simple Lie algebras, L'Enseignement Math., 56(2) (2010), pp. 3-47.

[25] _ Elliptic centralizers in Weyl groups and their coinvariant representations, Representation Theory, 15 (2011), pp. 63-111.

[26] J.-P. Serre, Coordonnées de Kac, Oberwolfach Reports, 3 (2006), pp. 1787-1790.

[27] G.C. Shephard, J.A. Todd Finite unitary reflection groups, Canadian J. Math., 6 (1954), pp. 274304. 
[28] N. Spaltenstein, On the Kazhdan-Lusztig map for exceptional Lie algebras, Adv. Math., 83 (1990), pp. 48-74.

[29] T.A. Springer, Regular elements in finite reflection groups, Inv. Math., 25 (1974), pp. 159-198.

[30] R. Steinberg, Variations on a theme of Chevalley, Pacific J. Math. 9 no.3 (1959), pp. 875-891.

[31] , Regular elements of semisimple algebraic groups, Publ. Math. Inst. Hautes Études Sci. 25 (1965), pp. 49-80.

[32] __ Lectures on Chevalley groups, Yale Lecture Notes, 1967.

[33] _ Endomorphisms of linear algebraic groups, Mem. Amer. Math. Soc., 80 (1968).

[34] _ Torsion in reductive groups, Advances in Math. 15 (1975), pp. 63-92.

[35] E.B. Vinberg, The Weyl group of a graded Lie algebra, Izv. Akad. Nauk SSSR Ser. Mat., 40 no. 3 (1976), pp. 488-526. English translation: Math. USSR-Izv. 10 (1977), pp. 463-495.

[36] W.C. Waterhouse Introduction to affine group schemes, Springer-Verlag, New York, 1979. 